\title{
Min-Max Results in Combinatorial Optimization
}

\author{
A. Schrijver \\ Universiteit van Amsterdam, Instituut voor Actuariaat en Econometrie, \\ Jodenbreestraat 23, $1011 \mathrm{NH}$ Amsterdam, The Netherlands
}

\section{Introduction}

Often the optimum of a combinatorial optimization problem is characterized by a min-max relation, asserting that the maximum value in one combinatorial optimization problem is equal to the minimum value in some other optimization problem. One of the best-known examples is the max-flow min-cut theorem of Ford and Fulkerson [1956] and Elias, Feinstein and Shannon [1956]: If "nodes" $1, \ldots, n$ and "capacities" $c_{i j} \geqslant 0(i, j=1, \ldots, n)$ are given, then the maximum value of a flow from "source node" 1 to "sink node" $n$, subject to $c$, is equal to the minimum capacity of a cut separating 1 from $n$, i. e., to

$$
\min \sum_{i \in T} \sum_{j \notin T} c_{i, j}
$$

where the minimum ranges over all subsets $T$ of $\{1, \ldots, n\}$ with $1 \in T$ and $n \notin T$. Moreover, if the capacities are integer there exists an integer optimum flow. Here a flow from 1 to $n$ subject to $c$ is a vector $\left(x_{i j}\right)_{i, j=1, \ldots, n}$ satisfying

$$
\begin{array}{ll}
\text { (i) } \sum_{i=1}^{n} x_{i j}=\sum_{i=1}^{n} x_{j i} & (j=2, \ldots, n-1), \\
\text { (ii) } 0 \leqslant x_{i j} \leqslant c_{i j} & (i, j=1, \ldots, n) .
\end{array}
$$

The value of the flow is the "net amount" of flow leaving node 1 , which is

$$
\sum_{i=1}^{n} x_{1 i}-\sum_{i=1}^{n} x_{i 1}
$$

(This is clearly equal to the net amount of flow entering node $n$.)

Generally, a combinatorial min-max relation is closely related to the algorithmic solvability of the corresponding optimization problem. Often a minmax relation appears as a by-product of an algorithm, and it can serve as an optimality criterion, and as a "good characterization", for the optimization problem. In turn, with the ellipsoid method a min-max relation sometimes gives that the combinatorial optimization problem can be solved within time bounded by a polynomial in the problem size. Moreover, a min-max relation can be used in a sensitivity analysis, giving insight in the question in how much the optimum changes if we vary the initial constraints.

Besides, combinatorial min-max relations are of theoretical interest. Usually, they yield elegant combinatorial theorems, and they allow a geometrical 
representation of the problems in terms of polyhedra. Such theoretical considerations have turned out to be very useful in understanding the algorithmic practice.

Historically, the first combinatorial min-max relations were found by König [1916, 1932], Menger [1927] and Tutte [1947]. They deal with maximum matchings and path packings, and at first they were formulated and proved purely combinatorially. The algorithmic and polyhedral aspects of combinatorial min-max relations were revealed in the 1950 s by the work of Ford, Fulkerson and Hoffman on bipartite matchings and network flows, and were founded and developed further in the 1960s by the pioneering results of Edmonds on matchings, matroids and branchings. Further significant min-max results were discovered in the 1970s by Lovász (perfect graphs, matroid matching), Seymour (binary hypergraphs), Lucchesi and Younger (directed cuts), Mader (S-paths).

We now first go further into the polyhedral and algorithmic aspects of combinatorial min-max relations. For a more comprehensive review of "polyhedral combinatorics", see W. R. Pulleyblank's survey.

Polyhedral aspects of min-max relations. Often a combinatorial min-max relation amounts to the fact that a certain linear program has an integer optimal (primal and/or dual) solution, without requiring integrality explicitly in advance. The duality theorem of linear programming then gives the min-max relation.

E.g., the max-flow min-cut theorem is equivalent to the fact that the linear program of maximizing (3) subject to (2) has an integer optimal dual solution. Moreover, if $c$ is integer, the linear program has an integer optimal primal solution.

In this example the integrality, and hence the combinatorial min-max relation, follows from the total unimodularity of (2). Total unimodularity is one important tool in integer linear programming and combinatorial optimization, due to Hoffman and Kruskal. For most of the combinatorial min-max relations, however, the reduction to LP-duality requires more than just total unimodularity. Consider, e.g., the following problem.

Let nodes $1, \ldots, n$, together with (possibly asymmetric) "lengths" $l_{i j} \geqslant 0$ $(i, j=1, \ldots, n)$ be given. Suppose we wish to select certain directed trajects between the nodes such that each node $j \neq 1$ is reachable from node 1 by a directed path, and such that the total length of the selected trajects is as small as possible. That is, we wish to find a shortest 1-arborescence. It is easy to see that this amounts to the following integer linear programming problem:

$$
\begin{array}{ll}
\min \sum_{i, j=1}^{n} l_{i j} x_{i j} & \\
\text { subject to } & \\
\sum_{j \in T} \sum_{i \notin T} x_{i j} \geqslant 1 & (T \subseteq\{2, \ldots, n\}, T \neq \emptyset), \\
\quad x_{i j} \geqslant 0 & (i, j=1, \ldots, n), \\
x_{i j} \text { integer } & (i, j=1, \ldots, n) .
\end{array}
$$


Now it is a theorem of Edmonds [1967 a] that this minimum is not decreased if we delete the integrality condition. So the optimum value in (4) is equal to the common value in the linear programming duality equation:

$$
\begin{array}{ccc}
\min \sum_{i, j} l_{i j} x_{i j} & =\max \sum_{T} y_{T} \\
\text { subject to } & \text { subject to } & \\
\sum_{j \in T} \sum_{i \notin T} x_{i j} \geqslant 1 & (\forall T), \quad \sum_{T \nexists i, T \ni j} y_{T} \leqslant l_{i j} & (\forall i, j), \\
x_{i j} \geqslant 0 & (\forall i, j), \quad y_{T} \geqslant 0 & (\forall T),
\end{array}
$$

where $i$ and $j$ range over $1, \ldots, n$, and $T$ ranges over all nonempty subsets of $\{2, \ldots, n\}$.

So we now have two equivalent formulations of Edmonds' result: as the assertion that the minimum in (5) has an integer optimum solution, or as a minmax relation equating the minimum in (4) to the maximum in (5). There is a third equivalent interpretation: the vertices of the polyhedron defined by the constraints for the minimum in (5) are integer (and hence the incidence vectors of 1-arborescences). This of course follows from the fact that the minimum in (5) has an integer optimum solution $x_{i j}$ for each length function $l_{i j} \geqslant 0$. So Edmonds' min-max relation can be stated alternatively as a polyhedral result.

We emphasize here that this combinatorial min-max relation is not just reduced to LP-duality: the point is to prove the existence of integer optimum solutions. (Fulkerson [1974] extended Edmonds' result by showing that also the maximum in (5) has an integer optimum solution, if the lengths are integer.)

Algorithmic aspects of min-max relations. First, a min-max relation often follows as a by-product of an algorithm to solve the optimization problem. Typically the process is as follows. First one easily shows the inequality $\max \leqslant \min$. Next an algorithm for, say, the maximization problem has as main step a procedure which either improves the current solution, or shows that this solution is optimum by constructing a feasible solution for the minimization problem with the same objective value as that of the current solution.

Thus the relation $\max \leqslant \min$ proves the optimality of the algorithm, while, in turn, the algorithm yields the inequality $\max \geqslant \min$.

E. g., Ford and Fulkerson's maximum flow algorithm hangs on the subroutine which either finds an "augmenting path" improving the current flow, or finds a cut separating source and sink with capacity equal to the value of the current flow. So the max-flow min-cut theorem follows. Note that Ford and Fulkerson's algorithm is at the same time a minimum cut algorithm.

Similarly, Edmonds' min-max formula for minimum length 1-arborescences given above follows as a by-product of an algorithm finding a shortest 1-arborescence.

The above also shows the role of a min-max relation as an optimality criterion. E. g., in the case of max-flow min-cut, a given flow can be shown to be optimum by specifying a cut separating source and sink, with capacity equal to the value of the given flow. 
Frequently (in fact, in all cases treated in this paper) a min-max relation yields a good characterization. This means that the problem has an answer, the correctness of which can be shown by a proof of length bounded by a polynomial in the size of the problem instance. (This concept of good characterization is due to Edmonds [1965 a].)

Indeed, suppose we have a min-max relation for a combinatorial optimization problem. To give a good characterization it suffices to display a feasible solution for the maximization problem, together with a feasible solution for the minimization problem, with the same objective value. If such feasible solutions exist of polynomially bounded size (which usually is the case), we have a polynomial-length proof of the optimality of the solution.

In "NP-language", a problem has a good characterization if and only if it

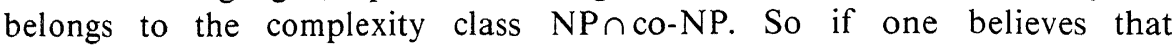
$N P \neq N P \cap$ co-NP, one may not expect a satisfactory min-max relation for any NP-complete problem - satisfactory in the sense of supplying a good characterization.

Always, a polynomial algorithm yields a good characterization (i.e., $\mathrm{P} \subseteq \mathrm{NP} \cap \mathrm{co}-\mathrm{NP})$ : one can prove any answer to be correct just by writing down the steps of the algorithm (which takes polynomial space), together with a proof of the correctness of the algorithm (which takes only fixed space, independent of the special instance of the problem).

The reverse implication, that a good characterization yields a polynomial algorithm, is not known. However, the combinatorial optimization problems investigated so far have created the impression that if a problem has a good characterization, it also is polynomially solvable. Recently, this impression has got the added support of the ellipsoid method, which sometimes deduces polynomial solvability from a min-max relation, or from the associated polyhedral representation (cf. Grötschel, Lovász and Schrijver [1981]).

To explain this, suppose a certain combinatorial optimization problem is accompanied by a min-max relation which amounts to the fact that the minimum in the LP-duality equation

$$
\min \{w x \mid A x \geqslant b\}=\max \{y b \mid y \geqslant 0, y A=w\}
$$

has an integer optimum solution $x$. Now, roughly speaking, if for each vector $x$, the system $A x \geqslant b$ can be checked in polynomial time, then with the ellipsoid method the minimum in (6) can be determined in polynomial time as well. So the polynomial solvability of the combinatorial optimization problem follows.

Here "checking" means testing whether $A x \geqslant b$ holds, and, if not, finding a violated inequality. If the size of the system $A x \geqslant b$ is bounded by a polynomial in the size of the original combinatorial optimization problem, we can check $A x \geqslant b$ by testing the constraints one by one (and (6) can be solved with Khachiyan's method for linear programming). However, it is not necessary that the number of inequalities in $A x \geqslant b$ is polynomially bounded. There may exist other ways of checking $A x \geqslant b$ than testing the constraints one by one.

Consider, e.g., the example of 1-arborescences given above. Finding a shortest 1-arborescence means solving (4), which is, by Edmonds' theorem, the 
same as solving (5). Now in the minimum of (5) there are exponentially many constraints. Yet these constraints can be checked in polynomial time. Given a vector $\left(x_{i j}\right)$, first test whether all components are nonnegative. This can be done easily in polynomial time. If one of the components is negative we have found a violated inequality. If they all are nonnegative, consider $x_{i j}$ as a capacity function. Next find for each $j \neq 1$ (e.g. with Ford and Fulkerson's minimum cut algorithm) a cut $C_{j}$ separating node 1 from node $j$, with minimum capacity. Taking the minimum of the capacities of $C_{2}, \ldots, C_{n}$ gives a nonempty subset $T$ of $\{2, \ldots, n\}$ minimizing $\sum_{j \in T, i \notin T} x_{i j}$. If this minimum is less than $1, T$ determines a violated inequality. Otherwise we may conclude that the constraints for the minimum in (5) hold for $\left(x_{i j}\right)$. So with ellipsoids also the minimum in (5), and hence a shortest 1-arborescence, can be found in polynomial time.

It must be admitted immediately that Edmonds' direct polynomial algorithm for the shortest 1-arborescence problem is much more efficient (and gives the polyhedral results as a by-product). With the ellipsoid method, however, the polynomial solvability can be shown quickly. Moreover, for some other combinatorial optimization problems the polynomial solvability could be derived, as yet, only by combining a min-max relation with the ellipsoid method.

Concluding, a min-max relation often follows from an algorithm, but can in turn be helpful in providing not only a good characterization, but sometimes even the polynomial solvability of the problem.

Finally we notice that the interpretation of a combinatorial min-max relation in terms of LP-duality also enables to extend the known sensitivity analysis for linear programming to a sensitivity analysis for the combinatorial optimization problem. Thus if we have solved both the maximization and the minimization problem, the optimum solutions give us some of the tight constraints.

E.g., in the max-flow min-cut problem each of the arcs in the minimum-capacitated cut has a tight capacity. If we tighten any of these capacities, the maximum flow value will decrease, whereas if we wish to increase the flow value we must relax at least one of these capacities.

Unfortunately, combinatorial optima considered as LP-optima often turn out to be highly degenerate, so that such a duality analysis provides not more than an upper bound for the new maximum, also marginally (lower bound for the new minimum, respectively). E. g., in the max-flow min-cut case there often exist several cuts of minimum capacity, and in each of these cuts we have to relax some capacity if we want to increase the flow value.

Below we give a survey of combinatorial min-max relations discovered so far, and we go into the methods for deriving them. Above we passed already on two types of methods: algorithmic methods, which consist of showing that the maximum value found by an algorithm is equal to the minimum value in some other problem, and polyhedral methods, which consist of showing that certain linear programs have integer optimum solutions. A third "type" of method could be named combinatorial, where purely combinatorial, mostly graph-theoretical techniques are used, generally without a direct algorithmic relevance.

We first give in Section 2 some preliminaries on terminology, notation and 
conventions. In Section 3 we briefly review the notions of total unimodularity and total dual integrality and the theory of blocking and anti-blocking polyhedra (for a more comprehensive survey, see Pulleyblank [1982]). In the Sections 4 and 5 we describe min-max relations for bipartite graphs and network flows, respectively, which turn out to be a basis, and a special case, of several of the min-max relations we discuss further. These are grouped around the subjects of (nonbipartite) matchings and coverings (Section 6), multicommodity flows (Section 7), arborescences and directed cuts (Section 8), perfect graphs (Section 9), clutters and blockers (Section 10), and matroids and submodular functions (Section 11).

Some references for background information are: for graph theory, Bondy and Murty [1976] or Wilson [1972]; for a survey of polynomial algorithms in combinatorial optimization, Lawler [1976]; for complexity theory (NP-completeness), Garey and Johnson [1979]; for both latter two subjects, Papadimitriou and Steiglitz [1982]; for polyhedral theory, Pulleyblank [1983] and Stoer and Witzgall [1970].

\section{Some Terminology, Notation and Conventions}

Throughout this paper we assume familiarity with the basic concepts from graph theory and linear programming.

An (un)directed graph is a pair $(V, E)$ where $V$ is a finite set (of vertices or points) and $E$ is a collection of (un)ordered pairs (called the edges in the undirected case, and arcs in the directed case). The set $E$ may contain a pair more than once, i. e., parallel edges or arcs are allowed.

Invited by the figurative character of graphs we shall often use loose language, which will shorten arguments, and which could be made as formal as we like. Thus we use expressions like: "add a new vertex", "replace an edge by two parallel edges", "replace an edge by a path of length three" (which means replace $\{u, v\}$ by $\left\{u, u^{\prime}\right\},\left\{u^{\prime}, v^{\prime}\right\},\left\{v^{\prime}, v\right\}$, where $u^{\prime}$ and $v^{\prime}$ are new vertices), etc.

A graph $G=(V, E)$ is bipartite if $V$ can be split into classes $V_{1}$ and $V_{2}$ such that every edge or arc of $G$ contains a vertex in $V_{1}$ and a vertex in $V_{2}$. We shall call $V_{1}$ and $V_{2}$ the colour classes of $G$, although this splitting need not be unique.

If $G=(V, E)$ is an undirected graph, and $V^{\prime} \subseteq V$, the subgraph induced by $V^{\prime}$, denoted by $\left\langle V^{\prime}\right\rangle$, is the graph $\left(V^{\prime},\left\{e \in E \mid e \subseteq V^{\prime}\right\}\right)$.

If $r$ and $s$ are vertices of an (un)directed graph, an $r$-s-path is an (un)directed path from $r$ to $s$. Sometimes, if no danger of confusion exists, we shall identify a subgraph or a path with the set of edges or arcs contained in it. Two subgraphs or paths are edge-disjoint (arc-disjoint, vertex-disjoint, respectively) if they have no edges (arcs, vertices, respectively) in common. Two paths are internally vertex-disjoint if they have no vertices in common, except possibly for the end points.

The complete undirected graph on $n$ vertices is denoted by $K_{n}$, and the complete undirected bipartite graph with colour classes of sizes $m$ and $n$, by $K_{m, n}$. 
If $G=(V, E)$ is an undirected graph and $V^{\prime} \subseteq V$, then $\delta\left(V^{\prime}\right)$ or $\delta_{E}\left(V^{\prime}\right)$ denotes the set of edges of $G$ with exactly one vertex in $V^{\prime}$. If $D=(V, A)$ is a directed graph and $V^{\prime} \subseteq V$, then $\delta^{+}\left(V^{\prime}\right)$ or $\delta_{A}^{+}\left(V^{\prime}\right)$ denotes the set of arcs leaving $V^{\prime}$. Similarly, $\delta^{-}\left(V^{\prime}\right)$ or $\delta_{A}^{-}\left(V^{\prime}\right)$ denotes the set of arcs entering $V^{\prime}$.

We often use just $v$ instead of $\{v\}$.

If $A$ is a matrix, and $w$ and $b$ are vectors, then three (equivalent) forms of the duality theorem of linear programming are:

$$
\begin{aligned}
& \max \{w x \mid x \geqslant 0, A x \leqslant b\}=\min \{y b \mid y \geqslant 0, y A \geqslant w\}, \\
& \max \{w x \mid A x \leqslant b\} \quad=\min \{y b \mid y \geqslant 0, y A=w\}, \\
& \min \{w x \mid A x \geqslant b\} \quad=\max \{y b \mid y \geqslant 0, y A=w\} .
\end{aligned}
$$

Here any of these relations holds if at least one of the two optima is finite.

In (1), and in similar expressions throughout this paper, we use the following conventions. Concatenations $w x$ and $y b$ denote inner products. If $v$ and $w$ are vectors, $v \leqslant w$ denotes component-wise comparison. When using expressions like $w x, A x \leqslant b, y A \geqslant w$ we implicitly assume compatibility of sizes. Moreover, 0 and 1 denote (also) the all-zero and the all-one vectors, of appropriate length.

For a vector $w,|w|$ denotes the sum of its components (so $|w|=w 1$ ).

$\mathbb{R}, \mathbb{Q}, \mathbb{Z}, \frac{1}{2} \mathbb{Z}$ denote the sets of reals, rationals, integers, half-integers, respectively, while the subscript + restricts such sets to their nonnegative elements.

Let $S$ be a finite set. We identify functions $x: S \rightarrow \mathbb{R}$ with vectors $x$ in $\mathbb{R}^{S}$. Furthermore, we identify any subset $S^{\prime}$ of $S$ with its incidence vector $\chi_{S^{\prime}}$ in $\mathbb{R}^{S}$, defined by

$$
\chi_{S^{\prime}}(s)=1 \text {, if } s \in S^{\prime} \text {, and } \chi_{S^{\prime}}(s)=0 \text {, if } s \notin S^{\prime},
$$

for $s$ in $S$. Hence any collection $\mathscr{C}$ of subsets of $S$ is also a collection of vectors in $\mathbb{R}^{S}$, and "the convex hull of (the elements of) $\mathscr{C}$ " means the convex hull of the incidence vectors of the elements of $\mathscr{C}$. E. g., the convex hull of the matchings is the convex hull of the incidence vectors of the matchings.

If $c: S \rightarrow \mathbb{R}$, then we denote

$$
c\left(S^{\prime}\right):=\sum_{s \in S^{\prime}} c(s),
$$

for $S^{\prime} \subseteq S$. If $c$ is called a "capacity" ("length", "weight", ...) function, then $c\left(S^{\prime}\right)$ is called the capacity (length, weight, ...) of $S^{\prime}$.

Further nonstandard notation and terminology used below will be defined on the spot.

\section{Total Unimodularity, Total Dual Integrality, and Blocking and Anti-Blocking Polyhedra}

The concepts mentioned in the title of this section play an important role in the field of polyhedral methods for combinatorial min-max relations. Here we re- 
view these notions in brief. For a more extensive treatment, see the survey on "polyhedral combinatorics" by W. R. Pulleyblank.

Total unimodularity. A matrix $A$ is called totally unimodular if each square submatrix of $A$ has determinant $0,+1$ or -1 . In particular, each entry of $A$ is $0,+1$ or -1 .

The interest of totally unimodular matrices for optimization was discovered by the following theorem of Hoffman and Kruskal [1956]: if $A$ is totally unimodular and $b$ and $w$ are integer vectors, then both sides of the LP-duality equation

$$
\max \{w x \mid A x \leqslant b\}=\min \{y b \mid y \geqslant 0, y A=w\}
$$

have integer optimum solutions.

It follows that any linear program with totally unimodular constraint matrix and integer right hand sides has an integer optimum primal solution.

If $A$ is totally unimodular, then also, e.g., the matrices $[-I A]$ and $[I-I A-A]$ are totally unimodular. Hence also each of the optima in

$$
\max \{w x \mid x \geqslant 0, A x \leqslant b\}=\min \{y b \mid y \geqslant 0, y A \geqslant w\}
$$

and in

$$
\begin{aligned}
& \max \left\{w x \mid c_{1} \leqslant x \leqslant c_{2}, b_{1} \leqslant A x \leqslant b_{2}\right\}= \\
& \min \left\{y_{2} b_{2}-y_{1} b_{1}+z_{2} c_{2}-z_{1} c_{1} \mid\left(y_{2}-y_{1}\right) A+z_{2}-z_{1}=w\right\}
\end{aligned}
$$

has an integer optimum solution, for integer $b, w, b_{1}, b_{2}, c_{1}, c_{2}$. In fact, Hoffman and Kruskal proved that a matrix $A$ is totally unimodular if and only if it in integer and the maximum in (2) has an integer optimum solution $x$ for all integer vectors $b$ and $w$ for which this maximum exists. That is, if and only if the polyhedron $\{x \mid x \geqslant 0, A x \leqslant b\}$ has integer vertices, for each integer vector $b$.

Ghouila-Houri [1962] gave the following characterization of total unimodularity: a matrix $A$ is totally unimodular, if and only if each collection $R$ of rows of $A$ can be split into classes $R_{1}$ and $R_{2}$ such that the sum of the rows in class $R_{1}$ minus the sum of the rows in class $R_{2}$ is a vector with components $0,+1,-1$ only.

Total dual integrality. A second useful concept, introduced by Edmonds and Giles [1977], is defined as follows. A system $A x \leqslant b$ of linear inequalities is called totally dual integral if the minimum in the LP-duality equation

$$
\max \{w x \mid A x \leqslant b\}=\min \{y b \mid y \geqslant 0, y A=w\}
$$

has an integer optimum solution $y$, for each integer objective function $w$ for which the optima exist.

Motivation for this concept comes from the following result of Edmonds and Giles: if $A x \leqslant b$ is totally dual integral and the right hand side $b$ is integer, then the maximum in (4) has an integer optimum solution for each objective function $w$ for which the optima exist. That is, each face of the polyhedron $\{x \mid A x \leqslant b\}$ contains integer vectors. 
So if $A x \leqslant b$ is totally dual integral and if for some $w$ the optima (4) exist, then the minimum in (4) has an integer optimum solution if $w$ is integer, and the maximum in (4) has an integer optimum solution if $b$ is integer.

Total dual integrality forms a useful proof technique, as showing the existence of integer optimum dual solutions in (4) suffices for showing integer optimum primal and dual solutions.

Several systems of linear inequalities have been shown to be totally dual integral, and below we shall meet a number of them.

A system $A x \leqslant b$ is called totally dual half-integral if the minimum in (4) has a half-integer optimum solution $y$ for each integer vector $w$ for which the minimum exists.

Blocking and anti-blocking polyhedra. Fulkerson [1970, 1971, 1972] and Lehman [1979] developed a theory of "blocking" and "anti-blocking" polyhedra, which throws a new light on combinatorial min-max relations through the classical polarity of vertices and facets of polyhedra.

Let $A$ and $B$ be nonnegative matrices, both with $n$ columns, and with rows $a_{1}, \ldots, a_{t}$ and $b_{1}, \ldots, b_{m}$, respectively. Suppose we have the following relation between $A$ and $B$ :

$$
\left\{x \in \mathbb{R}_{+}^{n} \mid B x \geqslant 1\right\}=\left\{x \in \mathbb{R}_{+}^{n} \mid x \geqslant y\right.
$$

for some convex combination $y$ of $\left.a_{1}, \ldots, a_{t}\right\}$.

Then the same holds if we interchange $A$ and $B$ :

$$
\begin{aligned}
& \left\{x \in \mathbb{R}_{+}^{n} \mid A x \geqslant 1\right\}=\left\{x \in \mathbb{R}_{+}^{n} \mid x \geqslant y\right. \\
& \text { for some convex combination } \left.y \text { of } b_{1}, \ldots, b_{m}\right\} .
\end{aligned}
$$

This duality principle (which can be shown easily, e.g., with Farkas' lemma or with the LP-duality theorem) can be formulated equivalently as follows. Suppose that

$$
\min \left\{w a_{1}, \ldots, w a_{t}\right\}=\max \{|y| \mid y \geqslant 0, y B \leqslant w\}
$$

for each $w$ in $\mathbb{R}_{+}^{n}$. Then also

$$
\min \left\{w b_{1}, \ldots, w b_{m}\right\}=\max \{|y| \mid y \geqslant 0, y A \leqslant w\}
$$

for each $w$ in $\mathbb{R}_{+}^{n}$. Indeed, (7) is equivalent to (5) (by applying LP-duality to the maximum in (7)), and similarly (8) is equivalent to (6).

So if we have proved one min-max relation, viz. (7), we get another minmax relation, viz. (8), as a present. And conversely.

Fulkerson gave several interesting combinatorial applications of this equivalence, especially if $A$ and $B$ are $\{0,1\}$-matrices. He defined for any polyhedron $P \subseteq \mathbb{R}^{n}$ the blocking polyhedron $b(P)$ of $P$ by

$$
b(P):=\left\{z \in \mathbb{R}_{+}^{n} \mid z x \geqslant 1 \text { for all } x \text { in } P\right\},
$$

and observed that the polyhedron (6) is the blocking polyhedron of (5), and vice versa. 
Similarly a theory of anti-blocking polyhedra was obtained, where $\geqslant$ in (5), (6) and (9) is replaced by $\leqslant$.

\section{Bipartite Graphs}

We start with surveying min-max relations for bipartite graphs, as they form non-trivial special cases of many other combinatorial min-max relations, and as they exhibit several typical min-max phenomena.

Min-max relations for bipartite graphs are closely related, and for a part equivalent, to those for network flows to be treated in the next section.

First we give some standard notation and terminology. Let $G=(V, E)$ be an undirected graph. Then

(1) $v(G)=$ the matching number of $G=$ the maximum size of a matching in $G$ [a matching is a set of pairwise disjoint edges];

$\tau(G)=$ the vertex-cover number of $G=$ the minimum size of a vertex-cover for $G$ [a vertex-cover is a set of vertices intersecting every edge];

$\alpha(G)=$ the coclique number of $G=$ the maximum size of a coclique in $G$ [a coclique is a set of pairwise non-adjacent vertices];

$\rho(G)=$ the edge-cover number of $G=$ the minimum size of an edge-cover for $G$ [an edge-cover is a set of edges covering $V$ ].

It is easy to see that the inequalities $v(G) \leqslant \tau(G)$ and $\alpha(G) \leqslant \rho(G)$ always hold. The triangle $K_{3}$ shows that generally we do not have equality. In fact, equality in one of these relations implies equality in the other, as Gallai [1958, 1959] proved the following.

Theorem 1 (Gallai's theorem). For any undirected graph $G=(V, E)$ one has

$$
\alpha(G)+\tau(G)=|V|=v(G)+\rho(G)
$$

(where the second equality holds if $G$ has no isolated vertices).

Proof. (i) A set of vertices is a coclique, if and only if its complement is a vertex-cover. This proves $\alpha(G)+\tau(G)=|V|$.

(ii) We next show that $v(G)+\rho(G) \leqslant|V|$. Let $E^{\prime}$ be a collection of $v(G)$ pairwise disjoint edges. Hence $E^{\prime}$ covers $2 v(G)$ vertices. Choose for any vertex not covered by $E^{\prime}$ an arbitrary edge containing that vertex, and let $E^{\prime \prime}$ be the set of these edges. So $\left|E^{\prime \prime}\right|=|V|-2 v(G)$, and $E^{\prime} \cup E^{\prime \prime}$ covers all vertices. Therefore

$$
\rho(G) \leqslant\left|E^{\prime} \cup E^{\prime \prime}\right|=v(G)+|V|-2 v(G)=|V|-v(G) .
$$

(iii) To show that $v(G)+\rho(G) \geqslant|V|$, let $E^{\prime}$ be a collection of $\rho(G)$ edges covering $V$. Then the graph $\left(V, E^{\prime}\right)$ has no paths of lenght three (otherwise we could delete the middle edge to obtain a smaller edge-cover). Hence each component of $\left(V, E^{\prime}\right)$ is a "star". It is easy to see that there are $|V|-\rho(G)$ such stars. Choosing from each component one edge gives us $|V|-\rho(G)$ pairwise disjoint edges. Hence $v(G) \geqslant|V|-\rho(G)$. 
Deming [1979], Sterboul [1979] and Lovász [1982] characterized the undirected graphs $G$ with $v(G)=\tau(G)$ and $\alpha(G)=\rho(G)$. These graphs include the bipartite graphs, by the following theorem, which was presented in König [1931], but which finds its roots in earlier papers by Frobenius [1912, 1917] and König [1915, 1916].

Theorem 2 (König's matching theorem). The maximum size of a matching in a bipartite graph is equal to the minimum number of vertices intersecting all edges, i.e., $v(G)=\tau(G)$ for bipartite graphs $G$.

Proof. Let a matching $M$ in the bipartite graph $G=(V, E)$ be given. We describe a procedure which either finds a larger matching, or finds a vertex-cover $V^{\prime}$ with $\left|V^{\prime}\right|=|M|$. Obviously this proves the theorem.

Let $G$ have colour classes $V_{1}$ and $V_{2}$. Orient the edges in $M$ from $V_{2}$ to $V_{1}$, and orient all other edges of $G$ from $V_{1}$ to $V_{2}$. Let $V_{0}$ be the set of vertices covered by M. Now there are two possibilities.

(i) There is a directed path from $V_{1} \backslash V_{0}$ to $V_{2} \backslash V_{0}$. Necessarily, the second, fourth, sixth, ... arc in this path belongs to $M$, and the other not. Since the set $P$ of edges occurring in this path has odd cardinality, the symmetric difference $M \triangle P$ is a matching larger than $M$.

(ii) There is no directed path from $V_{1} \backslash V_{0}$ to $V_{2} \backslash V_{0}$. Let

$$
\begin{aligned}
V^{\prime}:= & \left\{v \in V_{1} \mid \text { there is no directed path from } V_{1} \backslash V_{0} \text { to } v\right\} \cup \\
& \left\{v \in V_{2} \mid \text { there is a directed path from } V_{1} \backslash V_{0} \text { to } v\right\} .
\end{aligned}
$$

It is not difficult to see that $V^{\prime}$ is a vertex-cover, and that $\left|V^{\prime}\right|=|M|$.

This proof, due to Ford and Fulkerson [1956], is an example of an algorithmic proof: finding a maximum matching now amounts to finding repeatedly a directed path. The proof yields a polynomial algorithm.

In the sequel we shall meet several other min-max relations which generalize König's matching theorem, like Menger's theorem on disjoint paths in directed graphs, the Tutte-Berge formula for matchings in arbitrary graphs, the perfect graph theorem, the Lucchesi-Younger theorem on directed cuts, the matroid intersection theorem.

By Gallai's theorem, König's matching theorem is equivalent to another result of König [1932].

Corollary 2a (König's covering theorem). The minimum number of edges covering al vertices of a bipartite graph is equal to the maximum size of a coclique, i.e., $\rho(G)=\alpha(G)$ for bipartite graphs $G$ (without isolated vertices).

Proof. Immediately by combining Theorems 1 and 2 .

It is characteristic for min-max relations that they produce, and often are equivalent to, a characterization of "necessary and sufficient" type. Thus, König's matching theorem yields that a bipartite graph $G=(V, E)$, with colour classes $V_{1}$ and $V_{2}$, has a matching covering $V_{1}$, if and only if $\left|\Delta\left(V^{\prime}\right)\right| \geqslant\left|V^{\prime}\right|$ for 
each subset $V^{\prime}$ of $V_{1}$. Here $\Delta\left(V^{\prime}\right)$ denotes the set of vertices adjacent to at least one vertex in $V^{\prime}$.

This corollary was found by Hall [1935] and is called Hall's Marriage theorem. In fact, it is not difficult to derive in turn König's theorem from Hall's. Hall's theorem is often formulated in terms of "transversals", "systems of distinct representatives", or "assignments" - see Mirsky [1971] for a comprehensive survey.

The theorems of König can be shown also with polyhedral methods. Let $A$ be the incidence matrix of the bipartite graph $G=(V, E)$. That is, the rows and columns of $A$ are indexed by $V$ and $E$, respectively, where $A_{v, e}=1$ or 0 according to whether or not $v$ belongs to $e$.

Then Theorem 2 and Corollary 2 a are equivalent to the LP-optima

$$
\begin{aligned}
& \max \{w x \mid x \geqslant 0, A x \leqslant b\}=\min \{y b \mid y \geqslant 0, y A \geqslant w\} \\
& \min \{w x \mid x \geqslant 0, A x \geqslant b\}=\max \{y b \mid y \geqslant 0, y A \leqslant w\}
\end{aligned}
$$

having integer optimum solutions, if $w$ and $b$ are all-one vectors.

Now it is not difficult to prove that $A$ is totally unimodular, and hence König's theorems follow also from Hoffman and Kruskal's result (cf. Section 3).

Of course, much more follows: for all integer vectors $w$ and $b$, the optima in (5) and (6) have integer optimum solutions. This gives more general min-max relations. E.g., it yields that, given a bipartite graph $G=(V, E)$ and a weight function $w: E \rightarrow \mathbb{Z}$,

(7) the maximum weight of a matching is equal to the minimum of $\sum_{v \in V} y(v)$ where $y: V \rightarrow \mathbb{Z}_{+}$such that $y(u)+y(v) \geqslant w(e)$ for all $e=\{u, v\}$ in $E$;

(8) the minimum weight of an edge-cover is equal to the maximum of $\sum_{v \in V} y(v)$ where $y: V \rightarrow \mathbb{Z}_{+}$such that $y(u)+y(v) \leqslant w(e)$ for all $e=\{u, v\}$ in $E$.

These results, due to Egerváry [1931], can also be shown algorithmically by extending the proof method of Theorem 2 above. This amounts to Kuhn's Hungarian method $[1955,1956]$ for the optimal assignment problem: given a matrix $\left(w_{i j}\right)_{i, j=1}^{n}$, find a permutation $\pi$ of $\{1, \ldots, n\}$ such that $\sum_{i=1}^{n} w_{i \pi(i)}$ is as large (or small) as possible.

Similarly, this gives an algorithm and a min-max relation for the (HitchcockKoopmans) transportation problem: given a cost matrix $\left(c_{i j}\right)_{i=1,}^{m}, i=1$, a "supply" vector $b=\left(b_{1}, \ldots, b_{m}\right)$ and a "demand" vector $d=\left(d_{1}, \ldots, d_{n}\right)$, find nonnegative integers $x_{i j}(i=1, \ldots, m ; j=1, \ldots, n)$ such that $\sum_{j=1}^{n} x_{i j} \leqslant b_{i}(i=1, \ldots, m)$ and $\sum_{i=1}^{m} x_{i j} \geqslant d_{j}(j=1, \ldots, n)$, and such that $\sum_{i, j} c_{i j} x_{i j}$ is as small as possible.

In fact, one of the most general min-max relations in this direction follows from the fact that, for a bipartite graph $G=(V, E)$, with incidence matrix $A$, and functions $b_{1}, b_{2}: V \rightarrow \mathbb{Z}, c_{1}, c_{2}, w: E \rightarrow \mathbb{Z}$, the optima 
(9) $\max \left\{w x \mid b_{1} \leqslant x \leqslant b_{2}, c_{1} \leqslant A x \leqslant c_{2}\right\}=$

$$
\min \left\{y_{2} c_{2}-y_{1} c_{1}+z_{2} b_{2}-z_{1} b_{1} \mid y_{1}, y_{2}, z_{1}, z_{2} \geqslant 0,\left(y_{1}-y_{2}\right) A+z_{2}-z_{1}=w\right\}
$$

have integer optimum solutions, again by the total unimodularity of $A$.

These integrality results can be formulated equivalently in terms of polyhedra. E.g., if $A$ again is the incidence matrix of the bipartite graph $G=(V, E)$, the fact that $\max \{w x \mid x \geqslant 0, A x \leqslant 1\}$ has an integer optimum solution for every $w$, is equivalent to the fact that the matching polytope of $G$ (which is the convex hull of the matchings in $G$ ) is determined by the constraints

$$
\begin{array}{ll}
x(e) \geqslant 0 & (e \in E), \\
x(\delta(v)) \leqslant 1 & (v \in V) .
\end{array}
$$

Similarly, the perfect matching polytope, being the convex hull of the perfect matchings, is determined by (10) after replacing $\leqslant$ in the second line by $=$. This is equivalent to a theorem of Birkhoff [1946] and Von Neumann [1953]: each doubly stochastic matrix is a convex combination of permutation matrices.

Edge-colourings. Moreover min-max relations have been found for edge-colourings in bipartite graphs, which seem not to follow from total unimodularity.

For any undirected graph $G=(V, E)$, let

(11) $\Delta(G)=$ the maximum degree of $G$,

$\chi(G)=$ the edge-colouring number of $G=$ the minimum number of colours needed to colour the edges of $G$ such that no two intersecting edges have the same colour.

Equivalently, $\chi(G)$ is the minimum number of matchings needed to cover the edges of $G$.

Clearly always $\Delta(G) \leqslant \chi(G)$, and again the triangle $K_{3}$ shows that strict inequality can occur. It is a famous theorem of Vizing [1964] that $\chi(G) \leqslant \Delta(G)+1$ if $G$ has no parallel edges. König [1916] showed that bipartite graphs again have $\Delta(G)=\chi(G)$.

Theorem 3 (König's edge-colouring theorem). The edge-colouring number of a bipartite graph $G$ is equal to its maximum degree, i.e., $\chi(G)=\Delta(G)$.

Proof. First notice that the theorem is easy if $\Delta(G) \leqslant 2$. In this case the graph consists of a number of vertex-disjoint paths and even circuits.

If $\Delta(G) \geqslant 3$, colour as many edges of $G$ as possible with $\Delta(G)$ colours, without giving the same colour to two intersecting edges. Suppose edge $e=\{u, v\}$ is not coloured. At least one colour, say red, does not occur among the colours given to the edges containing $v$. Similarly, there is a colour, say blue, not occurring at $w$. Clearly, red $\neq$ blue, since otherwise we could colour the edge $e$ red. Let $G^{\prime}$ be the subgraph of $G$ consisting of the red and blue edges, together with the edge $e$. Now $\Delta\left(G^{\prime}\right) \leqslant 2$, and hence $\chi\left(G^{\prime}\right) \leqslant 2$. So the edges occurring in $G^{\prime}$ 
may be recoloured red and blue, and in this way we have coloured more edges than before.

A related theorem was proved by Gupta $[1967,1978]$ and by D. König (unpublished).

Corollary 3a (Gupta's theorem). Let $G=(V, E)$ be a bipartite graph. Then the maximum number of pairwise disjoint edge-sets, each covering $V$, is equal to the minimum degree $\delta(G)$ of $G$.

Proof. One may derive from $G$ a bipartite graph $H$, with vertex-degrees $\delta(G)$ or 1, by repeated application of the following splitting procedure: choose a vertex $v$ of degree more than $\delta(G)$, add a new vertex $v^{\prime}$, and replace one edge $\{v, w\}$ by the new edge $\left\{v^{\prime}, w\right\}$ (the other edges containing $v$ remain unchanged). So the edges of the final graph $H$ are in one-to-one correspondence with the edges of G.

Since $H$ has maximum degree $\delta(G)$, by Theorem 3 the edges of $H$ can be coloured with $\delta(G)$ colours such that no two edges of the same colour intersect. It follows that at any vertex of $H$ of degree $\delta(G)$ all colours occur. In the original graph $G$ this yields $\delta(G)$ pairwise disjoint edge-covers as required.

If we understand the vertices of the two colour classes of a bipartite graph as teachers and as classes, respectively, and the edges as lessons to be given, we may interprete an edge-colouring as a time-table - each colour represents a period. For algorithms finding explicitly solutions, see De Werra [1970, 1972], Dempster [1971], McDiarmid [1972], Bondy and Murty [1976] pp. 96-100.

\section{Network Flows}

A second fundamental field of combinatorial min-max relations is that of flows in networks (directed graphs), with the max-flow min-cut theorem as landmark. Min-max relations for network flows are closely connected to those for bipartite graphs given in Section 4, and below we shall see that to a large extent they can be derived from each other by some simple constructions. For a survey on network flows, see Ford and Fulkerson [1962]. [1927].

The classical min-max equality for directed graphs is Menger's theorem

Theorem 4 (Menger's theorem). Let $D=(V, A)$ be a directed graph, and let $r$, $s \in V$. Then the maximum number of pairwise arc-disjoint $r$-s-paths is equal to the minimum size of an $r$-s-cut.

[An $r$-s-path is a directed path from $r$ to $s$. An $r$-s-cut is a set $\delta^{-}\left(V^{\prime}\right)$ with $r \notin V^{\prime}$, $\left.s \in V^{\prime}.\right]$

Proof. It is easy to see that the maximum is not more than the minimum. Like in the proof of König's matching theorem (Theorem 2), to prove the reverse 
inequality we describe a procedure which, given $k$ pairwise arc-disjoint $r$-spaths, either finds $k+1$ pairwise arc-disjoint $r$-s-paths, or finds an $r$-s-cut of size $k$.

Therefore, let $A_{1}, \ldots, A_{k}$ be the arc sets of the given $k$ pairwise arc-disjoint $r$-s-paths. Reverse the orientation of the arcs occurring in $A_{1} \cup \ldots \cup A_{k}$, thus making the directed graph $D^{\prime}$. Now there are two possibilities.

(i) There is an $r$-s-path in $D^{\prime}$. Let $A_{0}$ be the set of arcs of $D$ occurring in this path (reversed or not). Then it is easy to see that the symmetric difference $\left(A_{1} \cup \ldots \cup A_{k}\right) \Delta A_{0}$ is a disjoint union of $k+1$ arc-disjoint $r$-s-paths.

(ii) There is no $r$-s-path in $D^{\prime}$. Let $V^{\prime}$ be the set of points $v$ for which there exists a $v$-s-path in $D^{\prime}$. So $r \notin V^{\prime}, s \in V^{\prime}$. Moreover, $\delta_{\bar{A}}^{-}\left(V^{\prime}\right)$ contains not more than $k$ arcs. Indeed, no arc of $D^{\prime}$ enters $V^{\prime}$, so no $\operatorname{arc}$ in $A_{1} \cup \ldots \cup A_{k}$ leaves $V^{\prime}$, and no arc in $A \backslash\left(A_{1} \cup \ldots \cup A_{k}\right)$ enters $V^{\prime}$. Hence exactly one arc in each of the $A_{i}$ enters $V^{\prime}$, and no other arc of $D$ enters $V^{\prime}$. So $\left|\delta_{A}^{-}\left(V^{\prime}\right)\right|=k$.

This is again an algorithmic proof, based on the famous augmenting path method of Ford and Fulkerson [1956], and yielding a polynomial algorithm. The proof extends that of König's matching theorem - see also Remark 1 below.

The following corollaries can be seen to be equivalent to Menger's theorem.

Corollary 4 a (Menger's theorem - vertex form). Let $D=(V, A)$ be a directed graph, and let $r, s \in V$. Then the maximum number of pairwise internally vertexdisjoint $r$-s-paths is equal to the minimum number of vertices in $V \backslash\{r, s\}$ intersecting all r-s-paths.

Proof. By an easy construction from Theorem 4.

The second corollary is due to Dantzig [1951] (the existence of integer optimum flows), Ford and Fulkerson [1956] and Elias, Feinstein and Shannon [1956].

Corollary $4 \mathbf{b}$ (Max-flow min-cut theorem). Let $D=(V, A)$ be a directed graph, let $r, s \in V$ and let $c: A \rightarrow \mathbb{R}_{+}$be a capacity function. Then the maximum value of an $r$-s-flow subject to the capacity $c$ is equal to the minimum capacity of an r-s-cut. If all capacities are integer, there exists an integer optimum flow.

[Here an $r$-s-flow is a vector $x: A \rightarrow \mathbb{R}$ such that

$$
\begin{array}{ll}
\text { (i) } x(a) \geqslant 0 & (a \in A), \\
\text { (ii) } x\left(\delta^{-}(v)\right)=x\left(\delta^{+}(v)\right) & (v \in V, v \neq r, s) .
\end{array}
$$

The value of the flow is the net amount of flow leaving $r$, i.e., is

$$
x\left(\delta^{+}(r)\right)-x\left(\delta^{-}(r)\right)
$$

(which is equal to the net amount of flow entering $s$ ). The flow $x$ is subject to $c$ if $x(a) \leqslant c(a)$ for all $a$ in $A$.]

Proof. If the capacities are integer, the corollary follows from Menger's theorem by splitting each arc a into $c(a)$ parallel arcs, and by observing that $k$ 
pairwise arc-disjoint $r$-s-paths in the new graph yield an integer $r$-s-flow of value $k$ in the original graph, subject to $c$.

If $c$ is rational, there is a natural number $M$ such that $M c$ is integer. Since by multiplying $c$ by $M$, also the maximum flow value and the minimum cut capacity are multiplied by $M$, the case of $c$ rational follows from the case of $c$ integer.

If $c$ is real-valued, the corollary follows from the rational case by simple continuity and compactness arguments.

Note that this proof of splitting arcs is non-polynomial: if $c$ is integer, we obtain a graph of size proportional to $\sum_{a \in A} c(a)$, while the size of the original problem is proportional to $\sum_{a \in A} \log c(a)$. So the algorithm for Menger's theorem does not pass over in this way to a polynomial algorithm for max-flow min-cut. However, Ford and Fulkerson [1956] gave a direct maximum-flow algorithm, and Edmonds and Karp [1972] showed that this algorithm, with some modifications, is polynomial.

Above we saw that finding maximum packings of $r$-s-paths and finding $r$-sflows of maximum value, essentially are two sides of the same problem. In the following remark we shall see that bipartite matching is a third way of looking at this same problem.

Remark 1. König's matching theorem, and its algorithmic proof, described in Section 4, are special cases of Menger's theorem and its algorithmic proof given above. Indeed, if $G=(V, E)$ is a bipartite graph, with colour classes $V_{1}$ and $V_{2}$, orient the edges from $V_{1}$ to $V_{2}$, and add new vertices $r$ and $s$, and arcs $(r, v)$ for $v$ in $V_{1}$ and $(v, s)$ for $v$ in $V_{2}$. This makes the directed graph $D$. Then Menger's theorem for $D, r, s$ is equivalent to König's theorem for $G$.

In fact, also the converse implication holds by a direct construction, given by Orden [1956] (where the "transshipment problem" is reduced to the "transportation problem") and Hoffman [1960] (cf. Ford and Fulkerson [1958] and Hoffman and Markovitz [1963]). We here describe the idea behind this construction.

We show how to derive from König's matching theorem the following equivalent form of Menger's theorem: if $D=(V, A)$ is a directed graph, and $R$ and $S$ are disjoint subsets of $V$, then the maximum number of pairwise vertex-disjoint directed $R$-S-paths (i. e., paths starting in $R$ and ending in $S$ ), is equal to the minimum number of vertices intersecting all $R$-S-paths.

To this end, split each vertex $v$ of $D$ into two new vertices $v^{\prime}$ and $v^{\prime \prime}$, replace any $\operatorname{arc}(u, v)$ of $D$ by the $\operatorname{arc}\left(u^{\prime \prime}, v^{\prime}\right)$, and add $\operatorname{arcs}\left(v^{\prime \prime}, v^{\prime}\right)$ for $v$ in $V$. Denote for any subset $W$ of $V, W^{\prime}:=\left\{v^{\prime} \mid v \in W\right\}$ and $W^{\prime \prime}:=\left\{v^{\prime \prime} \mid v \in W\right\}$. Remove the vertices in $R^{\prime}$ and $S^{\prime \prime}$. This makes a (directed) bipartite graph $G$, with colour classes $V^{\prime \prime} \backslash S^{\prime \prime}$ and $V^{\prime} \backslash R^{\prime}$.

We show that if $k$ is the maximum number of pairwise vertex-disjoint $R-S$ paths in $D$, the matching number $v(G)$ of $G$ is equal to $|V|-|R|-|S|+k$, and that maximum packings of $R$-S-paths in $D$ and maximum matchings in $G$ can be transformed easily to each other. 
Let $A_{1}, \ldots, A_{k}$ be the arc sets of pairwise vertex-disjoint $R$-S-paths in $D$. Then the collection

$$
\begin{aligned}
& \left\{\left(u^{\prime \prime}, v^{\prime}\right) \mid(u, v) \in A_{1} \cup \ldots \cup A_{k}\right\} \cup\left\{\left(v^{\prime \prime}, v^{\prime}\right) \mid v \notin R \cup S,\right. \\
& \left.v \text { is not covered by } A_{1} \cup \ldots \cup A_{k}\right\}
\end{aligned}
$$

is a matching in $G$, of size $|V|-|R|-|S|+k$, and hence $v(G) \geqslant|V|-|R|-|S|+k$.

Conversely, let $M$ be a matching in $G$ of size $v(G)$. Consider the set $A^{\prime}$ of arcs $(u, v)$ of $D$ for which $\left(u^{\prime \prime}, v^{\prime}\right)$ belongs to $M$, and the set $V^{\prime}$ of vertices of $D$ for which $\left(v^{\prime \prime}, v^{\prime}\right)$ belongs to $M$. Then $A^{\prime}$ forms a collection of pairwise vertexdisjoint directed paths and cycles, not intersecting $V^{\prime}$. It is not difficult to see that at least $v(G)-|V|+|R|+|S|$ of these paths connect $R$ and $S$, and hence $v(G) \leqslant|V|-|R|-|S|+k$.

So finding a maximum packing of $R-S$-paths is equivalent to finding a maximum matching in $G$, and it is not difficult to check that König's theorem yields the form of Menger's theorem given above.

This may illustrate the third angle of vision: packings of paths can be considered alternatively not only as flows but also as matchings in an appropriate bipartite graph. Keeping this in mind is often helpful in understanding network flow problems.

Remark 2. As is well-known, the incidence matrix of a directed graph is totally unimodular, which also implies the max-flow min-cut theorem. Here the incidence matrix of the directed graph $D=(V, A)$ is the matrix $H$ with rows and columns indexed by $V$ and $A$, respectively, and with

$$
\begin{aligned}
H_{v, a}=+1 & \text { if } a=(v, u) \text { for some } u, \\
-1 & \text { if } a=(u, v) \text { for some } u, \\
0 & \text { otherwise, }
\end{aligned}
$$

for $v \in V, a \in A$

Indeed, if $r, s \in V$, let $D_{0}=\left(V, A_{0}\right)$ arise from $D$ by adding the arc $(s, r)$. Let $H_{0}$ be the incidence matrix of $D_{0}$. Then $H_{0}$ is totally unimodular, and hence for all $c, w: A_{0} \rightarrow \mathbb{Z}_{+}$, both sides of the LP-duality equation

$$
\max \left\{w x \mid 0 \leqslant x \leqslant c, H_{0} x=0\right\}=\min \left\{y c \mid y \geqslant 0, y+z H_{0}=w \text { for some } z \in \mathbb{R}^{V}\right\}
$$

have integer optimum solutions. Taking $w(s, r)=1$, and $w(a)=0$ for $a$ in $A$, and $c(s, r)=\infty$ (or very large), gives the max-flow min-cut theorem. Below we shall see more applications.

There is an alternative way of viewing max-flow min-cut in terms of linear programming. Let $D=(V, A)$ be a directed graph, and let $r, s \in V$. Let $K$ be the matrix with columns indexed by $A$, and with rows all incidence vectors of $r$-spaths (considered as arc sets). Then the max-flow min-cut theorem says that for $c: A \rightarrow \mathbb{R}_{+}$, the minimum in

$$
\min \{c x \mid x \geqslant 0, K x \geqslant 1\}=\max \{|y| \mid y \geqslant 0, y K \leqslant c\}
$$


has an integer optimum solution. If moreover $c$ is integer, also the maximum has an integer optimum solution.

Integer optimum solutions for the minimum in (6) are $\{0,1\}$-vectors, which are exactly incidence vectors of $r$-s-cuts. Solutions for the maximum in (6) correspond to $r$-s-flows subject to $c$.

Here we could apply the ellipsoid method: the minimum in (6) can be found in polynomial time, as the inequality system $x \geqslant 0, K x \geqslant 1$ can be checked in polynomial time. Indeed, checking $x \geqslant 0$ is easy. To check $K x \geqslant 1$ we cannot enumerate all inequalities as there may exist exponentially many. However, for given $x \geqslant 0$ the system $K x \geqslant 1$ can be checked as follows. Consider $x$ as a length function, and find an $r$-s-path of minimum length (e.g., by Dijkstra's method). If this length is less than 1 this path gives a violated inequality in $K x \geqslant 1$. Otherwise we conclude that $K x \geqslant 1$ holds.

This application of the ellipsoid method is maybe not very convincing: the formulation (5) allows a direct application of Khachiyan's method to a concrete (polynomially bounded) LP-problem. In the next sections we shall see more satisfactory applications of the ellipsoid method, which extend the idea of (6).

There is an easier theorem, which is in a sense "polar" to the results above. Again this result can be formulated equivalently in combinatorial terms and in terms of "currents". The latter one we give first.

Theorem 5 (Min-potential max-work theorem). Let $D=(V, A)$ be a directed graph, let $r, s \in V$, and let $l: A \rightarrow \mathbb{R}_{+}$be a length function. Then the maximum potential difference of $r$ and $s$ is equal to the minimum length of an $r$-s-path. Moreover, if all length are integer, there is an integer optimum potential.

Here a potential is a function $\phi: V \rightarrow \mathbb{R}$ such that $\phi(v)-\phi(u) \leqslant l(a)$ for $a=(u, v)$ in $A$. The potential difference of $r$ and $s$ is $\phi(s)-\phi(r)$.

Proof. The inequality $\max \leqslant \min$ is trivial. Defining $\phi(v):=$ the minimum length of an $r$-v-path gives a potential for which $\phi(s)-\phi(r)$ equals the minimum length of an $r$-s-path.

E.g. with Dijkstra's method one easily handles the optima of Theorem 5 algorithmically.

The equivalent combinatorial version is as follows.

Corollary 5 a. Let $D=(V, A)$ be a directed graph, and let $r, s \in V$. Then the minimum number of arcs in an r-s-path is equal to the maximum number of pairwise disjoint r-s-cuts.

Proof. Apply Theorem 5 with $l=1$. Let $\phi$ be a potential with $\phi(s)-\phi(r)$ equal to the minimum number of arcs in an $r$-s-path. If $k$ is this minimum number, let $V_{i}:=\{v \in V \mid \phi(v)>\phi(s)-i\}$ for $i=1, \ldots, k$. Then $\delta^{-}\left(V_{1}\right), \ldots, \delta^{-}\left(V_{k}\right)$ are pairwise disjoint $r$-s-cuts.

This theorem, observed by Fulkerson [1968], is "polar" to Menger's theorem: interchanging " $r$-s-paths" and " $r$-s-cuts" carries one to the other. Similarly as in Remark 2 above, the max-potential min-work theorem can be formu- 
lated in terms of linear programming in two ways, which is left to the reader. It will follow that, by the theory of blocking polyhedra, the max-flow min-cut theorem and the max-potential min-work theorem are equivalent, if we forget the integrality of optimum flows and potentials.

Remark 3. By the Bellman-Ford method one can solve also the shortest path problem if lengths are allowed to be negative, provided that all directed cycles have nonnegative length. A corresponding min-max relation and good characterization can be derived from Theorem 5 as follows.

Let $D=(V, A)$ be a directed graph, and let $l: A \rightarrow \mathbb{R}$ be a length function such that each directed cycle has a nonnegative length. Define, for each vertex $v$ of $D, \psi(v)$ to be the shortest length of any directed path ending in $v$ (starting whereever). Then for each $\operatorname{arc}(u, v)$ of $D, l(u, v) \geqslant \psi(v)-\psi(u)$, and hence $\tilde{l}(u, v):=l(u, v)+\psi(u)-\psi(v)$ is nonnegative. However, the shortest path problem for $l$ is equivalent to that for $\tilde{l}$.

Note that the shortest path problem for undirected graphs is easily reduced to the directed case if all lenghts are nonnegative: replace each edge $\{u, v\}$ by two arcs $(u, v)$ and $(v, u)$ of the same length as $\{u, v\}$. However, if edges have a negative length, we would create a negative directed cycle in this way. Yet there is a min-max relation and a polynomial algorithm for the shortest path problem in undirected graphs, also if negative lengths occur, provided that there are no circuits of negative length - see the subsection on $T$-joins in Section 6.

Actually, the max-flow min-cut and max-potential min-work theorems can be combined to one min-max relation for minimum-cost flows. A combinatorial version of this theorem is as follows.

Theorem 6 (Min-cost flow theorem - combinatorial version). Let $D=(V, A)$ be a directed graph, let $r, s \in V$, and let $k \in \mathbb{Z}_{+}$. Then the minimum number of arcs in $k$ pairwise arc-disjoint $r$-s-paths is equal to

$$
\max \left(k t+\left|A_{1} \cup \ldots \cup A_{t}\right|-\sum_{i=1}^{t}\left|A_{i}\right|\right),
$$

where the maximum ranges over all $t \geqslant 0$ and collections of $r$-s-cuts $A_{1}, \ldots, A_{t}$.

This theorem contains Corollary $5 \mathrm{a}$ on shortest paths by taking $k=1$. It also contains Menger's theorem: the largest $k$ for which there exist $k$ pairwise arc-disjoint $r$-s-paths is equal to the largest $k$ for which the maximum (7) is bounded, which can be seen to be the minimum size of an $r$-s-cut.

Theorem 6 in its flow-formulation follows from the total unimodularity of the incidence matrix $H$ of $D$. This gives that both optima in:

$$
\min \left\{w x \mid c_{1} \leqslant x \leqslant c_{2}, H x=0\right\}=\max \left\{y_{1} c_{1}-y_{2} c_{2} \mid y_{1}, y_{2} \geqslant 0, y_{1}-y_{2}+z H=w\right\}
$$

have integer optimum solutions, for $w, c_{1}, c_{2}: A \rightarrow \mathbb{Z}$. By choosing $w, c_{1}, c_{2}$ appropriately, (8) gives a min-max relation for the minimum cost of an $r$-s-flow subject to a capacity constraint, and of a given value. It also follows that if the capacities are integer, there is an integer minimum-cost flow. This contains 
(and is actually equivalent to) Theorem 6 by taking all costs and capacities equal to one.

Fulkerson [1961] and Minty [1960] designed an algorithm for finding a minimum-cost flow. This out-of-kilter method also produces the min-max relation. Edmonds and Karp [1972] gave a polynomial-time version of this method.

Similarly the minimum-cost circulation problem can be treated, which consists of finding optimum solutions $x$ and $y$ for (8) (in its full generality). Here a circulation is a function $x: A \rightarrow \mathbb{R}$ satisfying

$$
x\left(\delta^{-}(v)\right)=x\left(\delta^{+}(v)\right)
$$

for all $v$ in $V$. (Often one adds the condition $x \geqslant 0$, so that a circulation can be considered as an " $r$ - $r$-flow", for arbitrary vertex $r$.)

The out-of-kilter method finds integer optimum circulations $x$ if the "need" function $c_{1}$ and the capacity function $c_{2}$ are integer, and it finds an integer optimum solution $y_{1}, y_{2}$ if the cost function $w$ is integer.

The min-max relation (8) for minimum-cost circulations also contains the following theorem of Hoffman [1960]: given a directed graph $D=(V, A)$ and a need function $c_{1}: A \rightarrow \mathbb{R}$ and a capacity function $c_{2}: A \rightarrow \mathbb{R}$ with $c_{1} \leqslant c_{2}$, there exists a circulation $x$ satisfying $c_{1} \leqslant x \leqslant c_{2}$ if and only if for each subset $V^{\prime}$ of $V$ the capacity of $\delta^{-}\left(V^{\prime}\right)$ is not less than the need of $\delta^{+}\left(V^{\prime}\right)$. [Note that this last condition is eqivalent to: if $y_{1}, y_{2}: A \rightarrow \mathbb{Z}_{+}$and $z: V \rightarrow \mathbb{Z}$ such that $y_{1}-y_{2}+z H=0$, then $y_{1} c_{1} \leqslant y_{2} c_{2}$. So Hoffman's theorem follows from the fact that the minimum in (8) is feasible (for $w=0$ ), if and only if the maximum in (8) is bounded (as it is obviously feasible).] If moreover $c_{1}$ and $c_{2}$ are integer, the polyhedron $\left\{x \mid c_{1} \leqslant x \leqslant c_{2}, H x=0\right\}$ has integer vertices, and hence the existence of a circulation between $c_{1}$ and $c_{2}$ implies the existence of an integer circulation between $c_{1}$ and $c_{2}$.

We finally give one of the most general min-max relations in this direction, which again follows from total unimodularity. If $H$ is the incidence matrix of the directed graph $D=(V, A)$, and if $b_{1}, b_{2}: V \rightarrow \mathbb{R}$ and $c_{1}, c_{2}, w: A \rightarrow \mathbb{R}$, then

$$
\begin{aligned}
& \min \left\{w x \mid c_{1} \leqslant x \leqslant c_{2}, b_{1} \leqslant H x \leqslant b_{2}\right\}= \\
& \max \left\{y_{1} c_{1}-y_{2} c_{2}+z_{1} b_{1}-z_{2} b_{2} \mid y_{1}, y_{2}, z_{1}, z_{2} \geqslant 0, y_{1}-y_{2}+\left(z_{1}-z_{2}\right) H=w\right\} .
\end{aligned}
$$

If $b_{1}, b_{2}, c_{1}, c_{2}$ are integer the minimum has an integer optimum solution. If $w$ is integer, the maximum has an integer optimum solution.

This min-max relation contains all min-max results described above. It may be derived in turn form the min-max relation (8) for minimum-cost circulations, by some direct constructions. These constructions also give that the optimum solutions in (10) can be found with Fulkerson and Minty's out-of-kilter method for the min-cost circulation problem:

A wide range of further min-max equalities are contained in (10) as special cases, e.g., for flows obeying lower bounds, and for maximum-"gain" flows. Also the following min-max relations for partially ordered sets can be derived from (10). 
Theorem 7 (Dilworth's theorem). The maximum size of an antichain in a partially ordered set $(P, \leqslant)$ is equal to the minimum number of chains needed to cover $P$.

[Dilworth [1950], cf. Fulkerson [1956]. An (anti)chain is a set of pairwise (in)comparable elements. In fact, Dilworth's theorem is equivalent to König's matching theorem by a similar construction as the one described in Remark 1.]

Theorem 8. The maximum size of a chain in a partially ordered set $(P, \leqslant)$ is equal to the minimum number of antichains needed to cover $P$.

[This is an easy theorem.]

Theorem 9 (Greene-Kleitman theorem). The maximum size of the union of $k$ antichains in a partially ordered set $(P, \leqslant)$ is equal to $\min _{P^{\prime} \subseteq P}\left|P \backslash P^{\prime}\right|+k \gamma\left(P^{\prime}\right)$, where $\gamma\left(P^{\prime}\right)$ denotes the minimum number of chains needed to cover $P^{\prime}$.

[Greene and Kleitman [1976], cf. Hoffman and Schwartz [1977], Frank [1980], Cameron [1982].]

Theorem 10 (Greene's theorem). The maximum size of the union of $k$ chains in a partially ordered set $(P, \leqslant)$ is equal to $\min _{P^{\prime} \subseteq P}\left|P \backslash P^{\prime}\right|+k \alpha\left(P^{\prime}\right)$, where $\alpha\left(P^{\prime}\right)$ denotes the minimum number of antichains needed to cover $P^{\prime}$.

[Greene [1976], cf. Hoffman and Schwartz [1977], Frank [1980], Cameron [1982]. This theorem is the poset analogue of Theorem 6 on unions of paths.]

By applying a projection technique, Cameron [1982] derived the following result from the total unimodularity of the incidence matrix of a directed graph. Let $D=(V, A)$ be a directed graph, and let $c_{1}, c_{2}, d: A \rightarrow \mathbb{Z}$. Then the following system is totally dual integral:

$$
\begin{array}{ll}
c_{1}(a) \leqslant x(a) \leqslant c_{2}(a) & (a \in A), \\
x(C) \leqslant d(C) & (C \subseteq A, C \text { directed cycle }) .
\end{array}
$$

From this result again Dilworth's theorem and the theorems of Greene and Kleitman follow. Also the perfectness of certain graphs is included - see Section 9.

\section{Matchings and Generalizations}

In Section 4 we discussed matching and covering problems for bipartite graphs. We now consider these problems for the more difficult general case of not-necessarily bipartite graphs.

The fundamental result on matchings is Tutte's perfect matching theorem [1947], characterizing undirected graphs containing a perfect matching. Tutte [1952], 1954] and Edmonds and Johnson [1973] showed that this theorem is of a self-refining nature: by a series of elementary constructions it can be generalized to results on objects like (capacitated) $b$-matchings, Chinese postman 
routes, and " $T$-joins". Below we emphasize describing these constructions, rather than deriving the min-max relations in detail.

Matchings and coverings. Berge [1958] showed that Tutte's perfect matching theorem is equivalent to the following min-max relation for the maximum matching size.

Theorem 11 (Tutte-Berge formula). Let $G=(V, E)$ be an undirected graph. Then the maximum size $v(G)$ of a matching in $G$ is equal to

$$
\min _{V^{\prime} \subseteq V} \frac{|V|+\left|V^{\prime}\right|-\odot\left(V \backslash V^{\prime}\right)}{2}
$$

where $\mathscr{O}\left(V \backslash V^{\prime}\right)$ denotes the number of components of odd size in the graph $\left\langle V \backslash V^{\prime}\right\rangle$ induced by $V \backslash V^{\prime}$.

Proof. The inequality $\max \leqslant \min$ is an easy exercise (observe that for $V^{\prime} \subseteq V$, any matching leaves at least $\mathscr{O}\left(\backslash V^{\prime}\right)-\left|V^{\prime}\right|$ vertices uncovered). Equality is proved by induction on $|V|$, where $V=\emptyset$ is trivial. Consider the following two cases.

(i) There is a vertex $v$ covered by every matching of size $v(G)$. Let $G^{\prime}$ arise from $G$ by deleting $v$ and the edges containing $v$. Then $v\left(G^{\prime}\right)=v(G)-1$, and moreover, by induction, $\nu\left(G^{\prime}\right)=\frac{1}{2}\left(|V \backslash v|+\left|V^{\prime \prime}\right|-\odot\left((V \backslash v) \backslash V^{\prime \prime}\right)\right)$ for some $V^{\prime \prime} \subseteq V \backslash v$. Taking $V^{\prime}:=V^{\prime \prime} \cup v$ gives $v(G)=v\left(G^{\prime}\right)+1=\frac{1}{2}\left(|V|+\left|V^{\prime}\right|-\mathscr{O}\left(V \backslash V^{\prime}\right)\right)$, and we have the required equality.

(ii) No vertex is covered by every matching of size $v(G)$. We show that each matching of size $v(G)$ leaves at most one vertex in each of the components of $G$ uncovered. This will prove equality in (1), since then $2 v(G)=|V|-\mathscr{O}(V)$, and we can take $V^{\prime}=\emptyset$ in (1).

Suppose to the contrary there are two vertices $u$ and $v$ in one component of $G$ not covered by the maximum matching $M$, and assume furthermore that we have chosen $M$ and $u$ and $v$ such that $u$ and $v$ have distance $d(u, v)$ as small as possible.

Obviously, $d(u, v)>1$, since otherwise we could augment $M$ by the edge $\{u, v\}$. Let $w \neq u, v$ be a vertex on the shortest $u-v$-path, and let $M^{\prime}$ be a maximum matching not covering $w$. Then $M \cup M^{\prime}$ forms a vertex-disjoint union of paths and circuits. Let $P$ be the set of edges in the component of $M \cup M^{\prime}$ containing $w$. Then $P$ forms a path, starting in $w$, and not containing both $u$ and $v$ (as each of $u, v$ and $w$ is covered by at most one edge in $M \cup M^{\prime}$ ). Say $P$ does not cover $u$. Then the symmetric difference $M \Delta P$ again is a maximum matching (as $|M \Delta P|-|M|=\left|M^{\prime}\right|-\left|M^{\prime} \Delta P\right| \geqslant 0$ ), not covering $u$ and $w$. However, $d(u, w)<d(u, v)$, contradicting our choice of $M, u, v$.

It is not difficult to see that the Tutte-Berge formula generalizes König's matching theorem.

Combining the Tutte-Berge formula with Gallai's theorem (Theorem 1) directly gives the following. 
Corollary 11 a. Let $G=(V, E)$ be an undirected graph without isolated vertices. Then the minimum number $\rho(G)$ of edges covering $V$ is equal to

$$
\max _{V^{\prime} \subseteq V} \frac{\left|V^{\prime}\right|+\mathscr{O}\left(V^{\prime}\right)}{2} \text {. }
$$

Proof. Directly from Theorems 1 and 11.

Another consequence is the original perfect matching theorem of Tutte [1947]: an undirected graph $G=(V, E)$ has a perfect matching, if and only if for each subset $V^{\prime}$ of $V$, the size of $V^{\prime}$ is not less than the number of odd-sized components in the subgraph induced by $V \backslash V^{\prime}$.

The proof of the Tutte-Berge theorem given above, containing ideas described in Lovász [1979a], is an example of a strictly combinatorial proof, seemingly not implying directly a polynomial algorithm for finding a maximum matching. Edmonds [1965c] designed a polynomial algorithm finding a maximum matching, which yields as a by-product the Tutte-Berge theorem.

More generally, Edmonds [1965d] gave a polynomial algorithm finding a maximum weighted matching. This algorithm gives as a by-product Edmonds' matching polytope theorem: the matching polytope of $G=(V, E)$ (being the convex hull of the matchings in $G$ ) is determined by the inequalities

$$
\begin{array}{ll}
\text { (i) } x(e) \geqslant 0 & (e \in E), \\
\text { (ii) } x(\delta(v)) \leqslant 1 & (v \in V), \\
\text { (iii) } x\left(\left\langle V^{\prime}\right\rangle\right) \leqslant\left\lfloor\frac{1}{2}\left|V^{\prime}\right|\right\rfloor & \left(V^{\prime} \subseteq V,\left|V^{\prime}\right| \text { odd }\right) .
\end{array}
$$

Here $\left\langle V^{\prime}\right\rangle$ is the set of edges contained in $V^{\prime}$, and $\left.L\right\lrcorner$ denotes lower integer part.

The incidence vector of any matching clearly satisfies (3), and hence the matching polytope is contained in (3) - the content of Edmonds' theorem is the converse inclusion.

The matching polytope theorem is equivalent to the following. Denote the system (3) (ii) by $A x \leqslant 1$, and the system (3) (iii) by $B x \leqslant f$. So the columns of $A$ and $B$ are indexed by $E$, and their rows by $V$ and by the odd subsets of $V$, respectively. Then the maximum in the LP-duality equation

(4) $\max \{w x \mid x \geqslant 0, A x \leqslant 1, B x \leqslant f\}=\min \{|y|+z f \mid y \geqslant 0, z \geqslant 0, y A+z B \geqslant w\}$

has an integer optimum solution (being a matching). The inequalities (3) (iii) can be considered as the Gomory cuts, the addition of which to (3) (i) (ii) transforms the integer linear program to a linear program.

So Edmonds' theorem is equivalent to a min-max relation for the maximum weight of a matching. Cunningham and Marsh [1979] showed that for integer $w$ also the minimum in (4) has an integer optimum solution, i. e., that the system (3) is totally dual integral. This extends the Tutte-Berge theorem, which is the case $w=1$.

The proof by Cunningham and Marsh is algorithmical. Schrijver and Seymour [1977] (cf. Schrijver [1981]) gave a polyhedral proof. We here give a combinatorial proof which extends the above proof of the Tutte-Berge theorem. 
Theorem 12. For integer $w$, both optima in (4) have integer optimum solutions.

Proof. For $G=(V, E)$ and $w \in \mathbb{Z}_{+}^{E}$, let $v_{w}$ denote the maximum weight of a matching. We have to show that there are integer vectors $y \geqslant 0$ and $z \geqslant 0$ such that $y A+z B \geqslant w$ and $v_{w} \geqslant|y|+z f$.

Suppose $G$ and $w$ contradict this, with $|V|+|E|+w(E)$ as small as possible. Then $G$ is connected (otherwise one of the components of $G$ will form a smaller counterexample), and $w(e) \geqslant 1$ for each edge $e$ (otherwise we could delete $e$ ).

Now there are two cases.

(i) There is a vertex $v$ covered by every maximum-weighted matching. In this case, let $w^{\prime} \in \mathbb{Z}_{+}^{E}$ arise from $w$ by decreasing the weights of the edges containing $v$ by one. Then $v_{w^{\prime}}=v_{w}-1$. Since $w^{\prime}(E)<w(E)$, there are integer vectors $y^{\prime} \geqslant 0, z \geqslant 0$ such that $y^{\prime} A+z B \geqslant w^{\prime}$ and $\left|y^{\prime}\right|+z f \leqslant v_{w^{\prime}}$. Increasing $y_{v}^{\prime}$ by one gives $y \geqslant 0, z \geqslant 0$ such that $y A+z B \geqslant w$ and $|y|+z f \leqslant v_{w}$.

(ii) No vertex is covered by every maximum-weighted matching. Now let $w^{\prime}$ arise from $w$ by decreasing all weights by one. We show that $\nu_{w^{\prime}} \leqslant v_{w}-\left\lfloor\frac{1}{2}|V|\right\rfloor$. This will imply the theorem: since $w^{\prime}(E)<w(E)$ there are integer vectors $y \geqslant 0$, $z^{\prime} \geqslant 0$ such that $y A+z^{\prime} B \geqslant w^{\prime}$ and $|y|+z^{\prime} f \leqslant v_{w^{\prime}}$; increasing $z_{V}^{\prime}$ by one gives integer vectors $y \geqslant 0, z \geqslant 0$ such that $y A+z B \geqslant w$ and $|y|+z f \leqslant v_{w}$.

Assume $v_{w^{\prime}}<v_{w^{\prime}}+\left\lfloor\frac{1}{2}|V|\right\rfloor$, and let $M$ be a matching with $v_{w^{\prime}}=w^{\prime}(M)$, such that $w(M)$ is as large as possible. Then $M$ leaves at least two vertices in $V$ uncovered, since otherwise $v_{w} \geqslant w(M) \geqslant w^{\prime}(M)+\left\lfloor\frac{1}{2}|V|\right\rfloor=v_{w},+\left\lfloor\frac{1}{2}|V|\right\rfloor$.

Let $u$ and $v$ be not covered by $M$, and suppose we have chosen $M, u$ and $v$ such that the distance $d(u, v)$ is as small as possible. Then $d(u, v)>1$, since otherwise we could augment $M$ by $\{u, v\}$, thereby increasing $w(M)$. Let $t$ be an internal vertex of the shortest path between $u$ and $v$. Let $M^{\prime}$ be a matching with $w\left(M^{\prime}\right)=v_{w^{\prime}}$ not covering $t$.

Now $M \cup M^{\prime}$ is a disjoint union of paths and circuits. Let $P$ be the set of edges of the component of $M \cup M^{\prime}$ containing $t$. Then $P$ forms a path starting in $t$ and not covering both $u$ and $v$ (as $t, u$ and $v$ have degree at most one in $\left.M \cup M^{\prime}\right)$. Say $P$ does not cover $u$. Now the symmetric difference $M \Delta P$ is a matching with $|M \Delta P| \leqslant|M|$, and therefore:

$$
\begin{aligned}
w^{\prime}(M \Delta P)-w^{\prime}(M) & =w(M \Delta P)-|M \Delta P|-w(M)+|M| \\
& \geqslant w(M \Delta P)-w(M)=w\left(M^{\prime}\right)-w\left(M^{\prime} \Delta P\right) \geqslant 0 .
\end{aligned}
$$

Hence $v_{w^{\prime}}=w^{\prime}(M \Delta P)$ and $w(M \Delta P) \geqslant w(M)$. However, $M \Delta P$ does not cover $t$ and $u$, and $d(u, t)<d(u, v)$, contradicting our choice of $M, u, v$.

Edmonds' weighted matching algorithm can be easily modified to find minimum (or maximum) weighted perfect matchings in polynomial time. Moreover, the characterization (3) of the matching polytope directly gives that the perfect matching polytope (being the convex hull of the perfect matchings) is determined by:

$$
\begin{array}{ll}
\text { (i) } x(e) \geqslant 0 & (e \in E), \\
\text { (ii) } x(\delta(v))=1 & (v \in V), \\
\text { (iii) } x\left(\delta\left(V^{\prime}\right)\right) \geqslant 1 & \left(V^{\prime} \subseteq V,\left|V^{\prime}\right| \text { odd }\right)
\end{array}
$$


Indeed, adding $x(E)=\frac{1}{2}|V|$ to (3) is equivalent to (6).

With LP-duality this gives a min-max relation for the minimum and for the maximum weight of a perfect matching. It follows from Theorem 12 that the system (6) is totally dual half-integral. The graph $K_{4}$ shows that (6) generally is not totally dual integral. Multiplying (6) (iii) by $\frac{1}{2}$ would make the system totally dual integral.

The polynomial solvability of the weighted (perfect) matching problem also follows with the ellipsoid method: Padberg and Rao [1982] showed that the inequalities (6) can be checked in polynomial time (by reduction to a series of minimum-cut problems), and hence the ellipsoid method gives polynomial solvability.

Similar results hold for weighted edge-covers.

$\boldsymbol{b}$-Matchings. The results described above are of a certain self-refining nature. Given an undirected graph $G=(V, E)$ and a function $b: V \rightarrow \mathbb{Z}_{+}$, a $b$-matching is a vector $x: E \rightarrow \mathbb{Z}_{+}$such that $x(\delta(v)) \leqslant b(v)$ for all $v$ in $V$. If we have equality here for each $v$, the $b$-matching is called perfect. So if $b \equiv 1, b$-matchings reduce to ordinary matchings.

In case $b \equiv 2$, we obtain 2-matchings, which may be considered as a collection of vertex-disjoint paths and circuits (corresponding to edges $e$ with $x(e)=1$ ), and edges (corresponding to edges $e$ with $x(e)=2$ ). Define a 2-vertexcover as a function $y: V \rightarrow \mathbb{Z}_{+}$such that $y(u)+y(v) \geqslant 2$ for each edge $\{u, v\}$ of $G$, and define the size of a vector to be the sum of its components. Now Tutte [1952, 1953] showed:

(7) in any undirected graph, the maximum size of a 2-matching is equal to the minimum size of a 2-vertex-cover.

This can be derived from König's matching theorem for bipartite graphs (Theorem 2) by replacing each vertex $v$ of $G$ by two new vertices $v^{\prime}$ and $v^{\prime \prime}$, and each edge $\{u, v\}$ by two new edges $\left\{u^{\prime}, v^{\prime \prime}\right\}$ and $\left\{u^{\prime \prime}, v^{\prime}\right\}$, and by applying König's theorem to the bipartite graph thus obtained.

One may derive similarly a min-max relation for "2-edge-covers" and " 2 cocliques", and an associated Gallai-type theorem - see Lovász [1975].

The case of arbitrary $b$ can be reduced to the Tutte-Berge theorem, which gives that the maximum size of a $b$-matching is equal to

$$
\min _{V^{\prime} \subseteq V} \frac{b(V)+b\left(V^{\prime}\right)-\beta\left(V \backslash V^{\prime}\right)}{2}
$$

where $\beta\left(V \backslash V^{\prime}\right)$ denotes the following: let $I$ be the set of isolated vertices in the graph $\left\langle V \backslash V^{\prime}\right\rangle$ induced by $V \backslash V^{\prime}$, and let $t$ be the number of components $C$ of the graph $\left\langle V \backslash\left(V^{\prime} \cup I\right)\right\rangle$ with $b(C)$ odd; then $\beta\left(V \backslash V^{\prime}\right):=b(I)+t$ (cf. Tutte [1952]).

To derive this formula from Theorem 11 , split each vertex $v$ of $G$ into $b(v)$ new vertices, and replace any edge $\{u, v\}$ of $G$ by $b(u) b(v)$ new edges connecting the new vertices corresponding to $u$ and those corresponding to $v$. Next apply Theorem 11 to the new graph. This construction was given by Tutte [1954]. The min-max relation implies the original characterization of Tutte [1952] of graphs with a perfect $b$-matching. 
Edmonds and Pulleyblank (cf. Pulleyblank [1973]) showed that the $b$-matching polytope (being the convex hull of the $b$-matchings) is determined by the inequalities

$$
\begin{array}{rll}
\text { (i) } & x(e) \geqslant 0 & (e \in E), \\
\text { (ii) } & x(\delta(v)) \leqslant b(v) & (v \in V), \\
\text { (iii) } & x\left(\left\langle V^{\prime}\right\rangle\right) \leqslant\left\lfloor\frac{1}{2} b\left(V^{\prime}\right)\right\rfloor & \left(V^{\prime} \subseteq V\right) .
\end{array}
$$

This can be derived from characterization (3) of the matching polytope by the same method of splitting of vertices. Clearly, characterization (9) gives a minmax relation for the weighted $b$-matching problem. Pulleyblank [1980] strengthened this relation by showing that (9) is totally dual integral.

The method of splitting vertices described above does not carry over the polynomial solvability from the matching problem to the $b$-matching problem: the size of the $b$-matching problem is proportional to $\sum_{v \in V} \log b(v)$, while splittings create a graph of size proportional to $\sum_{v \in V} b(v)$. Such a splitting preserves polynomiality if $b(v)$ is bounded, e. g., in the case of 2-matchings.

Cunningham and Marsh (cf. Marsh [1979]) showed that also the general maximum weighted $b$-matching problem can be solved in polynomial time. Again, the polynomial solvability can be derived alternatively from the characterization (9) with the ellipsoid method - see Padberg and Rao [1982].

Perfect $b$-matchings can be treated similarly. It follows from (9) that the perfect b-matching polytope (being the convex hull of the perfect $b$-matchings) is determined by

$$
\begin{array}{ll}
\text { (i) } x(e) \geqslant 0 & (e \in E) \\
\text { (ii) } x(\delta(v))=b(v) & (v \in V), \\
\text { (iii) } x\left(\delta\left(V^{\prime}\right)\right) \geqslant 1 & \left(V^{\prime} \subseteq V, b\left(V^{\prime}\right) \text { odd }\right) .
\end{array}
$$

So this gives a min-max relation for the weighted perfect $b$-matching problem. Generally (10) is not totally dual integral. However, replacing (10) (iii) by (9) (iii) makes (10) totally dual integral.

In particular, the perfect 2-matching polytope is determined by:

$$
\begin{array}{ll}
\text { (i) } x(e) \geqslant 0 & (e \in E), \\
\text { (ii) } x(\delta(v))=2 & (v \in V),
\end{array}
$$

and this system is totally dual integral, after adding the constraints $x\left(\left\langle V^{\prime}\right\rangle\right) \leqslant\left|V^{\prime}\right|$ for $V^{\prime} \subseteq V$. A perfect 2-matching may be considered as a vertexdisjoint union of circuits and "double" edges, covering $V$.

The minimum weight perfect 2-matching problem is sometimes used as a relaxation for the traveling salesman problem (cf. Balas and Christofides [1981]). Here one would like to dispose of small circuits in the perfect 2-matching (in fact, of all circuits smaller than $|V|)$. While removing "double" edges gives more problems (see below), Cornuéjols and Pulleyblank [1980] showed that just adding to (11) the constraints

$$
x(\{u, v\})+x(\{v, w\})+x(\{u, w\}) \leqslant 2
$$


for all triples $u, v, w$ gives the convex hull of the perfect 2-matchings without triangles. Also total dual integrality is maintained if we add again $x\left(\left\langle V^{\prime}\right\rangle\right) \leqslant\left|V^{\prime}\right|\left(V^{\prime} \subseteq V\right)-$ cf. Cook and Pulleyblank [1982].

Capacitated b-matchings. Frequently when considering $b$-matching problems we wish that the $b$-matchings contain certain edges not too often, e.g. when using 2-matchings as relaxation for traveling salesman routes. It turns out that min-max results for such capacitated b-matchings can be derived from those for uncapacitated $b$-matchings given above, by an elementary construction given by Tutte [1954]. This shows again the self-refining nature of matching theory.

We first consider the 2-matching case. Let $G=(V, E)$ be an undirected graph. Call a 2-matching $x$ simple if $x \leqslant 1$, i. e., if $x$ is a $\{0,1\}$-vector with $x(\delta(v)) \leqslant 2$ for each vertex $v$. A simple 2 -matching can be identified with the set of edges $e$ with $x(e)=1$, being a union of pairwise vertex-disjoint circuits and simple paths.

Now Edmonds [1965d] showed the following min-max relation for simple 2-matchings.

Theorem 13. Let $G=(V, E)$ be an undirected graph. The maximum size of a simple 2-matching is equal to

$$
\min _{V^{\prime} \subseteq V}|V|+\left|V^{\prime}\right|-\frac{1}{2}(|K|+\kappa),
$$

where $K$ is the set of vertices having degree at most one in $\left\langle V \backslash V^{\prime}\right\rangle$ and where $\kappa$ denotes the number of components of $\left\langle V \backslash V^{\prime}\right\rangle$ containing an odd number of vertices in $K$.

Proof. The theorem follows from formula (8) for the maximum size of an uncapacitated $b$-matching. Indeed, add for each edge $e=\{u, v\}$ of $G$ two new vertices $u_{e}$ and $v_{e}$, and replace $e$ by the new edges $\left\{u, u_{e}\right\},\left\{u_{e}, v_{e}\right\},\left\{v_{e}, v\right\}$. Define $b(v)=2$ if $v$ is an "old" vertex, and $b(v)=1$ if $v$ is a "new" vertex. Then the maximum size of a $b$-matching in the new graph is exactly $|E|$ more than the maximum size of a simple 2-matching in the original graph. Applying (8) to the new graph gives the theorem.

The construction described here occurs in Tutte [1954]. The theorem also presents a good characterization for the existence of a perfect simple 2-matching (cf. Belck [1950], Tutte [1952]). The construction given in this proof, together with the splitting technique of vertices for 2-matchings described before, also reduces algorithmically (in polynomial time) the maximum weighted simple 2-matching problem to the maximum weighted matching problem.

Similarly polyhedral results follow. E. g., the perfect simple 2-matching polytope (being the convex hull of the perfect simple 2-matchings) is determined by:

$$
\begin{array}{ll}
0 \leqslant x(e) \leqslant 1 & (e \in E), \\
x(\delta(v))=2 & (v \in V), \\
x\left(\delta\left(V^{\prime}\right)\right)+\left|E^{\prime}\right|-2 x\left(E^{\prime}\right) \geqslant 1 & \left(V^{\prime} \subseteq V, E^{\prime} \subseteq \delta\left(V^{\prime}\right),\left|E^{\prime}\right| \text { odd }\right) .
\end{array}
$$


General capacitated $b$-matchings can be treated similarly. Given an undirected graph $G=(V, E)$, and functions $b: V \rightarrow \mathbb{Z}_{+}$and $c: E \rightarrow \mathbb{Z}_{+}$, one derives a min-max relation for the maximum size or weight of a $b$-matching $x$ subject to $c$ (i. e., $x \leqslant c$ ) again with Tutte's construction. Replace any edge $e=\{u, v\}$ by a path $\left\{u, u_{e}\right\},\left\{u_{e}, v_{e}\right\},\left\{v_{e}, v\right\}$, and define $\tilde{b}(v)=b(v)$ for the old vertices $v$, and $\tilde{b}\left(u_{c}\right)=\tilde{b}\left(v_{e}\right)=c(e)$ for the new vertices. Then the maximum size of a $\tilde{b}$-matching in the new graph is exactly $c(E)$ more than the maximum size of a $b$-matching subject to $c$ in the original graph. Applying (8) gives a min-max relation for capacitated $b$-matching.

Similarly, a good characterization for the existence of perfect capacitated $b$ matchings, the weighted case, and the polynomial solvability can be reduced to the uncapacitated case (see Belck [1950], Tutte [1952, 1954, 1981], Edmonds and Johnson [1973], Marsh [1979]).

In this way it follows that the convex hull of the $b$-matchings subject to $c$ is given by:

$$
\begin{array}{ll}
0 \leqslant x(e) \leqslant c(e) & (e \in E), \\
x(\delta(v)) \leqslant b(v) & (v \in V), \\
x\left(\left\langle V^{\prime}\right\rangle\right)+x\left(E^{\prime}\right) \leqslant\left\lfloor\frac{1}{2}\left(b\left(V^{\prime}\right)+c\left(E^{\prime}\right)\right)\right\rfloor & \left(V^{\prime} \subseteq V, E^{\prime} \subseteq \delta\left(V^{\prime}\right)\right),
\end{array}
$$

and moreover, that this system is totally dual integral.

Similarly, or alternatively from (15), one has that the convex hull of the perfect $b$-matchings subject to $c$ is given by:

$$
\begin{array}{ll}
0 \leqslant x(e) \leqslant c(e) & (e \in E), \\
x(\delta(v))=b(v) & (v \in V), \\
x\left(\left\langle V^{\prime}\right\rangle\right)+c\left(E^{\prime}\right)-2 x\left(E^{\prime}\right) \geqslant 1 & \left(V^{\prime} \subseteq V, E^{\prime} \subseteq \delta\left(V^{\prime}\right), b\left(V^{\prime}\right)+c\left(E^{\prime}\right) \text { odd }\right) .
\end{array}
$$

Padberg and Rao [1982] showed that the constraints (16) can be checked in polynomial time. Hence the ellipsoid method also gives the polynomial solvability of weighted capacitated $b$-matching problems.

Lower and upper bounds. Also lower bounds can be fetched into the min-max framework. Let $G=(V, E)$ be an undirected graph, and let $b_{1}, b_{2}: V \rightarrow \mathbb{Z}_{+}$. Then the convex hull of the functions $x: E \rightarrow \mathbb{Z}_{+}$satisfying

$$
b_{1}(v) \leqslant x(\delta(v)) \leqslant b_{2}(v) \quad(v \in V)
$$

is given by the constraints

$$
\begin{aligned}
& \text { (18) } \begin{array}{cc}
\text { (i) } x(e) \geqslant 0 & (e \in E), \\
\text { (ii) } b_{1}(v) \leqslant x(\delta(v)) \leqslant b_{2}(v) & (v \in V), \\
\text { (iii) } x\left(\left\langle V^{\prime}\right\rangle\right)-x\left(\left\langle V^{\prime \prime}\right\rangle\right)-x\left(\delta\left(V^{\prime \prime}\right) \backslash \delta\left(V^{\prime}\right)\right) \leqslant\left\lfloor\frac{1}{2}\left(b_{2}\left(V^{\prime}\right)-b_{1}\left(V^{\prime \prime}\right)\right)\right\rfloor
\end{array} \\
&
\end{aligned}
$$

(cf. Schrijver and Seymour [1977]). 
This may be reduced to capacitated $b$-matchings as follows. Let for each vertex $v$ of $G, v^{\prime}$ be a new vertex, and add to $G$ the new edges $\left\{v, v^{\prime}\right\}$ for $v \in V$, and $\left\{u^{\prime}, v^{\prime}\right\}$ for all $u, v \in V$. Let $b(v)=b\left(v^{\prime}\right)=b_{2}(v)$ for $v \in V$, and let $c\left(\left\{v, v^{\prime}\right\}\right)=b_{2}(v)-b_{1}(v)$ for $v \in V$, and $c(e)=\infty$ (or very large) for all other edges of the new graph. Then $x: E \rightarrow \mathbb{Z}_{+}$satisfies (17), if and only if it is a projection of a perfect $b$-matching subject to $c$ in the extended graph. In this way (18) follows from (15).

Similarly good characterizations, the polynomial solvability, and the total dual integrality of (18) follows.

We can add capacities on the edges: like in the case of capacitated $b$-matchings we can dispose of them by splitting edges to paths of length three. This construction gives a good characterization of Lovász [1970] for the existence of subgraphs with valencies obeying certain prescribed lower and upper bounds. This also implies a min-max relation for the maximum "weight" $\sum_{e} w(e) x(e)$
of a (not-necessarily nonnegative) function $x: E \rightarrow \mathbb{Z}$ satisfying

$$
\begin{array}{ll}
c_{1}(e) \leqslant x(e) \leqslant c_{2}(e) & (e \in E), \\
b_{1}(v) \leqslant x(\delta(v)) \leqslant b_{2}(v) & (v \in V),
\end{array}
$$

where $c_{1}, c_{2}: E \rightarrow \mathbb{Z}$ and $b_{1}, b_{2}: V \rightarrow \mathbb{Z}$. Indeed, this can be reduced to the case $c_{1}=0$, as replacing $c_{1}(e)$ by $0, c_{2}(e)$ by $c_{2}(e)-c_{1}(e), b_{1}(v)$ by $\max \left\{0, b_{1}(v)-c_{1}(\delta(v))\right\}$ and $b_{2}(v)$ by $\max \left\{0, b_{2}(v)-c_{1}(\delta(v))\right\}$, does not change the essence of the problem.

This last problem is the undirected analogue of the general transshipment problem (Section 5, (10)). In fact, Edmonds [1967] and Edmonds and Johnson [1973] mixed the directed and undirected case to one problem on "bidirected flows". Let $A$ be a $\{0, \pm 1\}$-matrix, where each column has exactly two nonzero entries. Then for all vectors $c_{1}, c_{2}, b_{1}, b_{2}, w$ the integer LP-problem:

$$
\begin{array}{ll}
\operatorname{maximize} & w x \\
\text { subject to } & c_{1} \leqslant x \leqslant c_{2}, \\
& b_{1} \leqslant A x \leqslant b_{2}, \\
& x \text { integer, }
\end{array}
$$

has a good characterization, and can in fact be solved in polynomial time.

Indeed, the matrix $A$ can be considered as the incidence matrix of a "mixed" graph, where each edge has either two heads, or two tails, or a head and a tail. Clearly, if $A$ has no entries -1, (20) reduces to (19), while if each column of $A$ has both a +1 and a -1 , we have (10) of Section 5 .

Problem (20) can be reduced to (19) as follows (cf. Edmonds [1967]). Let $A=\left(a_{i j}\right)$ have $n$ rows and $m$ columns. Make an undirected graph with vertices $v_{1}^{-}, \ldots, v_{n}^{-}, v_{1}^{+}, \ldots, v_{n}^{+}$, and edges $e_{1}, \ldots, e_{m}$ with $v_{i}^{ \pm} \in e_{j}$ iff $a_{i j}= \pm 1$, (for $i=1, \ldots, n ; j=1, \ldots, n ; \pm=+,-$ ), and moreover edges $\left\{v_{i}^{-}, v_{i}^{+}\right\}$(for $i=1$, $\ldots, n)$. Define

$$
\begin{aligned}
& \tilde{b}_{1}\left(v_{i}^{+}\right):=\left(b_{1}\right)_{i} \text { and } \tilde{b}_{2}\left(v_{i}^{+}\right):=\left(b_{2}\right)_{i} \\
& \tilde{b}_{1}\left(v_{i}^{-}\right):=\tilde{b}_{2}\left(v_{i}^{-}\right):=0 \\
& (i=1, \ldots, n), \\
& (i=1, \ldots, n) \text {, }
\end{aligned}
$$




$$
\begin{array}{ll}
\tilde{c}_{1}\left(e_{j}\right):=\left(c_{1}\right)_{j} \text { and } \tilde{c}_{2}\left(e_{j}\right):=\left(c_{2}\right)_{j} & (j=1, \ldots, m), \\
\tilde{c}_{1}\left(\left\{v_{i}^{-}, v_{i}^{+}\right\}\right):=-\infty \text { and } \tilde{c}_{2}\left(\left\{v_{i}^{-}, v_{i}^{+}\right\}\right):=\infty & (i=1, \ldots, n) .
\end{array}
$$

Then problem (20) is equivalent to finding a maximum weighted integer solution for (19) (after adding ). In this way a good characterization and the polynomial solvability follows. Note that this construction generalizes the one described in Remark 1 of Section 5, reducing network flow problems to bipartite matching.

$T$-joins and $T$-cuts. We are not yet at the end of our self-refining trip. It turns out that also certain parity constraints can be added. We first consider the case of $T$-joins and $T$-cuts.

Let $G=(V, E)$ be an undirected graph, and let $T \subseteq V$ be such that $|T|$ is even. A $T$-join is a set $E^{\prime}$ of edges such that $T$ is exactly the set of vertices in the graph $\left(V, E^{\prime}\right)$ of odd valency. A $T$-cut is a set of edges of the form $\delta\left(V^{\prime}\right)$ with $V^{\prime} \subseteq V$ and $\left|V^{\prime} \cap T\right|$ odd. It is immediate that each $T$-join intersects each $T$-cut. In fact, the minimal $T$-joins are exactly the minimal sets of edges intersecting all $T$ cuts, and vice versa (minimal under inclusion). Note that minimal $T$-joins do not contain circuits, and always form the edge-disjoint union of a collection of $\frac{1}{2}|T|$ paths, containing each vertex in $T$ exactly once as an end point.

Edmonds and Johnson [1973] proved the following.

Theorem 14. The minimum size of a T-join is equal to half of the maximum number $t$ of $T$-cuts $C_{1}, \ldots, C_{t}$ (repetition allowed) such that no edge is contained in more than two of the $C_{i}$.

Proof. This may be derived from the minimum weight perfect matching problem, and the total dual half-integrality of (6), as follows. Replace any edge $e=\{u, v\}$ of $G$ by the new edge $e^{\prime}=\left\{u_{e}, v_{e}\right\}$, where the new edges are pairwise disjoint. Next, for each $v$ in $V$, add edges $\left\{v_{e}, v_{f}\right\}$ for all pairs of edges $e, f$ containing $v$. Moreover, if $v \in T$ and has even degree, or if $v \notin T$ and has odd degree, add a new vertex $v_{0}$, and edges $\left\{v_{0}, v_{e}\right\}$ for all edges $e$ containing $v$. So vertices $v$ in $T$ correspond to a clique of odd size in the new graph, and the other vertices to a clique of even size.

Define weights as follows. For $e$ in $E, w\left(e^{\prime}\right):=1$, and for the other edges the weight is 0 . Then the minimum weight of a perfect matching in the new graph is equal to the minimum size of a $T$-join in $G$, and (6) applies.

Similarly, the weighted case, and the algorithmic problem can be reduced to perfect matchings. Note that Theorem 14 itself is of a self-refining nature: the weighted case follows by replacing each edge $e$ by a path of length equal to its weight (with the same end points as $e$ ).

It similarly follows that the convex hull of the subsets of $E$ containing a $T$-join (i. e., intersecting all $T$-cuts) is determined by:

$$
\begin{array}{ll}
0 \leqslant x(e) \leqslant 1 & (e \in E), \\
x\left(E^{\prime}\right) \geqslant 1 & \left(E^{\prime} T \text {-cut }\right),
\end{array}
$$

and that (22) is totally dual half-integral. 
In general (22) is not totally dual integral, as is shown by taking $G=K_{4}$ and $T=V$. This is essentially the only counterexample, as Seymour [1977] showed that (22) is totally dual integral, if and only if $V$ cannot be partitioned into subsets $V_{1}, V_{2}, V_{3}, V_{4}$, each intersecting $T$ in an odd number of vertices, such that each $V_{i}$ and each union of any two of the $V_{i}$ induce a connected subgraph of $G$. Besides, Seymour [1979a] showed that if $G$ is bipartite then the minimum size of a $T$-join is equal to the maximum number of pairwise disjoint $T$-cuts. This strengthens Theorem 14, and actually implies it as a special case.

Theorem 14 contains the following special cases. If $|T|=2$, minimal $T$-joins are exactly minimal paths. If $T=V$, and we add a large number to all weights, the minimum weight $T$-join problem is equivalent to the minimum weight perfect matching problem. If we take $T$ to be the set of all vertices of odd degree in $G, T$-joins are exactly those sets of edges which make $G$ eulerian by "doubling" them. That is, the minimum weight of a $T$-join is equal to the minimum weight of edges which should be traversed twice in order to make a tour through the graph traversing all edges at least once. Thus, it gives a min-max relation for the Chinese postman problem.

By the theory of blocking polyhedra (cf. Section 3), the characterization (22) implies that the convex hull of the sets of edges containing a $T$-cut (i.e., intersecting all $T$-joins) is determined by

$$
\begin{array}{ll}
0 \leqslant x(e) \leqslant 1 & (e \in E) \\
x\left(E^{\prime}\right) \geqslant 1 & \left(E^{\prime} T \text {-join }\right) .
\end{array}
$$

So this yields a min-max relation for the minimum capacity of a $T$-cut, which contains, e.g., for $|T|=2$ the fractional, undirected version of the max-flow min-cut theorem. Padberg and Rao [1982] showed that minimum-capacitated $T$-cuts can be found in polynomial time.

In general, (23) is not totally dual integral, even not totally dual half integral (cf. Seymour [1979a]). Seymour [1977] characterized pairs of graphs $G=(V, E)$ and subsets $T$ of $V$ for which (23) is totally dual integral.

Note that the construction given in the proof of Theorem 14 above also works in the weighted case, even if negative weights occur. Thus, also if negative weights occur, minimum weight $T$-joins are well characterized. This may be derived alternatively from the case of nonnegative weights as follows. Let $G=(V, E)$ be an undirected graph, and let $T \subseteq V$ with $|T|$ even. Let $w: E \rightarrow \mathbb{R}$ and let $E_{0}$ be the set of edges of negative weight. Let $T_{0}$ be the set of vertices of odd degree in the graph $\left(V, E_{0}\right)$. Then a subset $E^{\prime}$ of $E$ is a $T$-join, if and only if $E^{\prime} \Delta E_{0}$ is a $T \Delta T_{0}$-join ( $\Delta$ denoting symmetric difference). Moreover, let $\tilde{w}(e):=|w(e)|$ for $e$ in $E$. Then $\tilde{w}\left(E^{\prime}\right)=w\left(E^{\prime} \Delta E_{0}\right)-w\left(E_{0}\right)$, and hence finding a $T$ join $E^{\prime}$ with $w\left(E^{\prime}\right)$ minimal, is equivalent to finding a $T \Delta T_{0}$-join $E^{\prime \prime}$ with $\tilde{w}\left(E^{\prime \prime}\right)$ minimal.

So in case $|T|=2$ we obtain a min-max relation and a polynomial algorithm for shortest paths in undirected graphs, where lengths may be negative, provided that all circuits have nonnegative length (cf. Remark 3 in Section 5). 
One similarly derives that the convex hull of the $T$-joins is given by:

$$
\begin{array}{ll}
0 \leqslant x(e) \leqslant 1 & (e \in E), \\
x\left(\delta\left(V^{\prime}\right)\right)+\left|E^{\prime}\right|-2 x\left(E^{\prime}\right) \geqslant 1 & \left(V^{\prime} \subseteq V, E^{\prime} \subseteq \delta\left(V^{\prime}\right),\left|V^{\prime} \cap T\right|+\left|E^{\prime}\right| \text { odd }\right) .
\end{array}
$$

In case $T=\emptyset$ we obtain the convex hull of eulerian subgraphs.

Finally, by combining constructions like those describe in this section, one may derive a min-max relation, and a polynomial algorithm, for the following more general problem:

$$
\begin{array}{lll}
\operatorname{maximize} & \sum_{e \in E} w(e) x(e), & \\
\text { such that } & c_{1}(e) \leqslant x(e) \leqslant c_{2}(e) & (e \in E), \\
& b_{1}(v) \leqslant x(\delta(v)) \leqslant b_{2}(v) & (v \in V), \\
& x(\delta(v)) \equiv 0(\bmod 2) & (v \in S), \\
& x(\delta(v)) \equiv 1(\bmod 2) & (v \in T), \\
& x(e) \text { integer } & (e \in E) .
\end{array}
$$

where $G=(V, E)$ is an undirected graph, $T$ and $S$ are disjoint subsets of $V$, and $w, c_{1}, c_{2}: E \rightarrow \mathbb{Z} \cup\{ \pm \infty\}$ and $b_{1}, b_{2}: V \rightarrow \mathbb{Z} \cup\{ \pm \infty\}$.

More generally, one derives similarly from the weighted matching problem a min-max relation for the integer LP-problem

$$
\begin{aligned}
\operatorname{maximize} & w x \\
\text { subject to } & c_{1} \leqslant x \leqslant c_{2}, \\
& b_{1} \leqslant A x \leqslant b_{2}, \\
& x \text { integer, }
\end{aligned}
$$

where $A$ is a matrix with entries from $\{0, \pm 1, \pm 2\}$, such that the sum of the absolute values of the entries in any column is at most 2 , and where $w, c_{1}, c_{2}, b_{1}, b_{2}$ are arbitrary vectors (of size compatible for (26)) - see Edmonds and Johnson $[1970,1973]$. Similarly, the polynomial solvability of (26) follows. Problem (26) contains most of the problems treated before.

A min-max relation for (26) can be described as follows. Without loss of generality let $c_{1}, c_{2}, b_{1}, b_{2}$ be integer. Denote the inequality system given in (26), without the integrality constraint, by $C x \leqslant d$. Then if we add to $C x \leqslant d$ the inequalities

$$
y C \leqslant\lfloor y d\rfloor \quad\left(y\left\{0, \frac{1}{2}\right\} \text {-vector with } y C \text { integer }\right),
$$

we obtain the convex hull of the integer solutions of $C x \leqslant d$, and we could leave out the integrality constraint. Adding (27) makes the system totally dual integral.

It is an open problem to characterize the matrices $A$ enjoying this property (i. e., that for all $c_{1}, c_{2}, b_{1}, b_{2}$, the addition of (27) makes the system $c_{1} \leqslant x \leqslant c_{2}$, $b_{1} \leqslant A x \leqslant b_{2}$ totally dual integral). Beside the matrices given above, also for any 
totally unimodular matrix $B$, the matrices $A=B$ and $A=2 B$ have this property. It is easy to see that any matrix with this property has entries only $0, \pm 1, \pm 2$.

\section{Multicommodity Flows}

In Section 5 we studied network flows linking one "commodity", i. e., linking one pair of source and sink. Often, however, one is interested in multicommodity flows, linking several pairs of sources and sinks simultaneously (like in telephone networks). In general, such problems turned out to be hard (NP-complete), and no min-max relations could be derived. However, in some special cases polynomial algorithms and min-max relations have been found. These special cases all concern undirected graphs.

Here we represent a multicommodity flow as a packing of paths (which is for our purposes equivalent to the usual representation of multicommodity flows). Let $G=(V, E)$ be an undirected graph, and let $\left\{r_{1}, s_{1}\right\}, \ldots,\left\{r_{k}, s_{k}\right\}$ be different pairs of vertices of $G$. These pairs are called the commodities. Then a multicommodity flow is given by paths $P_{1}, \ldots, P_{s}$ and nonnegative numbers $\lambda_{1}, \ldots, \lambda_{s}$ such that, for $j=1, \ldots, s, P_{j}$ is an $r_{i}-s_{i}$-path for some $i=1, \ldots, k$. Its $i$-th value is equal to the sum of those $\lambda_{j}$ for which $P_{j}$ is an $r_{i}-s_{i}$-path. The total value is the sum of these values, which equals $\lambda_{1}+\ldots+\lambda_{s}$. If a capacity function $c: E \rightarrow \mathbb{R}_{+}$is given, the multicommodity flow is said to be subject to $c$ if for each edge $e$ of $G$ the sum of those $\lambda_{j}$ for which $P_{j}$ contains $e$ is at most $c(e)$.

A multicommodity cut is a set of edges separating each of the pairs $\left\{r_{1}, s_{1}\right\}, \ldots,\left\{r_{k}, s_{k}\right\}$.

Now the following inequality always holds:

(1) given an undirected graph $G=(V, E)$, commodities $\left\{r_{1}, s_{1}\right\}, \ldots,\left\{r_{k}, s_{k}\right\}$, and a capacity function $c: E \rightarrow \mathbb{R}_{+}$, the maximum total value of a multicommodity flow subject to $c$, is not more than the minimum capacity of a multicommodity cut.

If $k=1$ we have equality by the max-flow min-cut theorem. The graph $K_{1,3}$ (with commodities all pairs of vertices of valency one) shows that in general one does not have equality in (1). However, equality has been proved in the following special cases.

I. Two commodities. If there are only two commodities, (1) holds with equality. This is the content of Hu's two-commodity flow theorem [1963]. Hu also gave a polynomial algorithm finding an optimum multicommodity flow, thereby also showing that if the capacities are integer, there exists a half-integer optimum multicommodity flow (i.e., with the $\lambda_{j}$ half-integer).

Rothschild and Whinston [1966a, 1966b] (cf. Lovász [1976b], Seymour [1979b]) showed that if $G=(V, E)$ is eulerian (i.e., all valencies are even), and $r_{1}, s_{1}, r_{2}, s_{2} \in V$, then the maximum number of pairwise edge-disjoint paths, 
each connecting either $r_{1}$ and $s_{1}$ or $r_{2}$ and $s_{2}$, is equal to the minimum size of a cut separating both $r_{1}$ and $s_{1}$, and $r_{2}$ and $s_{2}$. It is not difficult to see that this generalizes Hu's result.

Seymour [1977] characterized undirected graphs $G=(V, E)$ and commodities $\left\{r_{1}, s_{1}\right\},\left\{r_{2}, s_{2}\right\}$ for which there exists an integer optimum two-commodity flow for each integer capacity function on $E$. In general, finding an optimum integer two-commodity flow is NP-complete.

II. Commodities with a common source. If $r_{1}=\ldots=r_{k}$, equality in (1) can be derived from the max-flow min-cut theorem: add a new vertex $s$, and add edges $\left\{s_{1}, s\right\}, \ldots,\left\{s_{k}, s\right\}$, with high capacity. It follows moreover that if all capacities are integer, there is an integer optimum multicommodity flow.

III. Kleitman, Martin-Löf, Rothschild and Whinston theorem. If the commodities form a union of complete bipartite graph, each two of which cover $V$, then (1) holds with equality. If all capacities are integer, there exists an integer optimum flow. This was proved by Kleitman, Martin-Löf, Rothschild and Whinston [1970].

In particular, in each of the cases I-III the convex hull of the multicommodity cuts is given by:

$$
\begin{array}{ll}
0 \leqslant x(e) \leqslant 1 & (e \in E), \\
x\left(E^{\prime}\right) \geqslant 1 & \left(E^{\prime} r_{i}-s_{i} \text {-path for some } i=1, \ldots, k\right) .
\end{array}
$$

Actually, this system is totally dual half-integer for two commodities (while Seymour [1977] characterized when (2) is totally dual integral), and totally dual integral in cases II and III.

Remark 4. Beside the cases I-III, in some more general cases the following good characterization holds:

(3) given an undirected graph $G=(V, E)$, commodities $\left\{r_{1}, s_{1}\right\}, \ldots,\left\{r_{k}, s_{k}\right\}$, a capacity function $c: E \rightarrow \mathbb{R}_{+}$, and "requirements" $\rho_{1}, \ldots, \rho_{k}$, then there exists a multicommodity flow with $i$-th value at least $\rho_{i}(i=1, \ldots, k)$, if and only if for all $V^{\prime} \subseteq V$, the capacity of $\delta\left(V^{\prime}\right)$ is not less than the sum of the $\rho_{i}$ for which $r_{i}$ and $s_{i}$ are separated by $V^{\prime}$.

This statement is true in the following cases:

(i) $\left|\left\{r_{1}, s_{1}, \ldots, r_{k}, s_{k}\right\}\right| \leqslant 4$ (Papernov [1976], Seymour [1980]);

(ii) there is a pair $\{u, v\}$ of vertices intersecting each of $\left\{r_{1}, s_{1}\right\}, \ldots,\left\{r_{k}, s_{k}\right\}$ (Papernov [1976]);

(iii) $\left\{r_{1}, s_{1}\right\}, \ldots,\left\{r_{k}, s_{k}\right\}$ form a circuit of length five (Lomonosov [1978]);

(iv) $G$ is planar, and $r_{1}, s_{1}, \ldots, r_{k}, s_{k}$ are on the boundary of the unbounded face (Okamura and Seymour [1981]); (v) the graph $\left(V, E \cup\left\{\left\{r_{1}, s_{1}\right\}, \ldots,\left\{r_{k}, s_{k}\right\}\right\}\right)$ is planar (Seymour $[1978 \mathrm{~b}$,
$1981 \mathrm{a}]$ ).

In each of these cases, if the capacities and requirements are integer, and if there exists a multicommodity flow satisfying the capacities and requirements, there exists a half-integer such flow. 
Note that the graph $K_{1,3}$ (with commodities all pairs of end vertices) shows that none of the cases (i)-(v) in general has equality in (1). For an interesting characterization, see Lomonosov [1982].

If the commodities form a complete graph, there is a min-max relation for the maximum total value of an integer multicommodity flow, which relation was found by Mader [1978 a], and which differs from the relation suggested by (1).

For an undirected graph $G=(V, E)$ and a subset $S$ of $V$, a path is called an $S$-path if it has two distinct vertices in $S$ as its end points.

Theorem 15 (Mader's theorem). Let $S=\left\{s_{1}, \ldots, s_{k}\right\}$. Then the maximum number of pairwise edge-disjoint $S$-paths is equal to

$$
\min \frac{1}{2}\left(\sum_{i=1}^{k}\left|\delta\left(S_{i}\right)\right|-\mathcal{O}^{\prime}\left(V \backslash\left(S_{1} \cup \ldots \cup S_{k}\right)\right)\right),
$$

where the minimum ranges over all collections of pairwise disjoint subsets $S_{1}, \ldots, S_{k}$ of $V$ with $s_{1} \in S_{1}, \ldots, s_{k} \in S_{k}$, and where $\mathcal{O}^{\prime}\left(V \backslash\left(S_{1} \cup \ldots \cup S_{k}\right)\right)$ denotes the number of components $C$ of the graph $\left\langle V \backslash\left(S_{1} \cup \ldots \cup S_{k}\right)\right\rangle$ with $|\delta(C)|$ odd.

So if $G$ is eulerian, the $\mathscr{O}^{\prime}$-term in (4) disappears - see Lovász [1976b].

Mader [1978 b] also gave a vertex-disjoint analogue: assuming (without loss of generality) that $S$ is a coclique, the maximum number of pairwise internally vertex-disjoint $S$-paths is equal to

$$
\min \left|V_{0}\right|+\sum_{i=1}^{t}\left\lfloor\frac{1}{2}\left|V_{i}\right|\right\rfloor
$$

where the minimum ranges over all subsets $V_{0}, V_{1}, \ldots, V_{t}$ of $V(t \geqslant 0)$ such that each S-path not intersecting $V_{0}$ contains at least two vertices in at least one of $V_{1}, \ldots, V_{t}$. This theorem contains the Tutte-Berge theorem (Theorem 11) as special case.

Finally, there is the "polar" problem of packing multicommodity cuts. By the theory of blocking polyhedra (cf. Section 3), from the characterization (2) it follows that, given an undirected graph $G=(V, E)$ and commodities $\left\{r_{1}, s_{1}\right\}, \ldots,\left\{r_{k}, s_{k}\right\}$, the convex hull of the subsets $E^{\prime}$ which connect at least one of the pairs $\left\{r_{i}, s_{i}\right\}$, is determined by:

$$
\begin{array}{ll}
0 \leqslant x(e) \leqslant 1 & (e \in E), \\
x\left(E^{\prime}\right) \geqslant 1 & \left(E^{\prime} \text { multicommodity cut }\right),
\end{array}
$$

if we are in one of the cases I-III above.

$\mathrm{Hu}$ [1973] showed that this is not true in the general case. Moreover, Seymour [1978 a] showed that in case there are only two commodities, (6) is totally dual half-integral. More generally, he showed: if $G=(V, E)$ is a bipartite graph and $r_{1}, s_{1}, r_{2}, s_{2}$ are vertices of $G$, then the minimum length of a path connecting either $r_{1}$ and $s_{1}$, or $r_{2}$ and $s_{2}$, is equal to the maximum number of pairwise disjoint edge sets, each meeting every $r_{1}-s_{1}$-path and every $r_{2}-s_{2}$-path.

Furthermore, Seymour [1977] characterized when (6) is totally dual integral for two commodities. 


\section{Arborescences and Directed Cuts}

In this section we describe min-max relations which are centered around the concepts of arborescence, directed cut, and strong connector, and which again turn out to generalize those for bipartite graphs and network flows.

Arborescences and rooted cuts. Let $D=(V, A)$ be a directed graph, and let $r \in V$. An $r$-arborescence is a set $A^{\prime}$ of arcs of $D$ forming a rooted directed spanning tree, with root $r$. That is, $A^{\prime}$ contains no circuit, and each vertex $s \neq r$ is entered by exactly one arc in $A^{\prime}$. A cut rooted at $r$, or an $r$-cut, is a set of arcs of the form $\delta^{-}\left(V^{\prime}\right)$, with $\emptyset \neq V^{\prime} \subseteq V \backslash r$.

It is immediate that each $r$-arborescence intersects each $r$-cut. Moreover, the minimal $r$-cuts are exactly the minimal sets intersecting all $r$-arborescences, and vice versa.

Fulkerson [1974] (cf. Edmonds [1967a]) proved the following min-max equation.

Theorem 16 (Fulkerson's optimum branching theorem). Let $D=(V, A)$ be a directed graph, let $r \in V$ and let $l: A \rightarrow \mathbb{Z}_{+}$be a length function. Then the minimum length of an $r$-arborescence is equal to the maximum number $t$ of $r$-cuts $C_{1}, \ldots, C_{t}$ (repetition allowed) such that no arc $a$ is in more than $l(a)$ of the $C_{i}$.

Before we prove the theorem, observe the following. Let $B$ be the matrix with columns indexed by $A$, and with rows the incidence vectors of all $r$-cuts. Then the theorem states that for any $l: A \rightarrow \mathbb{Z}_{+}$, the optima in

$$
\min \{l x \mid x \geqslant 0, B x \geqslant 1\}=\max \{|y| \mid y \geqslant 0, y B \leqslant l\}
$$

are attained by integer optimum solutions. By the theory of total dual integrality (cf. Section 3), it suffices to show that the maximum in (1) has an integer optimum solution.

Proof. Let $y$ be an optimum solution for the maximum in (1), such that

$$
\sum_{\emptyset \neq V^{\prime} \subseteq V \backslash r} y_{\delta}-\left(V^{\prime}\right) \cdot\left|V^{\prime}\right|^{2}
$$

is as large as possible (such a $y$ exists, by reason of compactness). Now let

$$
\mathscr{F}:=\left\{V^{\prime} \subseteq V \mid y_{\delta}-\left(V^{\prime}\right)>0\right\} \text {. }
$$

Then $\mathscr{F}$ is laminar, i.e., if $U, W \in \mathscr{F}$ then $U \subseteq W$ or $W \subseteq U$ or $U \cap W=\emptyset$. For suppose to the contrary that $U \nsubseteq W \nsubseteq U$ and $U \cap W \neq \emptyset$. Let $0<\varepsilon \leqslant \min \left\{y_{\delta-(U)}, y_{\delta}-(W)\right\}$. Let the vector $y^{\prime}$ be given by:

$$
\begin{array}{ll}
y_{\delta-(U)}^{\prime}:=y_{\delta}-(U)-\varepsilon, & y_{\delta-(W)}^{\prime}:=y_{\delta}-(W)-\varepsilon, \\
y_{\delta}^{\prime}-(U \cap W):=y_{\delta}-(U \cap W)+\varepsilon, & y_{\delta-(U \cup W)}^{\prime}:=y_{\delta}-(U \cup W)+\varepsilon,
\end{array}
$$

and let $y^{\prime}$ coincide with $y$ in the other coordinates. Then $y^{\prime} \geqslant 0, y^{\prime} B \leqslant y B$, and $\left|y^{\prime}\right|=|y|$, so $y^{\prime}$ again is an optimum solution in (1). However (2) is augmented, contradicting the maximality of (2). 
Now let $B_{0}$ be the submatrix of $B$ consisting of those rows of $B$ corresponding to sets in $\mathscr{F}$. Then $B_{0}$ is totally unimodular (in fact, it is the incidence matrix of some directed graph). This can be seen with Ghouila-Houri's characterization of total unimodularity (cf. Section 3 ) as follows. The collection $\mathscr{F}$ can be partitioned into "levels" $\mathscr{F}_{1}, \mathscr{F}_{2}, \ldots$, by defining $\mathscr{F}_{i}$ inductively as the collection of maximal sets (under inclusion) in $\mathscr{F} \backslash\left(\widetilde{\mathscr{F}_{1}} \cup \ldots \cup \widetilde{\mathscr{F}_{i-1}}\right.$ ). By the laminarity of $\mathscr{F}$, each $\mathscr{F}_{i}$ consists of pairwise disjoint sets. Now let $R_{1}$ be the set of rows of $B_{0}$ corresponding to a set in $\mathscr{F}_{1}, \mathscr{F}_{3}, \mathscr{F}_{5}, \ldots$, and let $R_{2}$ be the set of the other rows of $B_{0}$. Then it is easy to see that the sum of the rows in $R_{1}$, minus the sum of the rows in $R_{2}$, is a $\{0,+1,-1\}$-vector. As such a splitting can be made similarly for each submatrix of $B_{0}$, by Ghouila-Houri's result $B_{0}$ is totally unimodular.

Now we have:

$$
\max \left\{\left|y_{0}\right| \mid y_{0} \geqslant 0, y_{0} B_{0} \leqslant l\right\}=\max \{|y| \mid y \geqslant 0, y B \leqslant l\} .
$$

Indeed, the inequality $\leqslant$ is trivial (by extending $y_{0}$ with zeroes), while the inequality $\geqslant$ follows from the fact that the second maximum in (5) is attained by the vector $y$ above, which has zeroes outside of $B_{0}$.

Since $B_{0}$ is totally unimodular, the first maximum, and hence also the second maximum, has an integer optimum solution.

So although the constraints $x \geqslant 0, B x \leqslant 1$ generally are not totally unimodular, integer optimum solutions are shown by proving that in the optimum the active constraints can be chosen to be totally unimodular. This method of proof is an example of a general technique for deriving total dual integrality see Edmonds and Giles [1977] (or Section 11 below). It consists of showing first that the active constraints in the optimum can be chosen to be "nice" (e.g., "laminar" or "cross-free"), and next that nice constraint sets are totally unimodular, so that integer optimum solutions follow from Hoffman and Kruskal's theorem.

Fulkerson's optimum branching theorem can be equivalently stated as the system

$$
\begin{array}{ll}
0 \leqslant x(a) \leqslant 1 & (a \in A), \\
x\left(A^{\prime}\right) \geqslant 1 & \left(A^{\prime} r \text {-cut }\right),
\end{array}
$$

being totally dual integral. In particular, (6) determines the convex hull of the sets of edges containing an $r$-arborescence.

Edmonds [1967 a] and Fulkerson [1974] designed a polynomial algorithm for finding a shortest arborescence. The polynomial solvability also follows with the ellipsoid method, as (6) can be checked in polynomial time, although there are exponentially many $r$-cuts: considering a given $x \in \mathbb{R}_{+}^{A}$ as a capacity function, we can find an $r$-cut of minimum capacity (e.g., with Ford and Fulkerson's algorithm); if this minimum is less than 1 the $r$-cut yields a violated inequality in (6), and otherwise (6) is satisfied.

By the theory of blocking polyhedra (cf. Section 3) it follows that if we replace " $r$-cut" in (6) by " $r$-arborescence", we obtain the convex hull of sets of edges containing an $r$-cut. Also total dual integrality is maintained, as follows 
from the following theorem of Edmonds [1973] (see Lovász [1976a] for a short proof).

Theorem 17 (Edmonds' disjoint branching theorem). Let $D=(V, A)$ be a directed graph, and let $r \in V$. Then the maximum number of pairwise disjoint $r$-arborescences is equal to the minimum size of an $r$-cut.

The weighted case follows by replacing arcs by parallel arcs.

Note that the (easy) max-potential min-work theorem, and the max-flow min-cut theorem (Theorems 5 and 4) follow from Theorems 16 and 17, respectively, by adding arrows $(s, v)$ for each $v$ in $V$, of length zero or high capacity.

Giles [1982 a, 1982b, 1982 c] considered matching forests, which generalize matchings and arborescences.

Directed cuts and their coverings. Let $D=(V, A)$ be a directed graph. A directed cut is a set of arcs of the form $\delta^{-}\left(V^{\prime}\right)$, where $\emptyset \neq V^{\prime} \neq V$ and $\delta^{+}\left(V^{\prime}\right)=\emptyset$. A (directed cut) covering is a set of arcs intersecting all directed cuts. It follows that a set $A^{\prime}$ of arcs is a covering, if and only if the contraction of the arcs in $A^{\prime}$ makes $D$ strongly connected.

By a method similar to that of proving Fulkerson's optimum branching theorem ("uncrossing" cuts), one can show that the system

$$
\begin{array}{ll}
0 \leqslant x(a) \leqslant 1 & (a \in A), \\
x\left(A^{\prime}\right) \geqslant 1 & \left(A^{\prime} \text { directed cut }\right),
\end{array}
$$

is totally dual integral. Hence the polyhedron determined by (7) has integer vertices, and is the convex hull of the directed cut coverings.

The total dual integrality of (7) is equivalent to the following theorem of Lucchesi and Younger [1978] (cf. Lovász [1976a]).

Theorem 18 (Lucchesi-Younger theorem). Let $D=(V, A)$ be a directed graph. Then the minimum size of a directed cut covering is equal to the maximum number of pairwise disjoint directed cuts.

The weighted version follows by replacing arcs by directed path. If $D$ is a complete bipartite graph, with all arcs directed from one colour class to the other, this weighted version is equivalent to the weighted version of König's covering theorem ((8) in Section 4).

Since the system (7) can be checked in polynomial time (as it amounts to finding a directed cut of minimum capacity (taking $x$ as capacity), which can be done with Ford and Fulkerson's minimum cut algorithm), also minimum length directed cut coverings can be found in polynomial time, with ellipsoids. For direct algorithms, see Karzanov [1979], Lucchesi [1976], Frank [1981].

It is conjectured by Edmonds and Giles [1977] that also the "polar" minmax relation holds, i. e., that in any directed graph, the minimum size of a directed cut is equal to the maximum number of pairwise disjoint directed cut coverings. This conjecture is still unsettled. However, in Schrijver [1980] a counterexample to the weighted version is given. So the system

$$
\begin{array}{ll}
0 \leqslant x(a) \leqslant 1 & (a \in A), \\
x\left(A^{\prime}\right) \geqslant 1 & \left(A^{\prime} \text { directed cut covering }\right)
\end{array}
$$


is in general not totally dual integral, although, by characterization (7) and the theory of blocking polyhedra (Section 3), (8) determines the convex hull of the sets of arcs containing a directed cut.

Feofiloff and Younger, and Schrijver [1982a] showed that Edmonds and Giles' conjecture, and its weighted version, are true in the case formulated in the following theorem.

Theorem 19. Let $D=(V, A)$ be an acyclic directed graph, such that each pair of source and sink is connected by a directed path. Let $c: A \rightarrow \mathbb{Z}_{+}$be a capacity function. Then the minimum capacity of a directed cut is equal to the maximum number $t$ of directed cut coverings $C_{1}, \ldots, C_{t}$ such that no arc a is in more than $c(a)$ of the $C_{i}$.

The weighted version is also true if $D$ comes from a directed tree by adding all $\operatorname{arcs}(u, v)$ for which there exists an $u$ - $v$-path. Moreover, the cardinality version is true if the minimum size of a directed cut is two (but not the weighted version).

Strong connectors. Let $D_{0}=\left(V, A_{0}\right)$ be a directed graph, and let $D=(V, A)$ be a second directed graph. A subset $A^{\prime}$ of $A$ is called a strong connector $\left(\right.$ for $\left.D_{0}\right)$ if the directed graph $\left(V, A_{0} \cup A^{\prime}\right)$ is strongly connected. A subset $A^{\prime}$ of $A$ is called a cut (induced by $D_{0}$ ) if $A^{\prime}=\delta_{A}^{-}\left(V^{\prime}\right)$ for some nonempty proper subset $V^{\prime}$ of $V$ with $\delta_{\bar{A}_{0}}\left(V^{\prime}\right)=\emptyset$.

It is easy to see that a set of $\operatorname{arcs}$ of $D$ is a strong connector, if and only if it intersects each cut induced by $D_{0}$. The following two theorem were shown in Schrijver [1982 a].

Theorem 20. Suppose $D_{0}$ is acyclic, and for all $(u, v)$ in $A$ there exist $u^{\prime}, v^{\prime}$ in $V$ and directed paths in $D_{0}$ from $u$ to $u^{\prime}$, from $v^{\prime}$ to $u^{\prime}$, and from $v^{\prime}$ to $v$. Let $l: A \rightarrow \mathbb{Z}_{A}$ be a length function. Then the minimum length of a strong connector for $D_{0}$ is equal to the maximum number $t$ of cuts induced by $D_{0}$ such that no arc a is in more than I(a) of these cuts.

Theorem 21. Suppose $D_{0}$ is acyclic, and each pair of source and sink of $D_{0}$ is connected by a directed path in $D_{0}$. Then the maximum number of pairwise disjoint strong connectors for $D_{0}$ is equal to the minimum size of a cut induced by $D_{0}$.

A capacitated version of Theorem 21 follows by replacing arcs by parallel arcs.

Theorems 20 and 21 contain the following special cases.

(i) If $r, s \in V$, and $A_{0}=\{(u, v) \mid u=s$ or $v=r\}$, then Theorem 20 is equivalent to the max-potential min-work theorem (Theorem 5), and Theorem 21 to Menger's theorem (Theorem 4).

(ii) If $V$ is the disjoint union of $V_{1}$ and $V_{2}, A_{0}=\left\{(u, v) \mid u \in V_{1}, v \in V_{2}\right\}$, and $A \subseteq\left\{(v, u) \mid u \in V_{1}, v \in V_{2}\right\}$, then Theorem 20 is equivalent to (the weighted version of) König's covering theorem (Corollary $2 \mathrm{a}$ and (8) in Section 4), while Theorem 21 is equivalent to Gupta's edge-colouring theorem (Corollary 3 a). 
(iii) If $r \in V$ and $A_{0}=\{(v, r) \mid v \in V\}$, then Theorem 20 is equivalent to Fulkerson's optimum branching theorem (Theorem 16), and Theorem 21 is equivalent to Edmonds' disjoint branching theorem (Theorem 17).

(iv) If $A \subseteq\left\{(u, v) \mid(v, u) \in A_{0}\right\}$, Theorem 20 is equivalent to the LucchesiYounger theorem (Theorem 18), while Theorem 21 is equivalent to Theorem 19.

Again, Theorem 20 can be shown by an uncrossing technique for cuts, like in the proof of Fulkerson's optimum branching theorem.

Note that the conditions for $D_{0}$ and $D$ given in Theorem 21 are more restrictive than those given in Theorem 20 .

We leave it to the reader to formulate these theorems in terms of polyhedra and total dual integrality. With the theory of blocking polyhedra it will follow from Theorem 20, that under the conditions of Theorem 20 a min-max relation for fractional packings of strong connectors holds.

The following result (Schrijver [1983 a]) generalizes Theorem 21 and part of Theorem 20 . Let $\mathscr{C}$ be a family of nonempty proper subsets of the finite set $V$ such that $V^{\prime} \cap V^{\prime \prime} \in \mathscr{C}$ and $V^{\prime} \cup V^{\prime \prime} \in \mathscr{C}$ whenever $V^{\prime}, V^{\prime \prime} \in \mathscr{C}$ and $V^{\prime} \cap V^{\prime \prime} \neq \emptyset$ and $V^{\prime} \cup V^{\prime \prime} \neq V$ (i.e., $\mathscr{C}$ is a crossing family). For any directed graph $D=(V, A)$, call a set $A^{\prime}$ of arcs a cut induced by $\mathscr{C}$ if $A^{\prime}=\delta_{A}^{-}\left(V^{\prime}\right)$ for some $V^{\prime}$ in $\mathscr{C}$. Call $A^{\prime}$ a covering for $\mathscr{C}$ if it intersects all cuts induced by $\mathscr{C}$, i. e., if $\delta_{A^{\prime}}^{-}\left(V^{\prime}\right) \neq \emptyset$ for all $V^{\prime} \in \mathscr{C}$. Now the following are equivalent:

(9) (i) for each directed graph $D=(V, A)$ the minimum size of a cut induced by $\mathscr{C}$ is equal to the maximum number of pairwise disjoint coverings for $\mathscr{C}$;

(ii) for each directed graph $D=(V, A)$ and for each length function $l: A \rightarrow \mathbb{Z}_{+}$, the minimum length of a covering for $\mathscr{B}$ is equal to the maximum number $t$ of cuts $C_{1}, \ldots, C_{t}$ induced by $\mathscr{B}$ such that no arc $a$ is in more than $l(a)$ of these cuts;

(iii) there are no $V_{1}, V_{2}, V_{3}, V_{4}, V_{5}$ in $\mathscr{C}$ such that: $V_{1} \subseteq V_{3} \subseteq V_{5}, V_{1} \subseteq V_{2}$, $V_{2} \cup V_{3}=V, V_{3} \cap V_{4}=\emptyset, V_{4} \subseteq V_{5}$.

As directed graphs may have parallel arcs, property (i) can be seen to be equivalent to its capacitated version.

\section{Perfect Graphs}

We now consider a class of undirected graphs which is defined by a min-maxrelation. This class of perfect graphs was introduced by Berge [1961, 1962]. What makes the class interesting is that it is closed under taking complementary graphs, so that one min-max relation implies another. This was conjectured by Berge and proved by Lovász. For surveys on perfect graphs, see Golumbic [1980], Berge and Chvátal [1982], Lovász [1981b]. 
Let $G=(V, E)$ be an undirected graph, and consider the following numbers:

(1) $\omega(G)=$ the clique number of $G=$ the maximum size of a clique [a clique is a set of pairwise adjacent vertices];

$\gamma(G)=$ the colouring number of $G=$ the minimum number of colours needed to colour the vertices of $G$ such that no two adjacent vertices have the same colour (i.e., the minimum number of cocliques needed to cover $V$ );

$\alpha(G)=$ the coclique number of $G=$ the maximum size of a coclique [a coclique is a set of pairwise nonadjacent vertices];

$\bar{\gamma}(G)=$ the clique cover number of $G=$ the minimum number of cliques needed to cover $V$.

The following relations are trivial: $\omega(G) \leqslant \gamma(G), \alpha(G) \leqslant \bar{\gamma}(G), \alpha(G)=\omega(\bar{G})$, and $\bar{\gamma}(G)=\gamma(\bar{G})$, where $\bar{G}$ denotes the complementary graph of $G$ (which has vertex set $V$, two vertices being adjacent in $\bar{G}$ iff they are nonadjacent in $G$ ). The circuit on five vertices shows that the inequalities can be strict. of $G$.

Now $G$ is called perfect if $\omega\left(G^{\prime}\right)=\gamma\left(G^{\prime}\right)$ for each induced subgraph $G^{\prime}$

Implicitly we have met several classes of perfect graphs: (i) bipartite graphs (trivially); (ii) complements of bipartite graphs (by König's covering theorem (Corollary 2a)); (iii) line graphs of bipartite graphs (by König's matching theorem (Theorem 2)); (iv) complements of line graphs of bipartite graphs (by König's edge-colouring theorem (Theorem 3 )); (v) comparability graphs (which, by definition, arise from a partially ordered set $(V, \leqslant)$, two vertices being adjacent iff they are comparable; the perfectness is easy - cf. Theorem 8); (vi) complements of comparability graphs (by Dilworth's theorem (Theorem 7)).

It was conjectured by Berge [1961, 1962] and proved by Lovász [1972] that the complement of each perfect graph is perfect again. This result, the perfect graph theorem, implies König's covering theorem (as (ii) are the complements of (i)), hence also König's matching theorem (by Gallai's theorem), and therefore also König's edge-colouring theorem (as (iv) are the complements of (iii)). Similarly it implies Dilworth's theorem.

More classes of perfect graphs will be discussed after the proof of the perfect graph theorem. We give a polyhedral proof of this theorem, due to Fulkerson [1972], Lovász [1972] and Chvátal [1975]. To this end, define for any undirected graph $G=(V, E)$, the clique polytope as the convex hull of the cliques in G. Clearly, any vector $x$ in the clique polytope satisfies:

$$
\begin{aligned}
\text { (i) } x(v) \geqslant 0 & (v \in V), \\
\text { (ii) } x(S) \leqslant 1 & (S \subseteq V, S \text { coclique }) .
\end{aligned}
$$

The circuit on five vertices shows that generally the polyhedron (2) can be larger than the clique polytope. Chvátal [1975] showed that the clique polytope coincides with (2) if and only if $G$ is perfect. This can be seen to imply the perfect graph theorem. 
First observe the following. Let $A x \leqslant 1$ denote the inequality system (2) (ii). So the rows of $A$ are the incidence vectors of cocliques. Then it follows from the definition of perfectness that $G$ is perfect if and only if the optima in

$$
\max \{w x \mid x \geqslant 0, A x \leqslant 1\}=\min \{|y| \mid y \geqslant 0, y A \geqslant w\}
$$

have integer optimum solutions, for each $\{0,1\}$-vector $w$.

Theorem 22. A graph $G$ is perfect if and only if its clique polytope is determined by (2).

Proof. I. First let $G$ be perfect. For $w: V \rightarrow \mathbb{Z}_{+}$, let $c_{w}$ denote the maximum weight of a clique. To prove that the clique polytope is given by (2), it suffices to show that

$$
c_{w}=\max \{w x \mid x \geqslant 0, A x \leqslant 1\}
$$

for each $w: V \rightarrow \mathbb{Z}_{+}$. This will be done by induction on $w(V)$.

If $w$ is a $\{0,1\}$-vector (4) follows from the note made on (3). So we may assume that $w(u) \geqslant 2$ for a certain vertex $u$. Let $e(u)=1$, and $e(v)=0$ if $v \neq u$. Replacing $w$ by $w-e$ in (3) and (4), gives by induction a vector $y \geqslant 0$ such that $y A \geqslant w-e$ and $|y|=c_{w-e}$. Since $(w-e)(u) \geqslant 1$, there is a coclique $S$ with $y_{S}>0$ and $u \in S$. Let $a$ be the incidence vector of $S$ (we may assume $a \leqslant w-e$ ).

Then $c_{w-a}<c_{w}$. For suppose $c_{w-a}=c_{w}$. Let $C$ be any clique with $(w-a) C=c_{w-a}$. Since $c_{w-a}=c_{w}, a(C)=0$. On the other hand, since $w-a \leqslant w-e \leqslant w$, we know that $(w-e) C=c_{w-e}$, and hence, by complementary slackness, $a(C)>0$.

Therefore

$$
c_{w}=1+c_{w-a}=1+\max \{(w-a) x \mid x \geqslant 0, A x \leqslant 1\} \geqslant \max \{w x \mid x \geqslant 0, A x \leqslant 1\},
$$

implying (4).

II. Conversely, suppose that the clique polytope of $G$ is determined by (2), i. e., that the maximum in (3) is attained by a clique for each $w$. To show that $G$ is perfect, it suffices to show that also the minimum in (3) has an integer optimum solution for each $w$ in $\{0,1\}^{V}$. This is shown by induction on $w(V)$.

Let $w \in\{0,1\}^{V}$, and let $y^{\prime}$ be a (not-necessarily integer) optimum solution for the minimum in (3). Let $S$ be a coclique with $y_{S}^{\prime}>0$, and let $a$ be its incidence vector (we may assume that $a \leqslant w$ ). Then the common value of

$$
\max \{(w-a) x \mid x \geqslant 0, A x \leqslant 1\}=\min \{|y| \mid y \geqslant 0, y A \geqslant w-a\}
$$

is less than the common value of (3), as decreasing component $y_{S}^{\prime}$ of $y^{\prime}$ a little bit, keeps it feasible in (6). However, the values in (3) and (6) are integer (as by assumption the maxima have integer optimum solutions). Hence they differ by exactly one. Moreover, by induction the minimum in (6) has an integer optimum solution $y^{*}$. Increasing component $y_{S}^{*}$ of $y^{*}$ by one, gives an integer optimum solution in (3).

The theory of anti-blocking polyhedra now gives directly the perfect graph theorem as a corollary. 
Corollary 22 a (perfect graph theorem). The complement of a perfect graph is perfect again.

Proof. If $G=(V, E)$ is perfect, by Theorem 22 the clique polytope $P$ is defined by (2). Hence, by the theory of anti-blocking polyhedra (cf. Section 3 ), the coclique polytope of $G$, i. e., the clique polytope of $\bar{G}$, is defined by (2) after replacing "coclique" by "clique", i. e., by "coclique for $\bar{G}$ ". Applying Theorem 22 again gives that $\bar{G}$ is perfect.

Stated otherwise, if $G$ is an undirected graph, then $\omega\left(G^{\prime}\right)=\gamma\left(G^{\prime}\right)$ for each induced subgraph $G^{\prime}$, if and only if $\alpha\left(G^{\prime}\right)=\bar{\gamma}\left(G^{\prime}\right)$ for each induced subgraph $G^{\prime}$.

The following classes of perfect graphs have been found.

(i) Bipartite graphs (König [1932]).

(ii) Line graphs of bipartite graphs (König [1916, 1931]).

(iii) Comparability graphs (Dilworth [1950]).

(iv) Chordal graphs (where each circuit of length at least four has a chord; Dirac [1961], Hajnal and Surányi [1958], Berge [1960]).

(v) Meyniel graphs (where each circuit of length odd and at least five has at least two chords; Meyniel [1976]).

(vi) Chvátal graphs (the edges of which can be oriented to an acyclic directed graph with no induced subgraph isomorphic to $(\{u, v, w, x\}$, $\{(u, v),(v, w),(x, w)\})$; Chvátal [1981]).

(vii) Unimodular graphs (where the incidence matrix of all maximal cliques form a totally unimodular matrix; Hoffman and Kruskal [1956]).

(viii) Cameron graphs (which arise from a directed graph $D=(V, A)$, and subset $T$ of $V$ intersecting each directed cycle in exactly one vertex, as follows: the vertex set is $V \backslash T$, two vertices being adjacent iff they occur together in some directed cycle of $D$; Cameron [1982] - cf. (11) in Section 5).

(ix) Edmonds-Giles graphs (which are graphs $G$ arising as follows: let $D=(V, A)$ be a directed graph, and let $\mathscr{C}$ be a collection of subsets of $V$, such that $\delta^{+}\left(V^{\prime}\right)=\varnothing$ for $V^{\prime} \in \mathscr{C}$, and such that if $V^{\prime}, V^{\prime \prime} \in \mathscr{C}$ with $V^{\prime} \cap V^{\prime \prime} \neq \varnothing$ and $V^{\prime} \cup V^{\prime \prime} \neq V$ then $V^{\prime} \cap V^{\prime \prime} \in \mathscr{C}$ and $V^{\prime} \cup V^{\prime \prime} \in \mathscr{C}$; let $G$ have vertex set $A$, two distinct arcs $a^{\prime}$ and $a^{\prime}$ of $D$ being adjacent in $G$ if and only if $a^{\prime}, a^{\prime \prime} \in \delta^{-}\left(V^{\prime}\right)$ for some $V^{\prime} \in \mathscr{C}$; Edmonds and Giles [1977] cf. Section 11).

(x) Complements of the graphs above.

We leave it to the reader to deduce the several inclusions between these classes.

It is conjectured by Berge [1969] that a graph is perfect if and only if it has no induced subgraph isomorphic to an odd circuit of odd length at least five or its complement. This strong perfect graph conjecture is still unsettled.

Suppose that we wish to derive, with the ellipsoid method, from the characterization (2) of the clique polytope, a polynomial algorithm determining a maximum clique in a perfect graph. Then we must be able to check the system (2) in polynomial time. However, this is equivalent to finding a maximum weighted coclique in $G$. This is the same as a maximum weighted clique in the 
complementary graph, which is perfect again. Hence, we cannot use (2) to obtain a polynomial algorithm for the clique problem for perfect graphs in general.

However, there is another, non-polyhedral min-max relation for $\omega(G)$. In Lovász [1979] it is shown that if $G$ is perfect, say with vertices $1, \ldots, n$, then:

$$
\begin{gathered}
\omega(G)=\max \left\{\begin{array}{r}
\sum_{i, j=1}^{n} b_{i, j} \mid B=\left(b_{i j}\right) \text { is a symmetric positive semi-definite } n \times n- \\
\text { matrix with trace } 1, \text { and with } b_{i j}=0 \text { if } i \neq j \text { and } i \text { and } \\
j \text { are not adjacent in } G\}=
\end{array}\right. \\
=\min \left\{\Lambda(C) \mid C=\left(c_{i j}\right) \text { is a symmetric } n \times n \text {-matrix, with } c_{i j}=1 \text { if } i=j\right. \\
\text { or } i \text { and } j \text { are adjacent in } G\},
\end{gathered}
$$

where $\Lambda(C)$ denotes the largest eigenvalue of $C$. Since the largest eigenvalue of a matrix can be approximated fast enough, (7) gives a good characterization for the clique problem for perfect graphs. Moreover, since the conditions in the maximum in (7) can be checked in polynomial time, the maximum itself, and hence $\omega(G)$ can be determined in polynomial time, again with ellipsoids. Similarly the weighted case can be handled - see Grötschel, Lovász and Schrijver [1981, 1981 a]. In Lovász [1981 b] it is shown that $G$ is perfect if and only if (7) holds for all induced subgraphs of $G$.

Other classes of graphs with a good characterization for $\omega(G)$ or $\alpha(G)$. Beside the perfect graphs there are some other classes of graphs for which the coclique or clique problem is well-characterized, or even polynomial solvable.

We saw already in Section 6 that the coclique number of the line graph of an undirected graph can be found in polynomial time, as this is the matching number of the original graph. (Clearly, also the clique number of a line graph can be computed in polynomial time.)

Minty [1980] and Sbihi [1978] showed more generally that in any claw-free graph, a maximum weighted coclique can be found in polynomial time. Here a graph is claw-free if it has no $K_{1,3}$ as an induced subgraph (e.g., line-graphs are claw-free). So this implies a good characterization for the (weighted) coclique number. However, no min-max relation for this number is known. Key problem here is characterizing (in terms of inequalities) the convex hull of the cocliques in a claw-free graph (cf. Giles and Trotter [1981]).

Other classes for which the weighted coclique number can be found in polynomial time are: circular arc graphs, circle arc graphs, and their complements (a circular arc graph (circle graph, respectively) has a collection of connected intervals (chords, respectively) of a circle as vertex set, two of them being adjacent iff they intersect (Gavril [1973, 1974])); graphs without "long" odd circuits (Hsu, Ikura and Nemhauser [1979]).

Another class of, not-necessarily perfect, graphs $G$ with $\omega(G)=\gamma(G)$ was found by Györi [1981]: let $A$ be a $\{0,1\}$-matrix, such that the ones in each row form a connected interval; then the graph $G$ has vertex set $\left\{(i, j) \mid a_{i j}=1\right\}$, where two pairs $(i, j)$ and $\left(i^{\prime}, j^{\prime}\right)$ are adjacent iff $a_{i j^{\prime}}=0$ or $a_{i^{\prime} j}=0$.

Finally, Sbihi and Uhry [1982] defined a graph $G=(V, E)$ to be $h$-perfect if its coclique polytope (the convex hull of the cocliques) is determined by: 


$$
\begin{array}{ll}
x(v) \geqslant 0 & (v \in V), \\
x(C) \leqslant 1 & (C \subseteq V, C \text { clique }), \\
x(C) \leqslant\left\lfloor\frac{1}{2}|C|\right\rfloor & (C \subseteq V, C \text { odd circuit }) .
\end{array}
$$

By Theorem 22, each perfect graph is $h$-perfect. Sbihi and Uhry described a constructive class of $h$-perfect graphs, including the "series-parallel graphs". Clearly, if $G$ is $h$-perfect, $\alpha(G)$ has a good characterization.

\section{Clutters and Blockers}

Many of the min-max relations dealt with above allow an interpretation in terms of "hypergraphs" or "clutters". Interesting theorems for these more general structures, extending these min-max results, were found by Fulkerson [1970, 1971, 1972], Lehman [1979], Lovász [1972, 1975, 1977], and Seymour [1977, 1979 a].

Let $S$ be a finite set. A collection $\mathscr{C}$ of subsets of $S$ is called a clutter if no two sets in $\mathscr{C}$ are contained in each other. The blocker $b(\mathscr{C})$ of $\mathscr{C}$ is the collection of minimal sets (under inclusion) intersecting all sets in $\mathscr{E}$. So $b(\mathscr{C})$ is a clutter again.

Edmonds and Fulkerson [1970] observed that for any clutter $\mathscr{E}$ the following duality phenomenon holds:

$$
b(b(\mathscr{C}))=\mathscr{C} .
$$

In the Sections $4-8$ we have met several examples of a clutter $\mathscr{E}$ together with its blocker $b(\mathscr{C})$ :

given:

(2) $G=(V, E)$

(3) $D=(V, A), r, s \in V$

(4) $G=(V, E), T \subseteq V$

(5) $G=(V, E), r_{1}, s_{1}, r_{2}, s_{2} \in V$ two-commodity cuts

(6) $D=(V, A), r \in V$

(7) $D=(V, A)$

(8) $D_{0}=\left(V, A_{0}\right), D=(V, A)$

$$
\mathscr{C} \text { contains the }
$$
minimal:

"stars"

$r$-s-cuts

$T$-cuts

$r$-cuts

directed cuts

cuts induced by $D_{0}$
$b(\mathbb{C})$ contains the

minimal:

edge coverings

$r$-s-paths

$T$-joins

$r_{1}-s_{1}$-paths and

$r_{2}-S_{2}$-paths

$r$-arborescences

directed cut coverings

strong connectors for $D_{0}$

where $G$ is an undirected graph, and $D$ and $D_{0}$ are directed graphs. A star is a collection of edges of the form $\delta(v)$ for some vertex $v$.

For any clutter $\mathscr{C}$ on a set $S$, let $M_{\mathscr{E}}$ denote the matrix with rows all incidence vectors of sets in $\mathscr{E}$. So the rows and columns of $M_{\mathscr{E}}$ are indexed by $\mathscr{E}$ and $S$, respectively. For $w: S \rightarrow \mathbb{Z}_{+}$consider the linear programming duality equation:

$$
\min \left\{w x \mid x \geqslant 0, M_{\mathscr{E}} x \geqslant 1\right\}=\max \left\{|y| \mid y \geqslant 0, y M_{\mathscr{E}} \leqslant w\right\} .
$$


One immediately sees that if the minimum has an integer optimum solution $x$, such a vector $x$ is the incidence vector of a set in $b(\mathscr{C})$.

Now $\mathscr{C}$ is said to have the $\mathbb{Q}_{+}$-max-flow min-cut property, or the $\mathbb{Q}_{+-}$ MFMC-property, if the minimum in (9) has an integer optimum solution (being the incidence vector of a set in $b(\mathscr{E})$ ), for each nonnegative vector $w$. Equivalently, if:

(10) $\left\{x \geqslant 0 \mid M_{\mathscr{E}} x \geqslant 1\right\}=\{x \mid x \geqslant z$ for some convex combination $z$ of the rows of $\left.M_{b(\mathbb{E})}\right\}$.

By the theory of blocking polyhedra (cf. Section 3), (10) is equivalent to the same equation with $\mathscr{C}$ and $b(\mathscr{C})$ interchanged:

(11) $\left\{x \geqslant 0 \mid M_{h(\mathscr{B})} x \geqslant 1\right\}=\{x \mid x \geqslant z$ for some convex combination $z$ of the rows of $\left.M_{\mathscr{E}}\right\}$.

Therefore, the following result of Lehman [1979] holds:

(12) $\mathscr{C}$ has the $\mathbb{Q}_{+}-M F M C$-property, if and only if $b(\mathscr{C})$ has the $\mathbb{Q}_{+}-M F M C$ property.

So if the minimum in (9) has an integer optimum solution for each nonnegative vector $w$, the same holds if we replace $\mathscr{C}$ by $b(\mathscr{C})$. Below we shall see that this gives several equivalences between combinatorial min-max relations.

First consider the following more general property. Let $\mathbb{K} \subseteq \mathbb{Q}_{+}$. The clutter $\mathscr{C}$ is said to have the $\mathbb{K}-M F M C$-property, if the minimum in (9) has an integer optimum solution $x$, and if the maximum has an optimum solution in $\mathbb{K}^{S}$, for each nonnegative integer vector $w$. In particular, $\mathscr{C}$ has the $\mathbb{Z}_{+}$-MFMC-property, if and only if the system $x \geqslant 0, M_{\mathscr{E}} x \geqslant 1$ is totally dual integral. Note that if $\mathbb{K}^{\prime} \supseteq \mathbb{K}$, then the $\mathbb{K}$-MFMC-property implies the $\mathbb{K}^{\prime}$-MFMC-property.

It is the content of König's (weighted) edge-covering theorem (Corollary $2 \mathrm{a}$ and (8) in Section 4), that for bipartite graphs the clutter $\mathscr{C}$ in (2) has the $\mathbb{Z}_{+}$MFMC-property. The fact that for bipartite graphs also $b(\mathscr{C})$ in $(2)$ has the $\mathbb{Z}_{+}$-MFMC-property is equivalent to Gupta's theorem (Corollary $3 \mathrm{a}$ ).

The clutters $\mathscr{E}$ and $b(\mathscr{C})$ in (3) both have the $\mathbb{Z}_{+}$-MFMC-property, which is the content of the max-potential min-work and the max-flow min-cut theorem (Theorem 5 and Corollary $4 \mathrm{~b}$ ), respectively.

The clutter $\mathscr{E}$ in (4) has the $\frac{1}{2} \mathbb{Z}_{+}$-MFMC-property (Theorem 14), hence $b(\mathscr{C})$ has the $\mathbb{Q}_{+}$-MFMC-property (and maybe the $\frac{1}{4} \mathbb{Z}_{+}$-MFMC-property, but generally not the $\frac{1}{2} \mathbb{Z}_{+}$-MFMC-property - see Seymour [1979 a]).

The clutters $\mathscr{C}$ and $b(\mathscr{C})$ in (5) both have the $\frac{1}{2} \mathbb{Z}_{+}$-MFMC-property, which was shown by Seymour [1978a] and Hu [1963], respectively - see Section 7.

The clutters $\mathscr{E}$ and $b(\mathscr{C})$ in (6) both have the $\mathbb{Z}_{+}$-MFMC-property, which is the content of Fulkerson's optimum branching theorem and Edmonds' disjoint branching theorem (Theorems 16 and 17), respectively.

The clutter $\mathscr{C}$ in (7) has the $\mathbb{Z}_{+}$-MFMC-property, by the Lucchesi-Younger theorem (Theorem 18), hence $b(\mathscr{C})$ has the $\mathbb{Q}_{+}$-MFMC-property. Generally, $b(\mathscr{C})$ does not have the $\mathbb{Z}_{+}$-MFMC-property (see Schrijver [1980]). It has this property under the conditions formulated in Theorem 19. 
The clutters $\mathscr{C}$ and $b(\mathscr{C})$ in (8) have the $\mathbb{Z}_{+}$-MFMC-property under the conditions formulated in Theorems 20 and 21, respectively.

Note that, roughly speaking, the ellipsoid method gives the following:

(13) if the clutter $\mathscr{C}$ on $S$ has the $\mathbb{Q}_{+}-M F M C$-property, then: there exists a polynomial algorithm finding a minimum weight set in $\mathscr{E}$, for each nonnegative weight function, if and only if there exists a polynomial algorithm finding a minimum weight set in $b(\mathscr{C})$, for each nonnegative weight function.

Indeed, if the minimum in (9) has an integer optimum solution, then a minimum weight set in $b(\mathscr{C})$ can be found in polynomial time, if the system $x \geqslant 0$, $M_{\mathscr{E}} x \geqslant 1$ can be checked in polynomial time. Considering $x$ as a weight function, this last amounts to finding a minimum weight set in $\mathscr{C}$.

In fact (13) could be stated more generally (and more precisely) in terms of classes of clutters, for which there exists a fixed polynomial algorithm finding minimum weight sets in the clutters (like Ford and Fulkerson's algorithm for all clutters $\mathscr{E}$ of $r$-s-cuts) - see Grötschel, Lovász and Schrijver [1981]. It is still an open question whether there exists a fixed polynomial algorithm finding minimum weight sets in any given clutter with the $\mathbb{Q}_{+}$-MFMC-property (which would make (13) trivial).

The problem of characterizing clutters with the $\mathbb{Z}_{+^{-}}, \frac{1}{2} \mathbb{Z}_{+-}$, or $\mathbb{Q}_{+}-$ MFMC-property is still unsettled for the greater part. Seymour [1977] showed that if $\mathscr{C}$ has the $\mathbb{K}$-MFMC-property, then any minor of $\mathscr{C}$ has the same property. Here a minor $\mathscr{C}$ ' arises from $\mathscr{C}$ by taking disjoint subsets $S_{1}$ and $S_{2}$ of $S$, and defining $\mathscr{C}^{\prime}$ to be the collection of minimal sets in

$$
\left\{S^{\prime} \backslash S_{1} \mid S^{\prime} \in \mathscr{E}, S^{\prime} \subseteq S \backslash S_{2}\right\} .
$$

So finding all minimal clutters (under taking minors) not having the $\mathbb{K}$ MFMC-property would characterize this property. As Seymour pointed out, this may be a difficult problem.

Seymour showed that the clutter $Q_{6}$, defined by

$$
Q_{6}:=\{\{1,3,5\},\{1,4,6\},\{2,3,6\},\{2,4,5\}\}
$$

(which could be identified with the triangles in the complete graph $K_{4}$ ), is a minimal clutter without the $\mathbb{Z}_{+}$-MFMC-property. However, $Q_{6}$ has the $\mathbb{Q}_{+-}$ MFMC-property, and $b\left(Q_{6}\right)$ has the $\mathbb{Z}_{+}$-MFMC-property. It was conjectured that if $\mathscr{E}$ has no $Q_{6}$ minor, and if $b(\mathscr{C})$ has the $\mathbb{Z}_{+}$-MFMC-property, then $\mathscr{C}$ has the $\mathbb{Z}_{+}$-MFMC-property. This is, however, contradicted by the example in Schrijver [1980].

Note that $Q_{6}$, and the clutters in (7), show that the $\mathbb{Z}_{+}$-MFMC-property is not invariant under taking blockers. Seymour [1978 a] gave an example showing that also the $\frac{1}{2} \mathbb{Z}_{+}$-property is not invariant under taking blockers.

Seymour [1977] was able to characterize the $\mathbb{Z}_{+}$-MFMC-property for an interesting class of clutters. A clutter $\mathscr{E}$ on $S$ is called binary if for all $S_{1}, \ldots, S_{k}$ in $\mathscr{C}$ with $k$ odd, the set $S_{1} \Delta \ldots \Delta S_{k}$ includes a set in $\mathscr{C}$. It is not difficult to prove that the blocker of a binary clutter is binary again. 
Examples of binary clutters are those of $r$-s-paths, $r$-s-cuts, $T$-joins, $T$-cuts, two-commodity paths and two-commodity cuts ((3), (4), (5) above).

Now Seymour proved:

Theorem 23. A binary clutter has the $\mathbb{Z}_{+}-M F M C$-property, if and only if it has no $Q_{6}$ minor.

Applied to the examples mentioned above, this theorem characterizes when the $\mathbb{Z}_{+}$-MFMC-property holds, i. e., when the system $x \geqslant 0, M_{\mathscr{C}} x \leqslant 1$ is totally dual integral. Another class of binary clutters is obtained as follows: let $G=(V, E)$ be an undirected graph, let $V$ be split into classes $R, S, R^{\prime}, S^{\prime}$, and let the clutter $\mathscr{C}$ consist of all paths (considered as edge sets) joining either $R$ and $S$, or $R^{\prime}$ and $S^{\prime}$. Then Seymour's theorem implies a special case of Kleitman, Martin-Löf, Rothschild and Whinston's theorem (see Section 7).

The problem of characterizing similarly the binary clutters with the $\mathbb{Q}_{+}$MFMC-property seems to be hard - see Seymour [1977, 1979 a].

For some related min-max results, see Lovász [1974, 1975, 1976a, 1977], Seymour [1981 b], Schrijver and Seymour [1979].

One may ask for similar results for the anti-blocking case, where we consider the anti-blocker $a(\mathscr{C})$ of $\mathscr{C}$, defined as the collection of all maximal subsets of $S$ intersecting no set in $\mathscr{C}$ in more than one element. In fact the theory here is much more streamlined than in the blocking case, and turns out to reduce completely to perfect graph theory. E. g., if $\mathscr{C}$ is a clutter, and the polytope

$$
\begin{array}{ll}
0 \leqslant x(s) \leqslant 1 & (s \in S), \\
x\left(S^{\prime}\right) \leqslant 1 & \left(S^{\prime} \in \mathscr{C}\right),
\end{array}
$$

has integer vertices (being the incidence vectors of sets in $a(\mathscr{C})$ ), then (16) is totally dual integral, and $\mathscr{E}$ is the collection of all maximal cliques in a perfect graph, while the polytope (16) is just the convex hull of its cocliques - see Fulkerson [1971, 1972], Lovász [1972, 1974], Padberg [1975].

The blocking and the anti-blocking case come together in the so-called balanced matrices, which are $\{0,1\}$-matrices having no square submatrix of odd order with exactly two ones in each row and in each column. Berge [1970, 1972], Berge and Las Vergnas [1970], and Fulkerson, Hoffman and Oppenheim [1974] showed that if $M$ is a balanced matrix, and $w$ is an integer vector, then each of the optima

$$
\begin{aligned}
& \max \{w x \mid x \geqslant 0, M x \leqslant 1\}=\min \{|y| \mid y \geqslant 0, y M \geqslant w\}, \\
& \min \{w x \mid x \geqslant 0, M x \geqslant 1\}=\max \{|y| \mid y \geqslant 0, y M \leqslant w\}
\end{aligned}
$$

have integer optimum solutions. Moreover, the columns of $M$ can be split into $k$ classes, such that each of the classes contains at least one 1 in each of the rows, where $k$ is the minimum number of ones in any row. A similar result holds if we replace "at least" by "at most", and "minimum" by "maximum".

These results can be formulated equivalently in terms of total dual integrality, clutters, blockers, $\mathbb{Z}_{+}$-MFMC-property, anti-blockers, and so on, which we leave to the reader. 
As the incidence matrix of a bipartite graph is balanced, these results extend the results of König, Egerváry, Gupta on bipartite graphs given in Section 4. More generally, each nonnegative totally unimodular matrix is balanced. Another class of balanced matrices are the totally balanced matrices, containing no incidence matrix of a circuit of lenght at least three as a submatrix - see Anstee and Farber [1982], Brouwer and Kolen [1980], Hoffman, Kolen and Sakarovitch [1982], and Lubiw [1982].

\section{Matroids and Submodular Functions}

The concept of matroid, introduced by Whitney [1935] and Van der Waerden [1937] as a framework for graph-theoretic and algebraic studies, turned out to play also a unifying role in combinatorial optimization. This was revealed by the work of Rado [1957] and Edmonds [1970]. In the latter paper also optimization problems for the more general structure of submodular functions were studied.

Here we review briefly the min-max relations for matroids and submodular functions. For a more comprehensive discussion, see Lovász's survey on "Submodular functions and convexity". The standard text on matroid theory is the book of Welsh [1976].

A matroid $M$ is a pair $(S, \mathscr{Y})$, where $S$ is a finite set and $\mathscr{Y}$ is a collection of subsets of $S$ such that

$$
\begin{aligned}
& \text { (i) } \emptyset \in \mathscr{Y}, \\
& \text { (ii) if } S^{\prime \prime} \subseteq S^{\prime} \in \mathscr{Y} \text { then } S^{\prime \prime} \in \mathscr{Y}, \\
& \text { (iii) if } S^{\prime}, S^{\prime \prime} \in \mathscr{Y} \text { and }\left|S^{\prime}\right|<\left|S^{\prime \prime}\right| \text { then } S^{\prime} \cup s \in \mathscr{Y} \text { for some } s \in S^{\prime \prime} \backslash S^{\prime} \text {. }
\end{aligned}
$$

The sets in $\mathscr{Y}$ are called the independent sets of the matroid. The bases are the maximal independent sets. It follows from (1) (iii) that all bases have the same size. Associated with $M$ is its rank function $r: \mathscr{P}(S) \rightarrow \mathbb{Z}$, defined by:

$$
r\left(S^{\prime}\right):=\max \left\{\left|S^{\prime \prime}\right| \mid S^{\prime \prime} \in \mathscr{g}, S^{\prime \prime} \subseteq S^{\prime}\right\}
$$

for $S^{\prime} \subseteq S$. The rank function uniquely determines $M$, as $S^{\prime} \in \mathscr{Y}$ iff $r\left(S^{\prime}\right)=\left|S^{\prime}\right|$.

Examples of matroids are as follows.

I. Graphic matroids (Whitney [1935]). Let $G=(V, E)$ be an undirected graph, and let $\mathscr{Y}$ consist of all subsets $E^{\prime}$ of $E$ not containing any circuit. Then $M=(E, \mathscr{Y})$ is a matroid, with rank function $r$ given by: $r\left(E^{\prime}\right)=|V|-\kappa\left(V, E^{\prime}\right)$, where $\kappa\left(V, E^{\prime}\right)$ denotes the number of components of the graph $\left(V, E^{\prime}\right)$. If $G$ is connected, the bases of $M$ are exactly the spanning trees.

II. Cographic matroids (Whitney [1935]). Let $G=(V, E)$ be an undirected graph, and let $\mathscr{Y}$ consist of all subsets $E^{\prime}$ of $E$ such that the graph $\left(V, E \backslash E^{\prime}\right)$ has the same number of components as $G$ has (i. e., $E^{\prime}$ does not contain a nonempty cut). Then $M=(E, \mathscr{Y})$ is a matroid, with rank func- 
tion $r\left(E^{\prime}\right)=\left|E^{\prime}\right|+\kappa(V, E)-\kappa\left(V, E \backslash E^{\prime}\right)$. If $G$ is connected, the bases of $M$ are exactly the complements of spanning trees.

III. Transversal matroids (Edmonds and Fulkerson [1965]). Let $G=(V, E)$ be a bipartite graph, with colour classes $S$ and $T$, and let $\mathscr{I}$ consist of all subsets $S^{\prime}$ of $S$ for which there exists a matching in $G$ covering $S^{\prime}$. Then $(S, \mathscr{Y})$ is a matroid, with rank function given by $r\left(S^{\prime}\right)=\min _{S^{\prime \prime} \subseteq S^{\prime}}\left|S^{\prime} \backslash S^{\prime \prime}\right|+\left|\Delta\left(S^{\prime \prime}\right)\right|$, where $\Delta\left(S^{\prime \prime}\right)$ denotes the set of vertices adjacent to at least one vertex in $S^{\prime \prime}$ (this min-max relation follows from König's matching theorem (Theorem 2)).

IV. Gammoids (Perfect [1968]). Let $D=(V, A)$ be a directed graph, and let $S$ and $T$ be subsets of $V$. Let $\mathscr{I}$ be the collection of subsets $S^{\prime}$ of $S$ for which there exists $\left|S^{\prime}\right|$ pairwise vertex-disjoint paths starting in $S^{\prime}$ and ending in $T$. Then $(S, \mathscr{Y})$ is a matroid, and its rank function can be written as a min-max relation, using Menger's theorem. Clearly, each transversal matroid is a gammoid.

V. (Linearly) representable matroids (Whitney [1935], Van der Waerden [1937]). Let $S$ be a collection of vectors in a vector space, and let $\mathscr{Y}$ be the collection of linearly independent sets of vectors in $S$. Then $(S, \mathscr{Y})$ is a matroid.

Moreover, Van der Waerden [1937] considered algebraic matroids.

Given a matroid $M=(S, \mathscr{I})$ and a weight function $w: S \rightarrow \mathbb{R}_{+}$, a maximum weighted independet set can be found with the following greedy algorithm: or$\operatorname{der} S=\left\{s_{1}, \ldots, s_{n}\right\}$ such that $w\left(s_{1}\right) \geqslant \ldots \geqslant w\left(s_{n}\right)$, and determine $S_{0}, S_{1}, \ldots, S_{n}$ inductively as follows:

$$
\begin{aligned}
& \text { (i) } S_{0}:=\emptyset \text {, } \\
& \text { (ii) } S_{i+1}:=S_{i} \cup\left\{s_{i+1}\right\}, \quad \text { if } S_{i} \cup\left\{s_{i+1}\right\} \in \mathscr{Y} \text {, } \\
& S_{i+1}:=S_{i}, \quad \text { otherwise, } \\
& \text { for } i=0, \ldots, n-1 \text {. }
\end{aligned}
$$

Then $S_{n}$ is a maximum weighted independent set. Edmonds [1971] and Gale [1968] observed that this characterizes matroids: if $(S, \mathscr{Y})$ satisfies (1) (i) (ii), then $(S, \mathscr{Y})$ is a matroid if and only if the greedy algorithm finds a maximum weighted set in $\mathscr{Y}$, for each weight function.

Edmonds [1970, 1971] derived from the greedy method the following minmax formula: given a matroid $M=(S, \mathscr{Y})$, with rank function $r$, and weight function $w: S \rightarrow \mathbb{Z}_{+}$,

(4) the maximum weight of an independent set is equal to the minimum value of $r\left(S_{1}\right)+\ldots+r\left(S_{k}\right)$, where $S_{1} \subseteq \ldots \subseteq S_{k} \subseteq S$ (repetition allowed), such that each element $s$ of $S$ occurs in at least $w(s)$ of the $S_{i}$.

This is equivalent to the total dual integrality of:

$$
\begin{array}{ll}
x(s) \geqslant 0 & (s \in S), \\
x\left(S^{\prime}\right) \leqslant r\left(S^{\prime}\right) & \left(S^{\prime} \in S\right) .
\end{array}
$$


In particular, (5) determines the matroid polytope of $M$, being the convex hull of the independent sets of $M$. E. g., (4) implies as a special case a good characterization for the maximum (or minimum) length of a spanning tree in a graph. It may be derived from (5) that the convex hull of the forests (i.e., edge sets containing no circuit) in an undirected graph $G=(V, E)$ is determined by:

$$
\begin{array}{ll}
x(e) \geqslant 0 & (e \in E), \\
X\left(\left\langle V^{\prime}\right\rangle\right) \leqslant\left|V^{\prime}\right|-1 & \left(\emptyset \neq V^{\prime} \subseteq V\right)
\end{array}
$$

where $\left\langle V^{\prime}\right\rangle$ denotes the set of edges contained in $V^{\prime}$.

Matroid intersection. Even more interestingly, there is a min-max relation for the maximum weight of a common independent set in two matroids. First consider the cardinality case, for which Edmonds [1970] showed the following.

Theorem 24 (Edmonds' matroid intersection theorem). Let $M_{1}=\left(S, \mathscr{Y}_{1}\right)$ and $M_{2}=\left(S, \mathscr{Y}_{2}\right)$ be matroids, with rank functions $r_{1}$ and $r_{2}$, respectively. Then the maximum size of a set in $\mathscr{Y}_{1} \cap \mathscr{Y}_{2}$ is equal to

$$
\min _{S^{\prime} \subseteq S}\left(r_{1}\left(S^{\prime}\right)+r_{2}\left(S \backslash S^{\prime}\right)\right) .
$$

This generalizes König's matching theorem: if $G=(V, E)$ is a bipartite graph, with colour classes $V_{1}$ and $V_{2}$, let $\mathscr{Y}_{i}=\left\{E^{\prime} \subseteq E \mid\right.$ no two edges in $E^{\prime}$ intersect in $\left.V_{i}\right\}$, for $i=1,2$; then $\left(E, \mathscr{Y}_{1}\right)$ and $\left(E, \mathscr{Y}_{2}\right)$ are matroids, and here Theorem 24 reduces to König's theorem. Applying Theorem 24 to two transversal matroids gives a min-max formula for the maximum size of a common "system of distinct representatives".

Other consequences are the following min-max relations, due to Edmonds [1965 a, 1965 b] and Nash-Williams [1964].

Corollary 24 a (Matroid partition theorems). Let $M=(S, \mathscr{Y})$ be a matroid, with rank function $r$. Then the minimum number of independent sets needed to cover $S$ is equal to $\max _{S^{\prime} \neq \varnothing}\left\lceil\left|S^{\prime}\right| / r\left(S^{\prime}\right)\right\rceil$. Moreover, the maximum number of pairwise disjoint bases is equal to $\min _{S^{\prime} \subseteq S, r\left(S^{\prime}\right) \neq r(S)}\left\lfloor S \backslash S^{\prime} \mid /\left(r(S)-r\left(S^{\prime}\right)\right)\right\rfloor$.

We leave it to the reader to derive this corollary from Theorem 24 (cf. Welsh [1970]).

Specialized to graphic matroids, this corollary yields the following results of Nash-Willams $[1961,1964]$ and Tutte [1961]. Let $G=(V, E)$ be an undirected graph. Then the minimum number of forests needed to cover $E$ is equal to

$$
\max \left\lceil\frac{\left|\left\langle V^{\prime}\right\rangle\right|}{\left|V^{\prime}\right|-1}\right\rceil
$$

where the maximum ranges over all subsets $V^{\prime}$ of $V$ with at least two elements, and where $\left\langle V^{\prime}\right\rangle$ denotes the set of edges contained in $V^{\prime}$. If $G$ is connected, the maximum number of pairwise disjoint spanning trees is equal to

$$
\min \left\lfloor\frac{\left|E \backslash E^{\prime}\right|}{\kappa\left(V, E^{\prime}\right)-1}\right\rfloor
$$


where the minimum ranges over all subsets $E^{\prime}$ of $E$ with $\kappa\left(V, E^{\prime}\right)$ (being the number of components of $\left.\left(V, E^{\prime}\right)\right)$ at least two.

The following weighted version of the matroid intersection theorem was shown also by Edmonds [1970]. Let $M_{1}=\left(S, \mathscr{J}_{1}\right)$ and $M_{2}=\left(S, \mathscr{T}_{2}\right)$ be matroids, with rank functions $r_{1}$ and $r_{2}$, respectively, and let $w: S \rightarrow \mathbb{Z}_{+}$be a weight function. Then:

(10) the maximum weight of a set in $\mathscr{Y}_{1} \cap \mathscr{Y}_{2}$ is equal to the minimum value of $r_{1}\left(S_{1}\right)+\ldots+r_{1}\left(S_{k}\right)+r_{2}\left(T_{1}\right)+\ldots+r_{2}\left(T_{l}\right)$, where $S_{1} \subseteq \ldots \subseteq S_{k} \subseteq S$ and $T_{1} \subseteq \ldots \subseteq T_{l} \subseteq S$ such that each element $s$ of $S$ is contained in at least $w(s)$ of the $S_{1}, \ldots, S_{k}, T_{1}, \ldots, T_{l}$.

This implies the total dual integrality of the system

$$
\begin{array}{ll}
x(s) \geqslant 0 & (s \in S), \\
x\left(S^{\prime}\right) \leqslant r_{1}\left(S^{\prime}\right) & \left(S^{\prime} \subseteq S\right), \\
x\left(S^{\prime}\right) \leqslant r_{2}\left(S^{\prime}\right) & \left(S^{\prime} \subseteq S\right) .
\end{array}
$$

So (11) determines the convex hull of the sets in $\mathscr{Y}_{1} \cap \mathscr{Y}_{2}$. Equivalently, the intersection of two matroid polytopes has integer vertices again. Polynomial algorithms for finding maximum weighted common independent sets were given by Edmonds [1979] and Lawler [1975].

Result (10) contains as special case the weighted version of König's matching theorem. Also Fulkerson's optimum branching theorem (Theorem 16) can be derived.

Submodular functions. In fact, Edmonds [1970] showed more generally the following. A function $f: \mathscr{P}(S) \rightarrow \mathbb{R}$ is called submodular if

$$
f\left(S^{\prime} \cap S^{\prime \prime}\right)+f\left(S^{\prime} \cup S^{\prime \prime}\right) \leqslant f\left(S^{\prime}\right)+f\left(S^{\prime \prime}\right),
$$

for all $S^{\prime}, S^{\prime \prime} \subseteq S$. Now, for submodular $f_{1}, f_{2}: \mathscr{P}(S) \rightarrow \mathbb{R}$, the system

$$
\begin{array}{ll}
x\left(S^{\prime}\right) \leqslant f_{1}\left(S^{\prime}\right) & \left(S^{\prime} \subseteq S\right), \\
x\left(S^{\prime}\right) \leqslant f_{2}\left(S^{\prime}\right) & \left(S^{\prime} \subseteq S\right),
\end{array}
$$

is totally dual integral. Moreover, total dual integrality is maintained if we add $c_{1} \leqslant x \leqslant c_{2}$ to (13), for arbitrary $c_{1}, c_{2}$ (i.e., (13) is box totally dual integral). In particular, if $f_{1}$ and $f_{2}$ (and $c_{1}$ and $c_{2}$ ) are integer-valued, each face of the polyhedron (13) contains integer vectors.

This generalizes the results on matroids given above, as the rank function of any matroid is submodular. Other examples of submodular functions are: (i) let $G=(V, E)$ be a bipartite graph, with colour classes $S$ and $T$, and let $w: T \rightarrow \mathbb{R}_{+}$; then $f\left(S^{\prime}\right):=w\left(\Delta\left(S^{\prime}\right)\right)$ for $S^{\prime} \subseteq S$ is submodular $\left(\Delta\left(S^{\prime}\right)\right.$ denoting the set of vertices in $T$ adjacent to at least one vertex in $\left.S^{\prime}\right)$; (ii) let $D=(V, A)$ be a directed graph, and let $c: A \rightarrow \mathbb{R}_{+}$; then $f\left(V^{\prime}\right):=c\left(\delta^{+}\left(V^{\prime}\right)\right)$ for $V^{\prime} \subseteq V$ is submodular.

Edmonds and Giles [1977] gave a further generalization, containing also graph-theoretical results like the Lucchesi-Younger theorem as special cases. 
Let $\mathscr{C}$ be a family of subsets of the finite set $V$, and let $f: \mathscr{C} \rightarrow \mathbb{R}$ be such that:

(14) if $V^{\prime}, V^{\prime \prime} \in \mathscr{C}$ with $V^{\prime} \cap V^{\prime \prime} \neq \emptyset$ and $V^{\prime} \cup V^{\prime \prime} \neq V$, then $V^{\prime} \cap V^{\prime \prime} \in \mathscr{C}$ and $V^{\prime} \cup V^{\prime \prime} \in \mathscr{C}$, and $f\left(V^{\prime} \cap V^{\prime \prime}\right)+f\left(V^{\prime} \cup V^{\prime \prime}\right) \leqslant f\left(V^{\prime}\right)+f\left(V^{\prime \prime}\right)$.

Then for any directed graph $D=(V, A)$, and $c_{1}, c_{2}: A \rightarrow \mathbb{R} \cup\{ \pm \infty\}$, the system

$$
\begin{array}{ll}
c_{1}(a) \leqslant x(a) \leqslant c_{2}(a) & (a \in A), \\
x\left(\delta^{-}\left(V^{\prime}\right)\right)-x\left(\delta^{+}\left(V^{\prime}\right)\right) \leqslant f\left(V^{\prime}\right) & \left(V^{\prime} \in \mathscr{C}\right),
\end{array}
$$

is totally dual integral. This contains König's matching theorem, the max-flow min-cut theorem, the Lucchesi-Younger theorem, the matroid intersection theorem, Dilworth's theorem, and the total dual integrality of (13) as special cases.

Moreover, if $c_{1} \geqslant 0$, and if for all $V_{1}, V_{2}, V_{3}$ in $\mathscr{C}$ with $V_{1} \subseteq V \backslash V_{2} \subseteq V_{3}$ there is no arc of $D$ entering both $V_{1}$ and $V_{3}$, then the system

$$
\begin{array}{ll}
c_{1}(a) \leqslant x(a) \leqslant c_{2}(a) & (a \in A), \\
x\left(\delta^{-}\left(V^{\prime}\right)\right) \geqslant-f\left(V^{\prime}\right) & \left(V^{\prime} \in \mathscr{C}\right),
\end{array}
$$

is totally dual integral. This contains König's matching theorem, the max-potential min-work theorem, Fulkerson's optimum branching theorem, the Lucchesi-Younger theorem, the matroid intersection theorem, and the total dual integrality of (13) as special cases.

The total dual integrality of (15) and (16) can be shown by extending the method given in Section 8 to prove Fulkerson's optimum branching theorem. Related results, which can be proved similarly, are given in Frank [1979], Grishuhin [1981], Hassin [1978], Hoffman and Schwartz [1978], Lawler and Martel [1982], Schrijver [1982b]. For a survey, see Schrijver [1982 c].

Matroid matching. It is not difficult to see that the following problem generalizes both the matching problem in undirected graphs and the matroid intersection problem: given a matroid $M=(V, \mathscr{Y})$ and an undirected graph $G=(V, E)$, find an independent matching of maximum size. Here an independent matching is a set $E^{\prime}$ of pairwise disjoint edges with $\bigcup E^{\prime}$ independent in $M$.

However, this matroid matching problem (Jenkyns [1974], Lawler [1971, 1976]) is in general NP-hard (B. Korte, and Lovász [1981a]), and a satisfactory min-max formula is likely not to exist.

On the other hand, if $M$ is linearly representable, and given by an explicit representation of $V$ as vectors $v_{1}, \ldots, v_{n}$ in a vector space, Lovász found a polynomial algorithm [1981a], and the following min-max formula [1980a].

Theorem 25. The maximum size of an independent matching is equal to

$$
\min \left(\operatorname{dim} L_{0}+\sum_{i=1}^{t}\left\lfloor\frac{\operatorname{dim} L_{i}-\operatorname{dim} L_{0}}{2}\right\rfloor\right)
$$


which minimum ranges over linear spaces $L_{0}, L_{1}, \ldots, L_{t}(t \geqslant 0)$ such that $L_{0} \subseteq L_{1} \cap \ldots \cap L_{t}$ and such that, for each edge $e$ of $G$, the linear hull of $e$ intersects $L_{0}$ or $e$ is contained in $L_{i}$ for some $i=1, \ldots, t$.

In case $v_{1}, \ldots, v_{n}$ are linearly independent, Theorem 25 reduces to the TutteBerge theorem. Also the matroid intersection theorem for representable matroids is included. Moreover, Mader's theorem on vertex-disjoint $S$-paths (see Section 7) follows as a special case - see Lovász [1980 b], where some more corollaries are described.

It is still an open problem to extend Theorem 25 to the weighted case. Also no polynomial algorithm finding a maximum weighted independent matching has been found as yet. In order to apply the ellipsoid method here, we need a description in terms of linear inequalities of the convex hull of the independent matchings, which is still unknown.

Colourings. Finally we mention the following min-max relations for "supermodular colourings". A collection $\mathscr{C}$ of subsets of the finite set $S$ is called an intersecting family, and the function $g: \mathscr{C} \rightarrow \mathbb{R}$ is called supermodular on intersecting pairs, if the following condition is satisfied:

(18) if $S^{\prime}, S^{\prime \prime} \in \mathscr{C}$ and $S^{\prime} \cap S^{\prime \prime} \neq \emptyset$, then $S^{\prime} \cap S^{\prime \prime} \in \mathscr{C}$ and $S^{\prime} \cup S^{\prime \prime} \in \mathscr{C}$ and $f\left(S^{\prime} \cap S^{\prime \prime}\right)+f\left(S^{\prime} \cup S^{\prime \prime}\right) \geqslant f\left(S^{\prime}\right)+f\left(S^{\prime \prime}\right)$.

Now let $\mathscr{E}_{1}$ and $\mathscr{C}_{2}$ be intersecting families on $S$, and let $g_{1}: \mathscr{C}_{1} \rightarrow \mathbb{Z}$ and $g_{2}: \mathscr{C}_{2} \rightarrow \mathbb{Z}$ be supermodular on intersecting pairs, such that $g_{i}\left(S^{\prime}\right) \leqslant\left|S^{\prime}\right|$ for $i=1,2$ and $S^{\prime} \in \mathscr{C}_{i}$. It may be derived, e.g., from Edmonds and Giles' result on (15) that:

$$
\begin{aligned}
& \min \left\{\left|S^{\prime \prime}\right|\left|S^{\prime \prime} \subseteq S,\right| S^{\prime \prime} \cap S^{\prime} \mid \geqslant g_{i}\left(S^{\prime}\right) \text { for all } i=1,2 \text { and } S^{\prime} \in \mathscr{C}_{i}\right\} \\
& =\max \left\{g_{1}\left(S_{1}\right)+\ldots+g_{1}\left(S_{m}\right)+g_{2}\left(T_{1}\right)+\ldots+g_{2}\left(T_{l}\right) \mid S_{1}, \ldots, S_{m} \in \mathscr{C}_{1},\right. \\
& \left.T_{1}, \ldots, T_{l} \in \mathscr{C}_{2}, \text { and } S_{1}, \ldots, S_{m}, T_{1}, \ldots, T_{l} \text { pairwise disjoint }\right\}
\end{aligned}
$$

This can be considered as a theorem on the minimum size of a common "spanning set" in two matroids. Now also the following, more or less "polar", minmax result holds (cf. Schrijver [1983 b]):

(20) $\min \left\{k \mid\right.$ there exist pairwise disjoint subsets $S_{1}, \ldots, S_{k}$ of $S$ such that, for all $i=1,2$ and $S^{\prime} \in \mathscr{C}_{i}, \quad S^{\prime}$ intersects at least $g_{i}\left(S^{\prime}\right)$ of the $\left.S_{i}\right\}=\max \left\{g_{i}\left(S^{\prime}\right) \mid i=1,2 ; S^{\prime} \in \mathscr{C}_{i}\right\}$

(assuming $g_{i}\left(S^{\prime}\right) \geqslant 0$ for at least one $i$ and $\left.S^{\prime}\right)$. This relation implies König's and Gupta's edge-colouring theorems (Theorem 3 and Corollary 3 a).

\section{References}

[1982] R. P. Anstee and M. Farber, Characterizations of totally balanced matrices, Research Report CORR 82-5, Faculty of Mathematics, University of Waterloo, Waterloo, Ont., 1982.

[1981] E. Balas and N. Christofides, A restricted Lagrangean approach to the traveling salesman problem, Math. Programming 21 (1981) 19-46. 
[1950] H. B. Belck, Reguläre Faktoren von Graphen, J. Reine Angew. Math. 188 (1950) 228-252.

[1958] C. Berge, Sur le couplage maximum d'un graphe, C. R. Acad. Sci. Paris 247 (1958) 258-259.

[1960] C. Berge, Les problèmes de coloration en théorie des graphes, Publ. Inst. Stat. Univ. Paris 9 (1960) 123-160.

[1961] C. Berge, Farbung von Graphen deren sämtliche bzw. ungerade Kreise starr sind (Zusammenfassung), Wiss. Z. Martin-Luther-Univ. Halle-Wittenberg, Math.-Natur. Reihe (1961) 114-115.

[1962] C. Berge, Sur un conjecture relative au problème des codes optimaux, Commun. 13ème Assemblée Gén. U.R.S.I., Tokyo, 1962.

[1969] C. Berge, The rank of a family of sets and some applications to graph theory, in: Recent progress in combinatorics (W. T. Tutte, ed.), Acad. Press, New York, 1969, pp. 246-257.

[1970] C. Berge, Sur certain hypergraphes generalisant les graphes bipartis, in: Combinatorial theory and its applications (P. Erdös, A. Rényi, and V. T. Sós, eds.), North-Holland, Amsterdam, 1970, pp. 119-133.

[1972] C. Berge, Balanced matrices, Math. Programming 2 (1972) 19-31.

[1982] C. Berge and V. Chvátal (eds.), Perfect graphs, to appear.

[1970] C. Berge and M. Las Vergnas, Sur un théorème du type König pour hypergraphes, in: Proc. Intern. Conf. on Comb. Math. (A. Gewirtz and L. Quintas, eds.), Ann. New York Acad. Sci. 175 (1970) 32-40.

[1946] G. Birkhoff, Tres observaciones sobre el algebra lineal, Rev. Univ. Nac. Tucuman Ser. A 5 (1946) 147-148.

[1976] J. A. Bondy and U. S. R. Murty, Graph theory with applications, Macmillan, London, 1976.

[1980] A. E. Brouwer and A. Kolen, A super-balanced hypergraph has a nest point, Report ZW 148/80, Math. Centrum, Amsterdam, 1980.

[1982] K. Cameron, Polyhedral and algorithmic ramifications of antichains, Ph. D. thesis, University of Waterloo, Waterloo, Ont., 1982.

[1975] V. Chvátal, On certain polytopes associated with graphs, J. Combinatorial Theory (B) 18 (1975) 138-154.

[1981] V. Chvátal, communication C.I.R.M. Marseille-Luminy, 1981.

[1980] G. Cornuéjols and W. R. Pulleyblank, A matching problem with side conditions, Discrete Math. 29 (1980) 135-159.

[1983] W. Cook and W. R. Pulleyblank, to appear.

[1978] W. H. Cunningham and A. B. Marsh, A primal algorithm for optimal matching, Math. Programming Study 8 (1978) 50-72.

[1951] G. B. Dantzig, Application of the simplex method to a transportation problem, in: Activity analysis of production and allocation (T. C. Koopmans, ed.), J. Wiley, New York, 1951, pp. 359-373.

[1979] R. W. Deming, Independence numbers of graphs - an extension of the KönigEgerváry theorem, Discrete Math. 27 (1979) 23-33.

[1971] M. A. H. Dempster, Two algorithms for the time-table problem, in: Combinatorial mathematics and its applications (D. J. A. Welsh, ed.), Acad. Press, New York, 1971, pp. 63-85.

[1950] R. P. Dilworth, A decomposition theorem for partially ordered sets, Ann. of Math. 51 (1950) 161-166.

[1961] G. A. Dirac, On rigid circuit graphs, Abh. Math. Sem. Univ. Hamburg 25 (1961) $71-76$. 
[1965 a] J. Edmonds, Minimum partition of a matroid into independent subsets, J. Res. Nat. Bur. Standards Sect. B 69 (1965) 67-72.

[1965 b] J. Edmonds, Lehman's switching game and a theorem of Tutte and Nash-Williams, J. Res. Nat. Bur. Standards Sect. B 69 (1965) 73-77.

[1965 c] J. Edmonds, Paths, trees, and flowers, Canad. J. Math. 17 (1965) 449-467.

[1965 d] J. Edmonds, Maximum matching and a polyhedron with 0,1-vertices, J. Res. Nat. Bur. Standards Sect. B69 (1965) 125-130.

[1967] J. Edmonds, An introduction to matching, mimeographed notes, Engineering Summer Conf., Univ. of Michigan, Ann Arbor, 1967.

[1967 a] J. Edmonds, Optimum branchings, J. Res. Nat. Bur. Standards Sect. B 71 (1967) 233-240.

[1970] J. Edmonds, Submodular functions, matroids, and certain polyhedra, in: Combinatorial structures and their applications (R. Guy, H. Hanani, N. Sauer and J. Schönheim, eds.), Gordon and Breach, New York, 1970, pp. 69-87.

[1971] J. Edmonds, Matroids and the greedy algorithm, Math. Programming 1 (1971) $127-136$.

[1973] J. Edmonds, Edge-disjoint branchings, in: Combinatorial algorithms (B. Rustin, ed.), Acad. Press, New York, 1973, pp. 91-96.

[1979] J. Edmonds, Matroid intersection, Annals of Discrete Math. 4 (1979) 39-49.

[1965] J. Edmonds and D. R. Fulkerson, Transversals and matroid partition, J. Res. Nat. Bur. Standards Sect. B 69 (1965) 147-153.

[1970] J. Edmonds and D. R. Fulkerson, Bottleneck extrema, J. Combinatorial Theory 8 (1970) 299-306.

[1977] J. Edmonds and R. Giles, A min-max relation for submodular functions on graphs, Annals of Discrete Math. 1 (1977) 185-204.

[1970] J. Edmonds and E. L. Johnson, Matching, a well-solved class of integer linear programs, in: Combinatorial structures and their applications (R. Guy, H. Hanani, N. Sauer and J. Schönheim, eds.), Gordon and Breach, New York, 1970, pp. 89-92.

[1973] J. Edmonds and E. L. Johnson, Matching, Euler tours and the Chinese postman, Math. Programming 5 (1973) 88-124.

[1972] J. Edmonds and R. M. Karp, Theoretical improvements in algorithmic efficiency for network flow problems, J. ACM 19 (1972) 248-264.

[1931] E. Egerváry, Matrixok kombinatorius tulajdonságairol, Mat. Fiz. Lapok 38 (1931) 16-28.

[1956] P. Elias, A. Feinstein and C. E. Shannon, A note on the maximum flow through a network, IRE Trans. Information Theory IT 2 (1956) 117-119.

[1956] L. R. Ford and D. R. Fulkerson, Maximum flow through a network, Canad. J. Math. 8 (1956) 399-404.

[1958] L. R. Ford and D. R. Fulkerson, Network flow and systems of distinct representatives, Canad. J. Math. 10 (1958) 78-84.

[1962] L. R. Ford and D. R. Fulkerson, Flows in networks, Princeton Univ. Press, Princeton, N.J., 1962.

[1979] A. Frank, Kernel systems of directed graphs, Acta Sci. Math. (Szeged) 41 (1979) 63-76.

[1980] A. Frank, On chain and antichain families of a partially ordered set, J. Combinatorial Theory (B) 29 (1980) 176-184.

[1981] A. Frank, How to make a digraph strongly connected, Combinatorica 1 (1981) 145-153.

[1912] G. Frobenius, Über Matrizen aus nicht negativen Elementen, Sitzber. Preuss. Akad. Wiss. (1912) 456-477. 
[1917] G. Frobenius, Über zerlegbare Determinanten, Sitzber. Preuss. Akad. Wiss. (1917) 274-277.

[1956] D. R. Fulkerson, Note on Dilworth's decomposition theorem for partially ordered sets, Proc. Amer. Math. Soc. 7 (1956) 701-702.

[1961] D. R. Fulkerson, An out-of-kilter method for minimal cost flow problems, SIAM J. Appl. Math. 9 (1961) 18-27.

[1968] D. R. Fulkerson, Networks, frames, and blocking systems, in: Mathematics of the decision sciences, part I (G. B. Dantzig and A. F. Veinott, eds.), Amer. Math. Soc., Providence, R. I., 1968, pp. 303-334.

[1970] D. R. Fulkerson, Blocking polyhedra, in: Graph theory and its applications (B. Harris, ed.), Acad. Press, New York, 1970, pp. 93-112.

[1971] D. R. Fulkerson, Blocking and anti-blocking pairs of polyhedra, Math. Programming 1 (1971) 168-194.

[1972] D. R. Fulkerson, Anti-blocking polyhedra, J. Combinatorial Theory (B) 12 (1972) 50-71.

[1974] D. R. Fulkerson, Packing rooted directed cuts in a weighted directed graph, Math. Programming 6 (1974) 1-13.

[1974] D. R. Fulkerson, A. J. Hoffman and R. Oppenheim, On balanced matrices, Math. Programming Study 1 (1974) 120-132.

[1968] D. Gale, Optimal assignments in an ordered set: an application of matroid theory, J. Combinatorial Theory 4 (1968) 176-180.

[1958] T. Gallai, Maximum-minimum Sätze über Graphen, Acta Math. Acad. Sci. Hungar. 9 (1958) 395-434.

[1959] T. Gallai, Über extreme Punkt- und Kantenmengen, Ann. Univ. Sci. Budapest, Eötvos Sect. Math. 2 (1959) 133-138.

[1979] M. R. Garey and D. S. Johnson, Computers and intractability: a guide to the theory of NP-completeness, Freeman, San Francisco, 1979.

[1973] F. Gavril, Algorithms for a maximum clique and a maximum independent set of a circle graph, Networks 3 (1973) 261-273.

[1974] F. Gavril, Algorithms on circular-arc graphs, Networks 4 (1974) 357-369.

[1962] A. Ghouila-Houri, Caractérisation des matrices totalement unimodulaires, C. R. Acad. Sci. Paris 254 (1962) 1192-1194.

[1982 a] R. Giles, Optimum matching forests I: special weights, Math. Programming 22 (1982) 1-11.

[1982 b] R. Giles, Optimum matching forests II: general weights, Math. Programming 22 (1982) 12-38.

[1982 c] R. Giles, Optimum matching forests III: facets of matching forest polyhedra, Math. Programming 22 (1982) 39-51.

[1981] R. Giles and L. E. Trotter, On stable set polyhedra for $K_{1,9}$ free graphs, J. Combinatorial Theory (B) 31 (1981) 313-326.

[1980] M. C. Golumbic, Algorithmic graph theory and perfect graphs, Acad. Press, New York, 1980.

[1976] C. Greene, Some partitions associated with a partially ordered set, J. Combinatorial Theory (A) 20 (1976) 69-79.

[1976] C. Greene and D. J. Kleitman, The structure of Sperner $k$-families, J. Combinatorial Theory (A) 20 (1976) 41-68.

[1981] V. P. Grishuhin, Polyhedra related to a lattice, Math. Programming 21 (1981) 70-89.

[1981] M. Grötschel, L. Lovász and A. Schrijver, The ellipsoid method and its consequences in combinatorial optimization, Combinatorica 1 (1981) 169-197. 
[1981 a] M. Grötschel, L. Lovász and A. Schrijver, Polynomial algorithms for perfect graphs, Res. Report WP 81.176-OR, Inst. Oper. Research, Univ. Bonn, 1981.

[1967] R. P. Gupta, A decomposition theorem for bipartite graphs, in: Theory of graphs (P. Rosenstiehl, ed.), Gordon and Breach, New York, 1967, pp. 135138.

[1978] R. P. Gupta, An edge-colouring theorem for bipartite graphs with applications, Discrete Math. 23 (1978) 229-233.

[1981] E. Györi, A minimax theorem on intervals, preprint Math. Inst. Hung. Acad. Sci. No. 54/1981, Budapest, 1981.

[1958] A. Hajnal and J. Surányi, Über die Auflösung von Graphen in vollständigen Teilgraphen, Ann. Univ. Sci. Budapest, Eötvös Sect. Math. 1 (1958) 113-121.

[1935] P. Hall, On representatives of subsets, J. London Math. Soc. 10 (1935) 26-30.

[1978] R. Hassin, On network flows, Ph. D. thesis, Yale Univ., Boston, 1978.

[1960] A. J. Hoffman, Some recent applications of the theory of linear inequalities to extremal combinatorial analysis, in: Combinatorial analysis (R. E. Bellman and M. Hall, eds.), Amer. Math. Soc., Providence, R. I., 1960, pp. 113-127.

[1982] A. J. Hoffman, A. W. J. Kolen and M. Sakarovitch, Totally-balanced and greedy matrices, Report BW, Math. Centrum, Amsterdam, 1982.

[1956] A. J. Hoffman and J. B. Kruskal, Integral boundary points of convex polyhedra, in: Linear inequalities and related systems $(H$. W. Kuhn and A. W. Tucker, eds.), Ann. of Math. Studies 38, Princeton Univ. Press, Princeton, N. J., 1956, pp. 233-246.

[1963] A. J. Hoffman and H. M. Markowitz, A note on shortest path, assignment, and transportation problems, Naval Res. Logist. Quart. 10 (1963) 375-380.

[1977] A. J. Hoffman and D. E. Schwartz, On partitions of partially ordered sets, J. Combinatorial Theory (B) 23 (1977) 3-13.

[1978] A. J. Hoffman and D. E. Schwartz, On lattice polyhedra, in: Combinatorics (A. Hajnal and V. T. Sós, eds.), North-Holland, Amsterdam, 1978, pp. 593-598.

[1981] W.-L. Hsu, Y. Ikura and G. L. Nemhauser, A polynomial algorithm for maximum weighted vertex packings on graphs without long odd cycles, Math. Programming 20 (1981) 225-232.

[1963] T. C. Hu, Multicommodity network flows, Operations Res. 11 (1963) 344 360.

[1973] T. C. Hu, Two-commodity cut packing problem, Discrete Math. 4 (1973) 108 109.

[1974] T. A. Jenkyns, Matchoids: a generalization of matchings and matroids, Ph. D. thesis, Univ. of Waterloo, Waterloo, Ont., 1974.

[1979] A. V. Karzanov, On the minimal number of arcs of a digraph meeting all its directed cutsets, to appear.

[1970] D. J. Kleitman, A. Martin-Löf, B. Rothschild and A. Whinston, A matching theorem for graphs, J. Combinatorial Theory 8 (1970) 104-114.

[1915] D. König, Vonalrendszerek és determinánsok (Line-systems and determinants), Matematikai és Természettudományi Értesítö 33 (1915) 221-229 (in Hungarian).

[1916] D. König, Graphen und ihre Anwendung auf Determinantentheorie und Mengenlehre, Math. Ann. 77 (1916) 453-465.

[1931] D. König, Graphok és matrixok, Mat. Fiz. Lapok 38 (1931) 116-119.

[1932] D. König, Über trennende Knotenpunkte in Graphen (nebst Anwendungen auf Determinanten und Matrizen), Acta. Lit. Sci. Sect. Sci. Math. (Szeged) 6 (19321934) $155-179$.

[1955] H. W. Kuhn, The Hungarian method for solving the assignment problem, Naval Res. Logist. Quart. 2 (1955) 83-97. 
[1956] H. W. Kuhn, Variants of the Hungarian method for the assignment problem, Naval Res. Logist. Quart. 3 (1956) 253-258.

[1971] E. L. Lawler, Matroids with parity conditions: a new class of combinatorial optimization problems, Memorandum ERL-M334, Univ. of California, Berkeley, 1971.

[1975] E. L. Lawler, Matroid intersection algorithms, Math. Programming 9 (1975) 3156.

[1976] E. L. Lawler, Combinatorial optimization: networks and matroids, Holt, Rinehart and Winston, New York, 1976.

[1982] E. L. Lawler and C. U. Martel, Computing maximal "polymatroidal" network flows, Math. of Oper. Research 7 (1982) 334-347.

[1979] A. Lehman, On the width-length inequality, Math. Programming 16 (1979) 245259.

[1978] M. V. Lomonosov, On the systems of flows in a network, Probl. Per. Inf. 14 (1978) 60-73 (in Russian); English translation: Problems of Inf. Transmission 14 (1978) 280-290.

[1982] M. V. Lomonosov, Combinatorial approach to multi-flow problems, preprint.

[1970] L. Lovász, Subgraphs with prescribed valencies, J. Combinatorial Theory 8 (1970) 391-416.

[1972] L. Lovász, Normal hypergraphs and the perfect graph conjecture, Discrete Math. 2 (1972) 253-267.

[1974] L. Lovász, Minimax theorems for hypergraphs, in: Hypergraph seminar (C. Berge and D. Ray-Chaudhuri, eds.), Springer Lecture Notes in Mathematics 411, Springer, Berlin, 1974, pp. 111-126.

[1975] L. Lovász, 2-Matchings and 2-covers of hypergraphs, Acta. Math. Acad. Sci. Hungar. 26 (1975) 433-444.

[1976 a] L. Lovász, On two minimax theorems in graph theory, J. Combinatorial Theory (B) 21 (1976) 96-103.

[1976b] L. Lovász, On some connectivity properties of Eulerian graphs, Acta. Math. Acad. Sci. Hungar. 28 (1976) 129-138.

[1977] L. Lovász, Certain duality principles in integer programming, Annals of Discrete Math. 1 (1977) 363-374.

[1979] L. Lovász, On the Shannon capacity of a graph, IEEE Trans. Inform. Theory IT 25 (1979) 1-7.

[1979 a] L. Lovász, Graph theory and integer programming, Annals of Discrete Math. 4 (1979) 141-158.

[1980 a] L. Lovász, Selecting independent lines from a family of lines in a space, Acta Sci. Math. (Szeged) 42 (1980) 121-131.

[1980b] L. Lovász, Matroid matching and some applications, J. Combinatorial Theory (B) 28 (1980) 208-236.

[1981 a] L. Lovász, The matroid matching problem, in: Algebraic methods in graph theory (L. Lovász and V. T. Sós, eds.), North-Holland, Amsterdam, 1981, pp. 495517.

[1981 b] L. Lovász, Perfect graphs, in: More selected topics in graph theory (L. W. Beineke and R. J. Wilson, eds.), to appear.

[1982] L. Lovász, Ear-decompositions of matching-covered graphs, preprint, 1982.

[1982] A. Lubiw, $\Gamma$-free matrices, M. Sc. thesis, Univ. of Waterloo, Waterloo, Ont., 1982.

[1976] C. L. Lucchesi, A minimax equality for directed graphs, Ph. D. thesis, Univ. of Waterloo, Waterloo, Ont., 1976.

[1978] C. L. Lucchesi and D. H. Younger, A minimax relation for directed graphs, J. London Math. Soc. (2) 17 (1978) 369-374. 
[1978 a] W. Mader, Über die Maximalzahl kantendisjunkter A-Wege, Arch. Math. (Basel) 30 (1978) 325-336.

[1978b] W. Mader, Über die Maximalzahl kreuzungsfreier H-Wege, Arch. Math. (Basel) 31 (1978) 387-402.

[1979] A. B. Marsh, Matching algorithms, Ph. D. thesis, Johns Hopkins Univ., Baltimore, 1979.

[1972] C. J. H. McDiarmid, The solution of a time-tabling problem, J. Inst. Maths. Appl. 9 (1972) 23-34.

[1927] K. Menger, Zur allgemeinen Kurventheorie, Fund. Math. 10 (1927) 96-115.

[1976] H. Meyniel, On the perfect graph conjecture, Discrete Math. 16 (1976) 339342.

[1960] G. J. Minty, Monotone networks, Proc. Roy. Soc. London Ser. A 257 (1960) 194-212.

[1980] G. J. Minty, On maximal independent sets of vertices in a claw-free graph, J. Combinatorial Theory (B) 28 (1980) 284-304.

[1971] L. Mirsky, Transversal theory, Acad. Press, London, 1971.

[1961] C. St. J. A. Nash-Williams, Edge-disjoint spanning trees of finite graphs, J. London Math. Soc. 36 (1961) 445-450.

[1964] C. St. J. A. Nash-Williams, Decomposition of finite graphs into forests, J. London Math. Soc. 39 (1964) 12.

[1953] J. von Neumann, A certain zero-sum two-person game equivalent to the optimum assignment problem, in: Contributions to the theory of games II (A. W. Tucker and H. W. Kuhn, eds.), Annals of Math. Studies 38, Princeton Univ. Press, Princeton, N.J., 1953, pp. 5-12.

[1981] H. Okamura and P. D. Seymour, Multicommodity flows in planar graphs, J. Combinatorial Theory (B) 31 (1981) 75-81.

[1956] A. Orden, The transshipment problem, Manag. Sci. 2 (1956) 276-285.

[1975] M. Padberg, Characterisation of totally unimodular, balanced and perfect matrices, in: Combinatorial programming: methods and applications (B. Roy, ed.), Reidel, Dordrecht (Holland), 1975, pp. 275-284.

[1982] M. W. Padberg and M. R. Rao, Odd minimum cut-sets and b-matchings, Math. of Oper. Res. 7 (1982) 67-80.

[1982] C. H. Papadimitriou and K. Steiglitz, Combinatorial optimization: algorithms and complexity, Prentice-Hall, Englewood Cliffs, N.J., 1982.

[1976] B. A. Papernov, Feasibility of multicommodity flows, in: Studies in Discrete Optimization (A. A. Fridman, ed.), Izdat. "Nauka", Moscow, 1976, pp. 230-261 (in Russian).

[1968] H. Perfect, Applications of Menger's graph theorem, J. Math. Analysis Appl. 22 (1968) 96-111.

[1973] W. R. Pulleyblank, Faces of matching polyhedra, Ph. D. thesis, Univ. of Waterloo, Waterloo, Ont., 1973.

[1980] W. R. Pulleyblank, Dual integrality in b-matching problems, Math. Programming Study 12 (1980) 176-196.

[1983] W. R. Pulleyblank, Polyhedral combinatorics, this volume.

[1957] R. Rado, A note on independence functions, Proc. London Math. Soc. 7 (1957) 300-320.

[1966 a] B. Rothschild and A. Whinston, On two-commodity network flows, Operations Res. 14 (1966) 377-387.

[1966 b] B. Rothschild and A. Whinston, Feasibility of two-commodity network flows, Operations Res. 14 (1966) 1121-1129.

[1978] N. Sbihi, Étude des stables dans les graphes sans étoile, M. Sc. thesis, Univ. Sci. et Méd. Grenoble, 1978. 
[1981] N. Sbihi and J. P. Uhry, A class of h-perfect graphs, Rapport de Rech. No. 236, IRMA, Grenoble, 1981.

[1980] A. Schrijver, A counterexemple to a conjecture of Edmonds and Giles, Discrete Math. 32 (1980) 213-214.

[1981] A. Schrijver, Short proofs on the matching polyhedron, Rapport AE 17/81, Inst. Act. \& Econ., Univ. van Amsterdam, Amsterdam, 1981 (J. Combinatorial Theory (B), to appear).

[1982 a] A. Schrijver, Min-max relations for directed graphs, Annals of Discrete Math. 16 (1982) 261-280.

[1982 b] A. Schrijver, Proving total dual integrality with cross-free families - a general framework, Report AE 5/82, Inst. Act. \& Econ., Univ. van Amsterdam, Amsterdam, 1982 (Math. Programming, to appear).

[1982 c] A. Schrijver, Total dual integrality from directed graphs, crossing families, and sub- and supermodular functions, Proc. Waterloo 1982, to appear.

[1983 a] A. Schrijver, Packing and covering of crossing families of cuts, Report AE 1/ 83, Univ. van Amsterdam, Amsterdam, 1983.

[1983 b] A. Schrijver, Supermodular colourings, Report AE 4/83, Univ. van Amsterdam, Amsterdam, 1983.

[1977] A. Schrijver and P. D. Seymour, A proof of total dual integrality of matching polyhedra, Report ZN 79/77, Math. Centrum, Amsterdam, 1977.

[1979] A. Schrijver and P. D. Seymour, Solution of two fractional packing problems of Lovász, Discrete Math. 26 (1979) 177-184.

[1977] P. D. Seymour, The matroids with the max-flow min-cut property, J. Combinatorial Theory (B) 23 (1977) 189-222.

[1978 a] P. D. Seymour, A two-commodity cut theorem, Discrete Math. 23 (1978) 177181.

[1978 b] P. D. Seymour, Sums of circuits, in: Graph theory and related topics (J. A. Bondy and U. S. R. Murty, eds.), Acad. Press, New York, 1978, pp. 341-355.

[1979 a] P. D. Seymour, On multi-colourings of cubic graphs, and conjectures of Fulkerson and Tutte, Proc. London Math. Soc. (3) 38 (1979) 423-460.

[1979b] P. D. Seymour, A short proof of the two-commodity flow theorem, J. Combinatorial Theory (B) 26 (1979) 370-371.

[1980] P. D. Seymour, Four-terminus flows, Networks 10 (1980) 79-86.

[1981 a] P. D. Seymour, On odd cuts and plane multicommodity flows, Proc. London Math. Soc. (3) 42 (1981) 178-192.

[1981 b] P. D. Seymour, Matroids and multicommodity flows, Europ. J. Comb. 2 (1981) 257-290.

[1979] F. Sterboul, A characterization of the graphs in which the transversal number equals the matching number, J. Combinatorial Theory (B) 27 (1979) 228-229.

[1970] J. Stoer and C. Witzgall, Convexity and optimization in finite dimensions I, Springer, Berlin, 1970.

[1947] W. T. Tutte, The factorization of linear graphs, J. London Math. Soc. 22 (1947) $107-111$.

[1952] W. T. Tutte, The factors of graphs, Canad. J. Math. 4 (1952) 314-328.

[1953] W. T. Tutte, The 1-factors of oriented graphs, Proc. Amer. Math. Soc. 4 (1953) 922-931.

[1954] W. T. Tutte, A short proof of the factor theorem for finite graphs, Canad. J. Math. 6 (1954) 347-352.

[1961] W. T. Tutte, On the problem of decomposing a graph into $n$ connected factors, J. London Math. Soc. 36 (1961) 221-230.

[1981] W. T. Tutte, Graph factors, Combinatorica 1 (1981) 79-97. 
[1964] V. G. Vizing, On an estimate of the chromatic class of a p-graph, Diskret. Analiz. 3 (1964) 25-30 (in Russian).

[1937] B. L. van der Waerden, Moderne Algebra, Springer, Berlin, 1937.

[1970] D. J. A. Welsh, On matroid theorems of Edmonds and Rado, J. London Math. Soc. 2 (1970) 251-256.

[1976] D. J. A. Welsh, Matroid theory, Acad. Press, London, 1976.

[1970] D. de Werra, On some combinatorial problems arising in scheduling, Canad. Oper. Res. Soc. J. 8 (1970) 165-175.

[1972] D. de Werra, Decomposition of bipartite multigraphs into matchings, Zeitschr. Oper. Res. 16 (1972) 85-90.

[1935] $H$. Whitney, On the abstract properties of linear independence, Amer. J. Math. 57 (1935) 509-533.

[1972] R. J. Wilson, Introduction to graph theory, Oliver and Boyd, Edinburgh, 1972.

Acknowledgements. I thank Dr. W. Cook and Dr. W. R. Pulleyblank for their helpful comments. 